\title{
Tutorial on Fedback Control of Flows, Part I: Stabilization of Fluid Flows in Channels and Pipes*
}

\author{
OLE MORTEN AAMO and THOR I. FOSSEN†
}

Keywords: Flow control, stabilization, feedback

\begin{abstract}
The field of flow control has picked up pace over the past decade or so, on the promise of real-time distributed control on turbulent scales being realizable in the near future. This promise is due to the micromachining technology that emerged in the 1980s, and developed at an amazing speed through the 1990s. In lab experiments, so called micro-electro-mechanical systems (MEMS) that incorporate the entire detection-decision-actuation process on a single chip, have been batch processed in large numbers and assembled into flexible skins for gluing onto bodyfluid interfaces for drag reduction purposes. Control of fluid flows span a wide variety of specialities. In Part I of this tutorial, we focus on the problem of reducing drag in channel and pipe flows by stabilizing the parabolic equilibrium profile using boundary feedback control. The control strategies used for this problem include classical control, based on the Nyquist criteria, and various optimal control techniques $\left(\mathscr{H}_{2}, \mathscr{H}_{\infty}\right)$, as well as applications of Lyapunov stability theory.
\end{abstract}

\section{Introduction}

\subsection{Why flow control?}

Flow control involves controlling a flow field using passive or active devices in order to bring on desired changes in the behavior of the flow. For instance, laminar flow, which is characterized by paralel layers of fluid moving in a very regular and deterministic way, is associated with considerable less drag, or friction, at wall-fluid interfaces, than its counterpart, turbulent flow, which is characterized by small scale velocity components that appear to be stochastic in nature. On the other hand, turbulent flow may exhibit better mixing properties than laminar flows. Usually, laminar flows are unstable, and will unless controlled, evolve into turbulent flows. Common control objectives include (Gad-el-Hak, 2000):

- Delaying or advancing transition from laminar to turbulent flow;

- Suppressing or enhancing turbulence, and;

- Preventing or provoking separation.

The benefits that can be gained from these control objectives include drag reduction, lift enhancement, mixing enhancement, and flow-induced noise suppression. For example, a turbulent pipe flow induces considerable drag, or friction, at the bounding wall. It is the resulting overall drag force that the compressor has to overcome in order to pump fluid through the pipe. Increasing the throughput can be achieved by simply installing a more powerful compressor, but the result would be

*Submitted to MIC on 3 January, 2002.

$\uparrow$ Department of Engineering Cybernetics, Norwegian University of Science and Technology, N-7491 Trondheim, Norway. 
increased energy consumption. Since laminar flow induces much less drag, designing a flow control system that relaminarizes the flow will permit higher throughput without increasing energy consumption.

Mixing processes are encountered frequently in applications, and the quality of the resulting mixture directly affects the quality of the end product. This is the case, for instance, in combustion, where the quality of the fuel-air mixture is essential for power generation, and in process industry, where the quality of various mixtures affect chemical reaction rates and the purity of end products. Mixing is usually obtained using 'brute-force' techniques, such as mechanical stirring, jet injection and stirring valves. These methods, and all other methods for mixing, are associated with a drag penalty. The application of flow control to mixing problems seeks to minimize this penalty.

In flows past bluff bodies, vortex shedding occurs. Vortices are periodically shed, alternating between the upper and lower part of the body, leading to periodic forcing of the body. This may be a problem, for instance for offshore structures, because the body starts to vibrate which eventually leads to destruction from fatigue. By applying flow control, one may alter the behaviour of the flow around the structure in such a manner that vortex shedding is suppressed or dampened, prolonging the lifetime of the structure.

The boundary feedback laws designed for these problems are distributed. That is; sensing and actuation are applied at every point on the boundary of the flow domain. While this may sound unrealistic, the micromachining technology that emerged in the 1980 s, permits rapid sensing and actuation on the micron scale, and thereby enables real-time distributed control of fluid flows.

It is clear from the examples mentioned above that the main objective in flow control is to lower operational expenses. We conclude this motivational section with a quote from Gad-el-Hak (2000): 'The potential benefits of realizing efficient flowcontrol systems range from saving billions of dollars in annual fuel costs for land, air, and sea vehicles to achieving economically and environmentally more competitive industrial processes involving fluid flows.'

\subsection{Scope of this tutorial}

The concept of flow control contains a wide variety of theoretical and technological branches, as the examples in the previous section suggest. In this tutorial we will narrow the treatment down to recent developments in two problem areas that have attracted much attention. The first is stabilization of internal prototype flows, namely the channel flow (2D or 3D) and the pipe flow. The channel flow is the flow contained between two paralel plates, and the pipe flow is the flow contained in the interior of a cylinder with circular cross section. The second problem we will study, is mixing in flows. Mixing is the topic of Part II of this tutorial.

Stabilization of incompressible fluid flow in a plane channel has been studied quite extensively, and the wall sensing/actuation of this flow has become a standard benchmark problem in the area of flow control, see, e.g., Bewley (2001) and Gad-elHak (1996) for recent reviews. In Joshi et al. (1997) stabilizing PI controllers for twodimensional channel flow were designed for a reduced-order model of the linearized Navier-Stokes equation, obtained by a standard Galerkin procedure. The work was continued in Joshi et al. (1999), where LQG design was applied in order to obtain optimal controllers for this reduced-order model. LQG/LTR of the streamfunction 
formulation of the Navier-Stokes equation was also the focus of Cortelezzi et al. (1998) and Cortelezzi and Speyer (1998), where the latter reference reports the remarkable result of drag reduction to $50 \%$ below the laminar level. In Kang et al. (1999), a reduced-order model of 3D perturbations (at a single wavenumber pair $\left.\left\{k_{x}, k_{z}\right\}\right)$ was developed, and LQG control design was applied to this model. Drag reduction by means of body forcing inside the domain applicd through electromagnetic forcing was suggested in Baker et al. (2000), where an observer-based approach was applied to a reduced order, linearized model. A nonlinear attempt was presented in Christofides and Armaou (1998), where Galerkin's method was used to derive a reduced-order model of the full, nonlinear, two-dimensional Navier-Stokes equation. A nonlinear control law was given, along with conditions under which closed-loop stability is obtained. The results were applied to Burger's equation, which is the one-dimensional Navier-Stokes equation, including the nonlinear advective term. In Bewley and Liu (1998), LQG and $\mathscr{H}_{\infty}$ control theories were applied to the linearized three-dimensional channel flow. A major finding of this paper was that properly-applied controls significantly reduce the nonorthogonality leading to energy amplification mechanisms in such systems. The three-dimensional nonlinear problem was tackled by the application of optimal control theory in a finite-horizon predictive setting (Model Predictive Control) in Bewley et al. (2000), resulting in relaminarization of $R e=1700$ turbulent channel flow. Optimal controllers are generally not decentralized, but recent results on the structure of controllers for spatially-invariant systems indicate that one can obtain localized controllers arbitrarily close to optimal (Bamieh et al., 2000). This result has been confirmed for plane channel flow in Högberg and Bewley (2000), where the Fourier-space control problem formulated in Bewley and Liu (1998) was modified and successfully inverse-transformed to the physical domain, resulting in well-resolved, spatially-localized convolution kernels with exponential decay far from the origin. Such spatial localization is an important ingredient both in relaxing the nonphysical assumption of spatial periodicity in the controller formulation and in facilitating decentralized control in massive arrays of sensors and actuators (see discussion in Bewley (2001)). The mathematical details of controllability and optimal control theory applied to the Navier-Stokes equation, such as existence and uniqueness of solutions, and proofs of convergence of proposed numerical algorithms, are discussed in Abergel and Temam (1990), Barbu (1997), Barbu and Sritharan (1998), Bewley et al. (2000), Coron (1996), Coron (1998), Fattorini and Sritharan (1998), Fernández-Cara and González-Burgos (1995), Fursikov et al. (1998), Gunzburger et al. (1990), Gunzburger (1995), Hou and Yan (1997), Imanuvilov (1998), Ito and Kang (1994), Lagnese et al. (1995), Sritharan (1991), Sritharan (1998).

Global stabilization by boundary control of Burgers' equation was achieved in Krstic (1999). For the 2D case, globally stabilizing boundary control laws were presented in Balogh et al. (2001) (using wall-tangential actuation) and in Aamo et al. (2001) (using wall-normal actuation), for small Reynolds numbers. These control laws were fully decentralized, and numerical simulations showed their ability to stabilize flows at large Reynolds numbers, although the mathematical analysis was valid for small Reynolds numbers, only. As noted in Balogh et al. (2001) and Bewley (2001), fully decentralized controllers have an implementational advantage in that they can be embedded into MEMS (Micro-Electro-Mechanical-Systems) hardware, minimizing the communication requirements of centralized computations and facilitating scaling to massive arrays of sensors and actuators. Using the wall-tangential 
control law, Balogh (2001) was able to relaminarize a simulated turbulent 3D channel flow at $R e=4000$. The control law designed in Aamo et al. (2001) for wall-normal actuation can easily be generalized to both $3 \mathrm{D}$ channel flow and pipe flow.

The remainder of this tutorial treats selected works in detail, starting with a review of the governing equations of incompressible fluid flow.

\section{Governing Equations}

\subsection{Kinematics}

We will be studying the behavior of a fluid contained in the domain $\Omega$, as shown schematically in Figure 1. Associated with the fluid is its density, $\rho: \Omega \times \mathbb{R}_{+} \rightarrow \mathbb{R}$. At every time instant $t>0$, and to every point $p \in \Omega$, we assign a vector valued quantity which is the velocity, $\mathbf{W}$, of the fluid at that point in time and space. That is, we are interested in the evolution of a vector field $\mathbf{W}: \Omega \times \mathbb{R}_{+} \rightarrow \mathbb{R}^{n}$, where $n$ is the dimension of the problem. Associated with the velocity field is a pressure field, which is a scalar valued function $P: \Omega \times \mathbb{R}_{+} \rightarrow \mathbb{R}$. We will study problems in 2 and 3 spatial dimensions (2D and $3 \mathrm{D})$, using cartesian and cylindrical coordinates. In cartesian coordinates, we denote a point $p \in \Omega$ with $(x, y)$ in $2 \mathrm{D}$ and $(x, y, z)$ in 3D. In cylindrical coordinates, we denote a point $p \in \Omega$ with $(r, \theta, z)$. The two coordinate systems are shown schematically in Figure 1. The velocity field is denoted $\mathbf{W}(x, y, z, t)=(U(x, y, z, t)$, $V(x, y, z, t), W(x, y, z, t))$ in $3 \mathrm{D}$ cartesian coordinates, where $U, V$ and $W$ are the velocity components in the $x, y$ and $z$ directions, respectively $(\mathbf{W}(x, y, t)=(U(x, y, t)$, $V(x, y, t))$ in 2D). In cylindrical coordinates, we denote the velocity field $\mathbf{W}(r, \theta, z, t)=$ $\left(V_{r}(x, y, z, t), V_{\theta}(x, y, z, t), V_{z}(x, y, z, t)\right)$, where $V_{r}, V_{\theta}$, and $V_{z}$ are the velocity components in the $r, \theta$ and $z$ directions, respectively. The density, $\rho$, and the pressure, $P$, take the same arguments as the velocity, but are scalar valued. Below we will derive the conservation equations in cartesian coordinates, and state the corresponding equations in cylindrical coordinates. The derivation follows Bird et al. (1960).

\subsection{Conservation of mass}

Consider the stationary volume element in Figure 2. Writing a mass balance over the volume, we have

rate of mass accumulation $=$ rate of mass in-rate of mass out

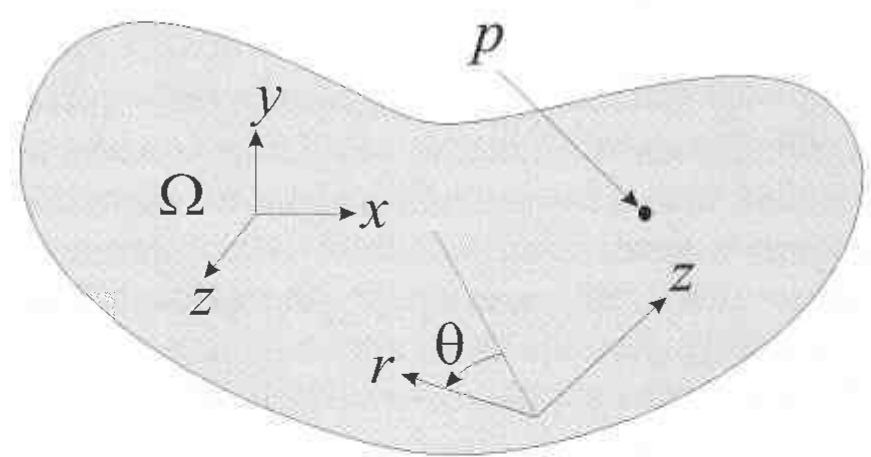

Figure 1. The domain in which the fluid is contained is denoted $\Omega$. Two coordinate systems will be used in this report: cartesian coordinates, denoted $(x, y, z)$, and cylindrical coordinates, denoted $(r, \theta, z)$. 


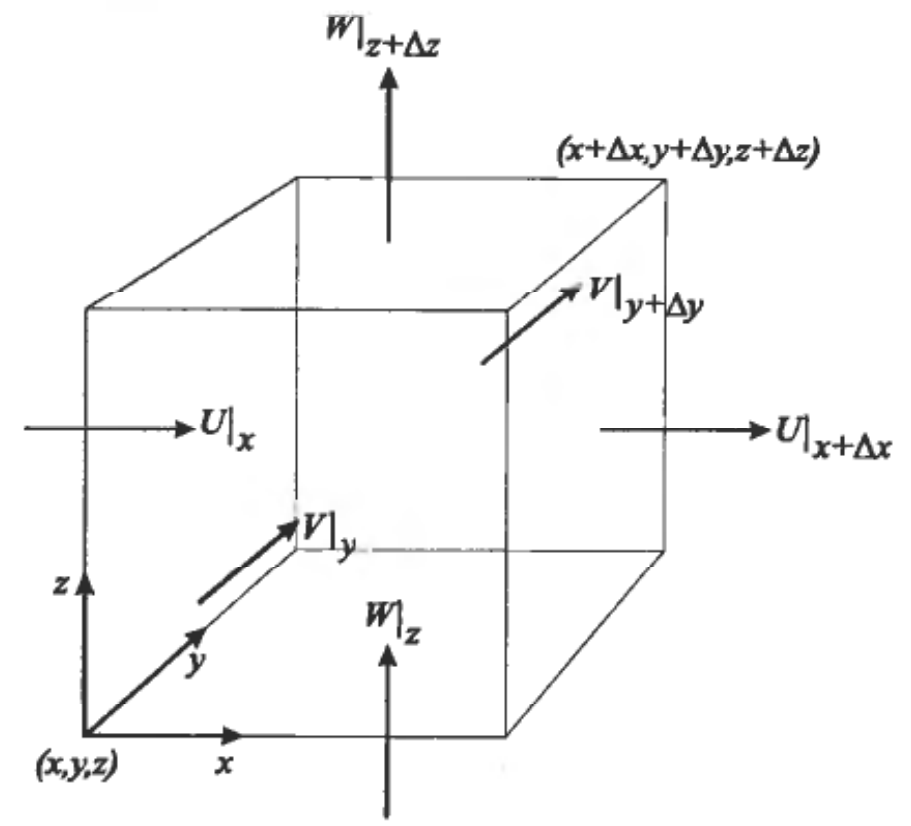

Figure 2. Control volume for derivation of the governing equations.

where

$$
\begin{aligned}
\text { rate of mass in } & =\left.(\rho U)\right|_{x} \Delta y \Delta z+\left.(\rho V)\right|_{y} \Delta x \Delta z+\left.(\rho W)\right|_{z} \Delta x \Delta y \\
\text { rate of mass out } & =\left.(\rho U)\right|_{x+\Delta x} \Delta y \Delta z+\left.(\rho V)\right|_{y+\Delta y} \Delta x \Delta z+\left.(\rho W)\right|_{z+\Delta z} \Delta y \Delta z
\end{aligned}
$$

Thus, we get

$$
\begin{aligned}
\frac{\partial \rho}{\partial t} \Delta x \Delta y \Delta z= & -\left(\left.(\rho U)\right|_{x+\Delta x}-\left.(\rho U)\right|_{x}\right) \Delta y \Delta z-\left(\left.(\rho V)\right|_{y+\Delta y}-\left.(\rho V)\right|_{y}\right) \Delta x \Delta z \\
& -\left(\left.(\rho W)\right|_{z+\Delta z}-\left.(\rho W)\right|_{z}\right) \Delta y \Delta z
\end{aligned}
$$

Dividing by the volume and letting $\Delta x, \Delta y$, and $\Delta z$ approach zero, we get

$$
\frac{\partial \rho}{\partial t}=-\frac{\partial(\rho U)}{\partial x}-\frac{\partial(\rho V)}{\partial y}-\frac{\partial(\rho W)}{\partial z} .
$$

We will be dealing exclusively with incompressible fluids, for which $\rho$ is constant. Consequently,

$$
\frac{\partial U}{\partial x}+\frac{\partial V}{\partial y}+\frac{\partial W}{\partial z}=0
$$

Equation (1) is referred to as the equation of continuity.

\subsection{Conservation of momentum}

Again consider the stationary volume element in Figure 2. Writing a momentum balance over the volume, we have 
So for the momentum in the $x$-direction, we have

$$
\begin{aligned}
\text { rate of momentum in }= & \left.\left(\rho U^{2}\right)\right|_{x} \Delta y \Delta z+\left.(\rho U V)\right|_{y} \Delta x \Delta z+\left.(\rho U W)\right|_{z} \Delta x \Delta y \\
\text { rate of momentum out }= & \left.\left(\rho U^{2}\right)\right|_{x+\Delta x} \Delta y \Delta z+\left.(\rho U V)\right|_{y+\Delta y} \Delta x \Delta z \\
& +\left.(\rho U W)\right|_{z+\Delta z} \Delta x \Delta y
\end{aligned}
$$

sum of forces acting on system $=\left(\left.\tau_{x x}\right|_{x}-\left.\tau_{x x}\right|_{x+\Delta x}\right) \Delta y \Delta z+\left(\left.\tau_{y x}\right|_{y}-\left.\tau_{y x}\right|_{y+\Delta y}\right) \Delta x \Delta z$

$$
\begin{aligned}
& +\left(\left.\tau_{z x}\right|_{z} \Delta x \Delta y-\left.\tau_{z x}\right|_{z+\Delta z}\right) \Delta x \Delta y \\
& +\left(\left.P\right|_{x}-\left.P\right|_{x+\Delta x}\right) \Delta y \Delta z
\end{aligned}
$$

where $\tau_{i j}$ denotes the viscous force (per unit area) acting in the direction of $j$ on a face normal to the $i$-direction, and $P$ is the pressure (i.e. the pressure force per unit area). We get

$$
\begin{aligned}
\frac{\partial(\rho U)}{\partial t} \Delta x \Delta y \Delta z= & -\left(\left.\left(\rho U^{2}\right)\right|_{x+\Delta x}-\left.\left(\rho U^{2}\right)\right|_{x}\right) \Delta y \Delta z-\left(\left.(\rho U V)\right|_{y+\Delta y}-\left.(\rho U V)\right|_{y}\right) \Delta x \Delta z \\
& -\left(\left.(\rho U W)\right|_{z+\Delta z}-\left.(\rho U W)\right|_{z}\right) \Delta x \Delta y-\left(\left.\tau_{x x}\right|_{x+\Delta x}-\left.\tau_{x x}\right|_{x}\right) \Delta y \Delta z \\
& -\left(\left.\tau_{y x}\right|_{y+\Delta y}-\left.\tau_{y x}\right|_{y}\right) \Delta x \Delta z-\left(\left.\tau_{z x}\right|_{z+\Delta z}-\left.\tau_{z x}\right|_{z}\right) \Delta x \Delta y \\
& -\left(\left.P\right|_{x+\Delta x}-\left.P\right|_{x}\right) \Delta y \Delta z
\end{aligned}
$$

Dividing by the volume and letting $\Delta x, \Delta y$, and $\Delta z$ approach zero, we get

$$
\frac{\partial(\rho U)}{\partial t}+\frac{\partial\left(\rho U^{2}\right)}{\partial x}+\frac{\partial(\rho U V)}{\partial y}+\frac{\partial(\rho U W)}{\partial z}=-\frac{\partial P}{\partial x}-\frac{\partial \tau_{x x}}{\partial x}-\frac{\partial \tau_{y x}}{\partial y}-\frac{\partial \tau_{z x}}{\partial z}
$$

For constant density, $\rho$, we get

$$
\begin{aligned}
& \rho \frac{\partial U}{\partial t}+\rho\left(U \frac{\partial U}{\partial x}+V \frac{\partial U}{\partial y}+W \frac{\partial U}{\partial z}\right)+\rho U\left(\frac{\partial U}{\partial x}+\frac{\partial V}{\partial y}+\frac{\partial W}{\partial z}\right) \\
& =-\frac{\partial P}{\partial x}-\frac{\partial \tau_{x x}}{\partial x}-\frac{\partial \tau_{y x}}{\partial y}-\frac{\partial \tau_{z x}}{\partial z}
\end{aligned}
$$

and using equation (1) yields

$$
\rho \frac{\partial U}{\partial t}+\rho\left(U \frac{\partial U}{\partial x}+V \frac{\partial U}{\partial y}+W \frac{\partial U}{\partial z}\right)=-\frac{\partial P}{\partial x}-\frac{\partial \tau_{x x}}{\partial x}-\frac{\partial \tau_{y x}}{\partial y}-\frac{\partial \tau_{z x}}{\partial z}
$$

Similar derivations for the momentum in the $y$ and $z$ directions yield the complete set of equations

$$
\begin{aligned}
& \rho \frac{\partial U}{\partial t}+\rho\left(U \frac{\partial U}{\partial x}+V \frac{\partial U}{\partial y}+W \frac{\partial U}{\partial z}\right)=-\frac{\partial P}{\partial x}-\frac{\partial \tau_{x x}}{\partial x}-\frac{\partial \tau_{y x}}{\partial y}-\frac{\partial \tau_{z x}}{\partial z} \\
& \rho \frac{\partial V}{\partial t}+\rho\left(U \frac{\partial V}{\partial x}+V \frac{\partial V}{\partial y}+W \frac{\partial V}{\partial z}\right)=-\frac{\partial P}{\partial y}-\frac{\partial \tau_{x y}}{\partial x}-\frac{\partial \tau_{y y}}{\partial y}-\frac{\partial \tau_{z y}}{\partial z}
\end{aligned}
$$




$$
\rho \frac{\partial W}{\partial t}+\rho\left(U \frac{\partial W}{\partial x}+V \frac{\partial W}{\partial y}+W \frac{\partial W}{\partial z}\right)=-\frac{\partial P}{\partial z}-\frac{\partial \tau_{x z}}{\partial x}-\frac{\partial \tau_{y z}}{\partial y}-\frac{\partial \tau_{z z}}{\partial z}
$$

It remains to insert a constitutive equation relating the viscous forces $\tau_{i j}$ to the fluid motion $\mathbf{W}=(U, V, W)$. We will exclusively consider Newtonian fluids, which in conjunction with incompressibility yield the following relations (Bird et al., 1960)

$$
\begin{aligned}
& \tau_{x x}=-2 \mu \frac{\partial U}{\partial x}, \tau_{y y}=-2 \mu \frac{\partial V}{\partial y}, \tau_{z z}=-2 \mu \frac{\partial W}{\partial z} \\
& \tau_{x y}=\tau_{y x}=-\mu\left(\frac{\partial U}{\partial y}+\frac{\partial V}{\partial x}\right) \\
& \tau_{y z}=\tau_{z y}=-\mu\left(\frac{\partial V}{\partial z}+\frac{\partial W}{\partial y}\right) \\
& \tau_{z x}=\tau_{x z}=-\mu\left(\frac{\partial W}{\partial x}+\frac{\partial U}{\partial z}\right)
\end{aligned}
$$

where the coefficient $\mu$ is called the viscosity of the fluid. Inserting equations (5)-(8) into (2)-(4) yields

$$
\begin{aligned}
\rho \frac{\partial U}{\partial t}+\rho\left(U \frac{\partial U}{\partial x}+V \frac{\partial U}{\partial y}+W \frac{\partial U}{\partial z}\right)= & -\frac{\partial P}{\partial x}+\mu\left(\frac{\partial^{2} U}{\partial x^{2}}+\frac{\partial^{2} U}{\partial y^{2}}+\frac{\partial^{2} U}{\partial z^{2}}\right) \\
& +\mu \frac{\partial}{\partial x}\left(\frac{\partial U}{\partial x}+\frac{\partial V}{\partial y}+\frac{\partial W}{\partial z}\right) \\
\rho \frac{\partial V}{\partial t}+\rho\left(U \frac{\partial V}{\partial x}+V \frac{\partial V}{\partial y}+W \frac{\partial V}{\partial z}\right)= & -\frac{\partial P}{\partial y}+\mu\left(\frac{\partial^{2} V}{\partial x^{2}}+\frac{\partial^{2} V}{\partial y^{2}}+\frac{\partial^{2} V}{\partial z^{2}}\right) \\
& +\mu \frac{\partial}{\partial y}\left(\frac{\partial U}{\partial x}+\frac{\partial V}{\partial y}+\frac{\partial W}{\partial z}\right) \\
\rho \frac{\partial W}{\partial t}+\rho\left(U \frac{\partial W}{\partial x}+V \frac{\partial W}{\partial y}+W \frac{\partial W}{\partial z}\right)= & -\frac{\partial P}{\partial z}+\mu\left(\frac{\partial^{2} W}{\partial x^{2}}+\frac{\partial^{2} W}{\partial y^{2}}+\frac{\partial^{2} W}{\partial z^{2}}\right) \\
& +\mu \frac{\partial}{\partial z}\left(\frac{\partial U}{\partial x}+\frac{\partial V}{\partial y}+\frac{\partial W}{\partial z}\right)
\end{aligned}
$$

and using equation (1) we finally get

$$
\rho\left(\frac{\partial U}{\partial t}+U \frac{\partial U}{\partial x}+V \frac{\partial U}{\partial y}+W \frac{\partial U}{\partial z}\right)=-\frac{\partial P}{\partial x}+\mu\left(\frac{\partial^{2} U}{\partial x^{2}}+\frac{\partial^{2} U}{\partial y^{2}}+\frac{\partial^{2} U}{\partial z^{2}}\right)
$$




$$
\begin{gathered}
\rho\left(\frac{\partial V}{\partial t}+U \frac{\partial V}{\partial x}+V \frac{\partial V}{\partial y}+W \frac{\partial V}{\partial z}\right)=-\frac{\partial p}{\partial y}+\mu\left(\frac{\partial^{2} V}{\partial x^{2}}+\frac{\partial^{2} V}{\partial y^{2}}+\frac{\partial^{2} V}{\partial z^{2}}\right) \\
\rho\left(\frac{\partial W}{\partial t}+U \frac{\partial W}{\partial x}+V \frac{\partial W}{\partial y}+W \frac{\partial W}{\partial z}\right)=-\frac{\partial P}{\partial z}+\mu\left(\frac{\partial^{2} W}{\partial x^{2}}+\frac{\partial^{2} W}{\partial y^{2}}+\frac{\partial^{2} W}{\partial z^{2}}\right)
\end{gathered}
$$

Equations (9)-(11) is the celebrated Navier-Stokes equation. In vector form, equations (1) and (9)-(11) can be written compactly as

$$
\begin{array}{r}
\operatorname{div}(\mathbf{W})=0 \\
\frac{\partial \mathbf{W}}{\partial t}+(\mathbf{W} \cdot \nabla) \mathbf{W}=-\frac{1}{\rho} \nabla P+\frac{\mu}{\rho} \Delta \mathbf{W}
\end{array}
$$

where $\nabla$ denotes the gradient operator, $\Delta$ denotes the Laplace operator, and div is short for divergence.

\subsection{The non-dimensionalized Navier-Stokes equation}

2.4.1. Cartesian coordinates For many flow geometries, the Navier-Stokes equations can be written in non-dimensionalized form by introduction of a characteristic length and a characteristic velocity. In the resulting form of the equations, all physical parameters (fluid properties and geometrical constants) are lumped into one parameter, which characterizes the behaviour of the flow. This simplifies exploration of the parameter space considerably. Denoting the characteristic length $\breve{D}$, and the characteristic velocity $\breve{V}$, we can do a change of variables in such a way that the new variables are dimensionless

$$
\begin{gathered}
\left(U^{*}, V^{*}, W^{*}\right)=\left(\frac{U}{\breve{V}}, \frac{V}{\breve{V}}, \frac{W}{\breve{V}}\right), P^{*}=\frac{P}{\rho \breve{V}^{2}}, t^{*}=\frac{\breve{V}}{\breve{D}} t \\
x^{*}=\frac{x}{\check{D}}, y^{*}=\frac{y}{\breve{D}}, z^{*}=\frac{z}{\check{D}}
\end{gathered}
$$

So we get

$$
\begin{aligned}
\frac{\partial U}{\partial t} & =\breve{V} \frac{\partial U^{*}}{\partial t^{*}} \frac{\partial t^{*}}{\partial t}=\frac{\breve{V}^{2}}{\breve{D}} \frac{\partial U^{*}}{\partial t^{*}} \\
\frac{\partial U}{\partial x} & =\breve{V} \frac{\partial U^{*}}{\partial x^{*}} \frac{\partial x^{*}}{\partial x}=\frac{\breve{V}}{\breve{D}} \frac{\partial U^{*}}{\partial x^{*}} \\
\frac{\partial P}{\partial x} & =\rho \breve{V}^{2} \frac{\partial P^{*}}{\partial x^{*}} \frac{\partial x^{*}}{\partial x}=\frac{\rho \breve{V}^{2}}{\breve{D}} \frac{\partial P^{*}}{\partial x^{*}} \\
\frac{\partial^{2} U}{\partial x^{2}} & =\frac{\partial}{\partial x^{*}}\left(\frac{\breve{V}}{\breve{D}} \frac{\partial U^{*}}{\partial x^{*}}\right) \frac{\partial x^{*}}{\partial x}=\frac{\breve{V}}{\breve{D}^{2}} \frac{\partial^{2} U^{*}}{\partial x^{* 2}}
\end{aligned}
$$


and similarly for the other derivatives occuring in equations (1) and (9)-(11). Inserting these relations into equations (1) and (9)-(11) yields

$$
\begin{aligned}
& \frac{\partial U}{\partial x}+\frac{\partial V}{\partial y}+\frac{\partial W}{\partial z}=0 \\
& \frac{\partial U}{\partial t}+U \frac{\partial U}{\partial x}+V \frac{\partial U}{\partial y}+W \frac{\partial U}{\partial z}=-\frac{\partial P}{\partial x}+\frac{1}{R e}\left(\frac{\partial^{2} U}{\partial x^{2}}+\frac{\partial^{2} U}{\partial y^{2}}+\frac{\partial^{2} U}{\partial z^{2}}\right) \\
& \frac{\partial V}{\partial t}+U \frac{\partial V}{\partial x}+V \frac{\partial V}{\partial y}+W \frac{\partial V}{\partial z}=-\frac{\partial P}{\partial y}+\frac{1}{R e}\left(\frac{\partial^{2} V}{\partial x^{2}}+\frac{\partial^{2} V}{\partial y^{2}}+\frac{\partial^{2} V}{\partial z^{2}}\right) \\
& \frac{\partial W}{\partial t}+U \frac{\partial W}{\partial x}+V \frac{\partial W}{\partial y}+W \frac{\partial W}{\partial z}=-\frac{\partial P}{\partial z}+\frac{1}{R e}\left(\frac{\partial^{2} W}{\partial x^{2}}+\frac{\partial^{2} W}{\partial y^{2}}+\frac{\partial^{2} W}{\partial z^{2}}\right)
\end{aligned}
$$

where we have defined $R e=\rho \check{D} \check{V} / \mu$, and skipped the superscript * for notational convenience. $R e$ is called the Reynolds number, and is the only parameter in equations (13)-(15). In vector notation, equations (12) and (13)-(15) become

$$
\begin{gathered}
\operatorname{div}(\mathbf{W})=0 \\
\frac{\partial \mathbf{W}}{\partial t}+(\mathbf{W} \cdot \nabla) \mathbf{W}=-\nabla P+\frac{1}{R e} \Delta \mathbf{W}
\end{gathered}
$$

2.4.2. Cylindrical coordinates A good choice of coordinate system may simplify mathematical analysis substantially. It is usually convenient to select coordinates such that domain boundaries coincide with surfaces on which one coordinate is constant. Thus, the obvious choice of coordinates for pipe flow is cylindrical cooridnates. In cylindrical coordinates, equations (12) (15) become (see, for instance, Bird et al. (1960)),

$$
\begin{aligned}
& \frac{1}{r} \frac{\partial}{\partial r}\left(r V_{r}\right)+\frac{1}{r} \frac{\partial V_{\theta}}{\partial \theta}+\frac{\partial V_{z}}{\partial z}=0 \\
& \frac{\partial V_{r}}{\partial t}+V_{r} \frac{\partial V_{r}}{\partial r}+\frac{V_{\theta}}{r} \frac{\partial V_{r}}{\partial \theta}-\frac{V_{\theta}^{2}}{r}+V_{z} \frac{\partial V_{r}}{\partial z}=-\frac{\partial P}{\partial r}+\frac{1}{R e}\left(\frac{\partial}{\partial r}\left(\frac{1}{r} \frac{\partial}{\partial r}\left(r V_{r}\right)\right)\right. \\
& \left.+\frac{1}{r^{2}} \frac{\partial^{2} V_{r}}{\partial \theta^{2}}-\frac{2}{r^{2}} \frac{\partial V_{\theta}}{\partial \theta}+\frac{\partial^{2} V_{r}}{\partial z^{2}}\right) \\
& \frac{\partial V_{\theta}}{\partial t}+V_{r} \frac{\partial V_{\theta}}{\partial r}+\frac{V_{\theta}}{r} \frac{\partial V_{\theta}}{\partial \theta}+\frac{V_{r} V_{\theta}}{r}+V_{z} \frac{\partial V_{\theta}}{\partial z}=-\frac{1}{r} \frac{\partial P}{\partial \theta}+\frac{1}{R e}\left(\frac{\partial}{\partial r}\left(\frac{1}{r} \frac{\partial}{\partial r}\left(r V_{\theta}\right)\right)\right. \\
& \left.+\frac{1}{r^{2}} \frac{\partial^{2} V_{\theta}}{\partial \theta^{2}}+\frac{2}{r^{2}} \frac{\partial V_{r}}{\partial \theta}+\frac{\partial^{2} V_{\theta}}{\partial z^{2}}\right)
\end{aligned}
$$




$$
\frac{\partial V_{z}}{\partial t}+V_{r} \frac{\partial V_{z}}{\partial r}+\frac{V_{\theta}}{r} \frac{\partial V_{z}}{\partial \theta}+V_{z} \frac{\partial V_{z}}{\partial z}=-\frac{\partial P}{\partial z}+\frac{1}{R e}\left(\frac{1}{r} \frac{\partial}{\partial r}\left(r \frac{\partial V_{z}}{\partial r}\right)+\frac{1}{r^{2}} \frac{\partial^{2} V_{z}}{\partial \theta^{2}}+\frac{\partial^{2} V_{z}}{\partial z^{2}}\right)
$$

\subsection{Perturbations and the linearized Navier-Stokes equations}

2.5.1. Cartesian coordinates Suppose $(\tilde{U}, \tilde{V}, \tilde{W}, \tilde{P})$ is a steady state solution of equations (12) and (13)-(15), that is

$$
\left(\frac{\partial \tilde{U}}{\partial t}, \frac{\partial \tilde{V}}{\partial t}, \frac{\partial \tilde{W}}{\partial t}, \frac{\partial \tilde{P}}{\partial t}\right)=(0,0,0,0)
$$

and

$$
\begin{aligned}
& \frac{\partial \tilde{U}}{\partial x}+\frac{\partial \tilde{V}}{\partial y}+\frac{\partial \tilde{W}}{\partial z}=0 \\
& \tilde{U} \frac{\partial \tilde{U}}{\partial x}+\tilde{V} \frac{\partial \tilde{U}}{\partial y}+\tilde{W} \frac{\partial \tilde{U}}{\partial z}=-\frac{\partial \tilde{P}}{\partial x}+\frac{1}{R e}\left(\frac{\partial^{2} \tilde{U}}{\partial x^{2}}+\frac{\partial^{2} \tilde{U}}{\partial y^{2}}+\frac{\partial^{2} \tilde{U}}{\partial z^{2}}\right) \\
& \tilde{U} \frac{\partial \tilde{V}}{\partial x}+\tilde{V} \frac{\partial \tilde{V}}{\partial y}+\tilde{W} \frac{\partial \tilde{V}}{\partial z}=-\frac{\partial \tilde{P}}{\partial y}+\frac{1}{R e}\left(\frac{\partial^{2} \tilde{V}}{\partial x^{2}}+\frac{\partial^{2} \tilde{V}}{\partial y^{2}}+\frac{\partial^{2} \tilde{V}}{\partial z^{2}}\right) \\
& \tilde{U} \frac{\partial \tilde{W}}{\partial x}+\tilde{V} \frac{\partial \tilde{W}}{\partial y}+\tilde{W} \frac{\partial \tilde{W}}{\partial z}=-\frac{\partial \tilde{P}}{\partial z}+\frac{1}{R e}\left(\frac{\partial^{2} \tilde{W}}{\partial x^{2}}+\frac{\partial^{2} \tilde{W}}{\partial y^{2}}+\frac{\partial^{2} \tilde{W}}{\partial z^{2}}\right)
\end{aligned}
$$

Defining the perturbations $\mathbf{w}=(u, v, w)$ and $p$ as

$$
\begin{aligned}
& u \triangleq U-\tilde{U} \\
& v \triangleq V-\tilde{V} \\
& w \triangleq W-\tilde{W} \\
& p \triangleq P-\tilde{P}
\end{aligned}
$$

so that

$$
\begin{gathered}
U=u+\tilde{U} \\
V=v+\tilde{V} \\
W=w+\tilde{W} \\
P=p+\tilde{P}
\end{gathered}
$$

and inserting into equations (12) and (13)-(15), yield

$$
\frac{\partial u}{\partial x}+\frac{\partial v}{\partial y}+\frac{\partial w}{\partial z}+\frac{\partial \tilde{U}}{\partial x}+\frac{\partial \tilde{V}}{\partial y}+\frac{\partial \tilde{W}}{\partial z}=0
$$




$$
\begin{gathered}
\frac{\partial u}{\partial t}+\frac{\partial \tilde{U}}{\partial t}+u \frac{\partial u}{\partial x}+u \frac{\partial \tilde{U}}{\partial x}+\tilde{U} \frac{\partial u}{\partial x}+v \frac{\partial u}{\partial y}+v \frac{\partial \tilde{U}}{\partial y} \\
+\tilde{V} \frac{\partial u}{\partial y}+w \frac{\partial u}{\partial z}+w \frac{\partial \tilde{U}}{\partial z}+\tilde{W} \frac{\partial u}{\partial z}+\tilde{U} \frac{\partial \tilde{U}}{\partial x}+\tilde{V} \frac{\partial \tilde{U}}{\partial y}+\tilde{W} \frac{\partial \tilde{U}}{\partial z} \\
=-\frac{\partial p}{\partial x}-\frac{\partial \tilde{P}}{\partial x}+\frac{1}{R e}\left(\frac{\partial^{2} u}{\partial x^{2}}+\frac{\partial^{2} u}{\partial y^{2}}+\frac{\partial^{2} u}{\partial z^{2}}+\frac{\partial^{2} \tilde{U}}{\partial x^{2}}+\frac{\partial^{2} \tilde{U}}{\partial y^{2}}+\frac{\partial^{2} \tilde{U}}{\partial z^{2}}\right) \\
\frac{\partial v}{\partial t}+\frac{\partial \tilde{V}}{\partial t}+u \frac{\partial v}{\partial x}+\tilde{U} \frac{\partial v}{\partial x}+\frac{\partial \tilde{V}}{\partial x} u+v \frac{\partial v}{\partial y}+v \frac{\partial \tilde{V}}{\partial y} \\
+\tilde{V} \frac{\partial v}{\partial y}+w \frac{\partial v}{\partial z}+w \frac{\partial \tilde{V}}{\partial z}+\tilde{W} \frac{\partial v}{\partial z}+\tilde{U} \frac{\partial \tilde{V}}{\partial x}+\tilde{V} \frac{\partial \tilde{V}}{\partial y}+\tilde{W} \frac{\partial \tilde{V}}{\partial z} \\
=-\frac{\partial p}{\partial y}-\frac{\partial \tilde{P}}{\partial y}+\frac{1}{R e}\left(\frac{\partial^{2} v}{\partial x^{2}}+\frac{\partial^{2} v}{\partial y^{2}}+\frac{\partial^{2} v}{\partial z^{2}}+\frac{\partial^{2} \tilde{V}}{\partial x^{2}}+\frac{\partial^{2} \tilde{V}}{\partial y^{2}}+\frac{\partial^{2} \tilde{V}}{\partial z^{2}}\right)
\end{gathered}
$$

$$
\begin{aligned}
\frac{\partial w}{\partial t}+ & \frac{\partial \tilde{W}}{\partial t}+u \frac{\partial w}{\partial x}+u \frac{\partial \tilde{W}}{\partial x}+\tilde{U} \frac{\partial w}{\partial x}+v \frac{\partial w}{\partial y}+v \frac{\partial \tilde{W}}{\partial y} \\
& +\tilde{V} \frac{\partial w}{\partial y}+w \frac{\partial w}{\partial z}+w \frac{\partial \tilde{W}}{\partial z}+\tilde{W} \frac{\partial w}{\partial z}+\tilde{U} \frac{\partial \tilde{W}}{\partial x}+\tilde{V} \frac{\partial \tilde{W}}{\partial y}+\tilde{W} \frac{\partial \tilde{W}}{\partial z} \\
= & -\frac{\partial p}{\partial z}-\frac{\partial \tilde{P}}{\partial z}+\frac{1}{R e}\left(\frac{\partial^{2} w}{\partial x^{2}}+\frac{\partial^{2} w}{\partial y^{2}}+\frac{\partial^{2} w}{\partial z^{2}}+\frac{\partial^{2} \tilde{W}}{\partial x^{2}}+\frac{\partial^{2} \tilde{W}}{\partial y^{2}}+\frac{\partial^{2} \tilde{W}}{\partial z^{2}}\right)
\end{aligned}
$$

In view of equations (20)-(24), we get the perturbation equations

$$
\begin{aligned}
& \frac{\partial u}{\partial x}+\frac{\partial v}{\partial y}+\frac{\partial w}{\partial z}=0 \\
& \frac{\partial u}{\partial t}+u \frac{\partial u}{\partial x}+u \frac{\partial \tilde{U}}{\partial x}+\tilde{U} \frac{\partial u}{\partial x}+v \frac{\partial u}{\partial y}+v \frac{\partial \tilde{U}}{\partial y}+\tilde{V} \frac{\partial u}{\partial y}+w \frac{\partial u}{\partial z}+w \frac{\partial \tilde{U}}{\partial z}+\tilde{W} \frac{\partial u}{\partial z} \\
& =-\frac{\partial p}{\partial x}+\frac{1}{R e}\left(\frac{\partial^{2} u}{\partial x^{2}}+\frac{\partial^{2} u}{\partial y^{2}}+\frac{\partial^{2} u}{\partial z^{2}}\right) \\
& \frac{\partial v}{\partial t}+u \frac{\partial v}{\partial x}+\tilde{U} \frac{\partial v}{\partial x}+\frac{\partial \tilde{V}}{\partial x} u+v \frac{\partial v}{\partial y}+v \frac{\partial \tilde{V}}{\partial y}+\tilde{\boldsymbol{V}} \frac{\partial v}{\partial y}+w \frac{\partial v}{\partial z}+w \frac{\partial \tilde{\boldsymbol{V}}}{\partial z}+\tilde{W} \frac{\partial v}{\partial z} \\
& =-\frac{\partial p}{\partial y}+\frac{1}{R e}\left(\frac{\partial^{2} v}{\partial x^{2}}+\frac{\partial^{2} v}{\partial y^{2}}+\frac{\partial^{2} v}{\partial z^{2}}\right) \\
& \frac{\partial w}{\partial t}+u \frac{\partial w}{\partial x}+u \frac{\partial \tilde{W}}{\partial x}+\tilde{U} \frac{\partial w}{\partial x}+v \frac{\partial w}{\partial y}+v \frac{\partial \tilde{W}}{\partial y}+\tilde{V} \frac{\partial w}{\partial y}+w \frac{\partial w}{\partial z}+w \frac{\partial \tilde{W}}{\partial z}+\tilde{W} \frac{\partial w}{\partial z} \\
& =-\frac{\partial p}{\partial z}+\frac{1}{R e}\left(\frac{\partial^{2} w}{\partial x^{2}}+\frac{\partial^{2} w}{\partial y^{2}}+\frac{\partial^{2} w}{\partial z^{2}}\right)
\end{aligned}
$$


The linearized equations around the steady state solution $(\tilde{U}, \tilde{V}, \tilde{W}, \tilde{P})$ are now obtained by omitting terms that are second order in the perturbations. Thus, we get

$$
\begin{gathered}
\frac{\partial u}{\partial x}+\frac{\partial v}{\partial y}+\frac{\partial w}{\partial z}=0 \\
\frac{\partial u}{\partial t}+u \frac{\partial \tilde{U}}{\partial x}+\tilde{U} \frac{\partial u}{\partial x}+v \frac{\partial \tilde{U}}{\partial y}+\tilde{V} \frac{\partial u}{\partial y}+w \frac{\partial \tilde{U}}{\partial z}+\tilde{W} \frac{\partial u}{\partial z} \\
=-\frac{\partial p}{\partial x}+\frac{1}{R e}\left(\frac{\partial^{2} u}{\partial x^{2}}+\frac{\partial^{2} u}{\partial y^{2}}+\frac{\partial^{2} u}{\partial z^{2}}\right) \\
\frac{\partial v}{\partial t}+\tilde{U} \frac{\partial v}{\partial x}+\frac{\partial \tilde{V}}{\partial x} u+v \frac{\partial \tilde{V}}{\partial y}+\tilde{V} \frac{\partial v}{\partial y}+w \frac{\partial \tilde{V}}{\partial z}+\tilde{W} \frac{\partial v}{\partial z} \\
=-\frac{\partial p}{\partial y}+\frac{1}{R e}\left(\frac{\partial^{2} v}{\partial x^{2}}+\frac{\partial^{2} v}{\partial y^{2}}+\frac{\partial^{2} v}{\partial z^{2}}\right) \\
\frac{\partial w}{\partial t}+u \frac{\partial \tilde{W}}{\partial x}+\tilde{U} \frac{\partial w}{\partial x}+v \frac{\partial \tilde{W}}{\partial y}+\tilde{V} \frac{\partial w}{\partial y}+w \frac{\partial \tilde{W}}{\partial z}+\tilde{W} \frac{\partial w}{\partial z} \\
=-\frac{\partial p}{\partial z}+\frac{1}{R e}\left(\frac{\partial^{2} w}{\partial x^{2}}+\frac{\partial^{2} w}{\partial y^{2}}+\frac{\partial^{2} w}{\partial z^{2}}\right)
\end{gathered}
$$

2.5.2. Cylindrical coordinates Suppose $\left(\tilde{V}_{r}, \tilde{V}_{\theta}, \tilde{V}_{z}, \tilde{P}\right)$ is a steady state solution of equations (16) and (17)-(19). Defining the perturbation

$$
\begin{aligned}
& v_{r} \triangleq V_{r}-\tilde{V}_{r} \\
& v_{\theta} \triangleq V_{\theta}-\tilde{V}_{\theta} \\
& v_{z} \triangleq V_{z}-\tilde{V}_{z} \\
& p \triangleq P-\tilde{P}
\end{aligned}
$$

we get the perturbation equations

$$
\begin{gathered}
\frac{1}{r} \frac{\partial}{\partial r}\left(r v_{r}\right)+\frac{1}{r} \frac{\partial v_{\theta}}{\partial \theta}+\frac{\partial v_{z}}{\partial z}=0 \\
\frac{\partial v_{r}}{\partial t}+v_{r} \frac{\partial v_{r}}{\partial r}+v_{r} \frac{\partial \tilde{V}_{r}}{\partial r}+\tilde{V}_{r} \frac{\partial v_{r}}{\partial r}+\frac{v_{\theta}}{r} \frac{\partial v_{r}}{\partial \theta}+\frac{v_{\theta}}{r} \frac{\partial \tilde{V}_{r}}{\partial \theta} \\
+\frac{\tilde{V}_{\theta}}{r} \frac{\partial v_{r}}{\partial \theta}-\frac{v_{\theta}^{2}}{r}-2 \frac{v_{\theta}}{r} \tilde{V}_{\theta}+v_{z} \frac{\partial v_{r}}{\partial z}+v_{z} \frac{\partial \tilde{V}_{r}}{\partial z}+\tilde{V}_{z} \frac{\partial v_{r}}{\partial z} \\
=-\frac{\partial p}{\partial r}+\frac{1}{\operatorname{Re}}\left(\frac{\partial}{\partial r}\left(\frac{1}{r} \frac{\partial}{\partial r}\left(r v_{r}\right)\right)+\frac{1}{r^{2}} \frac{\partial^{2} v_{r}}{\partial \theta^{2}}-\frac{2}{r^{2}} \frac{\partial v_{\theta}}{\partial \theta}+\frac{\partial^{2} v_{r}}{\partial z^{2}}\right)
\end{gathered}
$$




$$
\begin{aligned}
& \frac{\partial v_{\theta}}{\partial t}+v_{r} \frac{\partial v_{\theta}}{\partial r}+v_{r} \frac{\partial \tilde{V}_{\theta}}{\partial r}+\tilde{V}_{r} \frac{\partial v_{\theta}}{\partial r}+\frac{v_{\theta}}{r} \frac{\partial v_{\theta}}{\partial \theta}+\frac{v_{\theta}}{r} \frac{\partial \tilde{V}_{\theta}}{\partial \theta}+\frac{\tilde{V}_{\theta}}{r} \frac{\partial v_{\theta}}{\partial \theta} \\
& +\frac{v_{r} v_{\theta}}{r}+\frac{v_{r} V_{\theta}}{r}+\frac{V_{r} v_{\theta}}{r}+v_{z} \frac{\partial v_{\theta}}{\partial z}+v_{z} \frac{\partial \tilde{V}_{\theta}}{\partial z}+\tilde{V}_{z} \frac{\partial v_{\theta}}{\partial z} \\
& =-\frac{1}{r} \frac{\partial p}{\partial \theta}+\frac{1}{R e}\left(\frac{\partial}{\partial r}\left(\frac{1}{r} \frac{\partial}{\partial r}\left(r v_{\theta}\right)\right)+\frac{1}{r^{2}} \frac{\partial^{2} v_{\theta}}{\partial \theta^{2}}+\frac{2}{r^{2}} \frac{\partial v_{r}}{\partial \theta}+\frac{\partial^{2} v_{\theta}}{\partial z^{2}}\right) \\
& \frac{\partial v_{z}}{\partial t}+v_{r} \frac{\partial v_{z}}{\partial r}+v_{r} \frac{\partial \tilde{V}_{z}}{\partial r}+\tilde{V}_{r} \frac{\partial v_{z}}{\partial r}+\frac{v_{\theta}}{r} \frac{\partial v_{z}}{\partial \theta}+\frac{v_{\theta}}{r} \frac{\partial \tilde{V}_{z}}{\partial \theta}+\frac{\tilde{V}_{\theta}}{r} \frac{\partial v_{z}}{\partial \theta}+v_{z} \frac{\partial v_{z}}{\partial z}+v_{z} \frac{\partial \tilde{V}_{z}}{\partial z}+\tilde{V}_{z} \frac{\partial v_{z}}{\partial z} \\
& =-\frac{\partial p}{\partial z}+\frac{1}{\operatorname{Re}}\left(\frac{1}{r} \frac{\partial}{\partial r}\left(r \frac{\partial v_{z}}{\partial r}\right)+\frac{1}{r^{2}} \frac{\partial^{2} v_{z}}{\partial \theta^{2}}+\frac{\partial^{2} v_{z}}{\partial z^{2}}\right)
\end{aligned}
$$

and the linearized equations around the steady state solution $\left(\tilde{V}_{r}, \tilde{V}_{\theta}, \tilde{V}_{z}, \tilde{P}\right)$

$$
\begin{aligned}
& \frac{1}{r} \frac{\partial}{\partial r}\left(r v_{r}\right)+\frac{1}{r} \frac{\partial v_{\theta}}{\partial \theta}+\frac{\partial v_{z}}{\partial z}=0 \\
& \frac{\partial v_{r}}{\partial t}+v_{r} \frac{\partial \tilde{V}_{r}}{\partial r}+\tilde{V}_{r} \frac{\partial v_{r}}{\partial r}+\frac{v_{\theta}}{r} \frac{\partial \tilde{V}_{r}}{\partial \theta}+\frac{\tilde{V}_{\theta}}{r} \frac{\partial v_{r}}{\partial \theta}-2 \frac{v_{\theta}}{r} \tilde{V}_{\theta}+v_{z} \frac{\partial \tilde{V}_{r}}{\partial z}+\tilde{V}_{z} \frac{\partial v_{r}}{\partial z} \\
& =-\frac{\partial p}{\partial r}+\frac{1}{R e}\left(\frac{\partial}{\partial r}\left(\frac{1}{r} \frac{\partial}{\partial r}\left(r v_{r}\right)\right)+\frac{1}{r^{2}} \frac{\partial^{2} v_{r}}{\partial \theta^{2}}-\frac{2}{r^{2}} \frac{\partial v_{\theta}}{\partial \theta}+\frac{\partial^{2} v_{r}}{\partial z^{2}}\right) \\
& \frac{\partial v_{\theta}}{\partial t}+v_{r} \frac{\partial \tilde{V}_{\theta}}{\partial r}+\tilde{V}_{r} \frac{\partial v_{\theta}}{\partial r}+\frac{v_{\theta}}{r} \frac{\partial \tilde{V}_{\theta}}{\partial \theta}+\frac{\tilde{V}_{\theta}}{r} \frac{\partial v_{\theta}}{\partial \theta}+\frac{v_{r} V_{\theta}}{r}+\frac{V_{r} v_{\theta}}{r}+v_{s} \frac{\partial \tilde{V}_{\theta}}{\partial z}+\tilde{V}_{z} \frac{\partial v_{\theta}}{\partial z} \\
& =-\frac{1}{r} \frac{\partial p}{\partial \theta}+\frac{1}{R e}\left(\frac{\partial}{\partial r}\left(\frac{1}{r} \frac{\partial}{\partial r}\left(r v_{\theta}\right)\right)+\frac{1}{r^{2}} \frac{\partial^{2} v_{\theta}}{\partial \theta^{2}}+\frac{2}{r^{2}} \frac{\partial v_{r}}{\partial \theta}+\frac{\partial^{2} v_{\theta}}{\partial z^{2}}\right) \\
& \frac{\partial v_{z}}{\partial t}+v_{r} \frac{\partial \tilde{V}_{z}}{\partial r}+\tilde{V}_{r} \frac{\partial v_{z}}{\partial r}+\frac{v_{\theta}}{r} \frac{\partial \tilde{V}_{z}}{\partial \theta}+\frac{\tilde{V}_{\theta}}{r} \frac{\partial v_{z}}{\partial \theta}+v_{z} \frac{\partial \tilde{V}_{z}}{\partial z}+\tilde{V}_{z} \frac{\partial v_{z}}{\partial z} \\
& =-\frac{\partial p}{\partial z}+\frac{1}{R e}\left(\frac{1}{r} \frac{\partial}{\partial r}\left(r \frac{\partial v_{z}}{\partial r}\right)+\frac{1}{r^{2}} \frac{\partial^{2} v_{z}}{\partial \theta^{2}}+\frac{\partial^{2} v_{z}}{\partial z^{2}}\right)
\end{aligned}
$$

\subsection{Prototype flows}

So far, the domain on which the Navier-Stokes equations are defined has not entered the picture. In this tutorial, the focus is on two prototype flows that have been studied quite extensively in the literature: (1) channel flow, which is the flow contained between two paralel plates; and (2) pipe flow, which is the flow contained in the interior of a cylinder with circular cross section. The particularly simple geometries of these flows make the problems mathematically tractable, as well as numerically feasible. In addition, the pipe is encountered frequently in practice, so understanding how to control this flow is of great importance in engineering applications. 


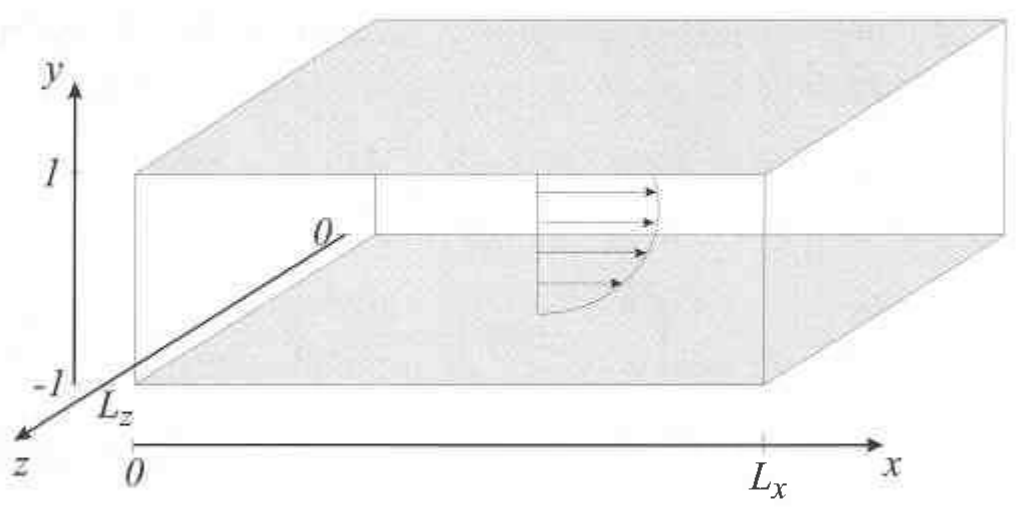

Figure 3. Geometry of the 3D channel flow.

2.6.1. $3 D$ channel flow The domain in this case is the box $\Omega=\left\{(x, y, z) \in\left[0, L_{x}\right) \times\right.$ $\left.(-1,1) \times\left[0, L_{z}\right)\right\}$. The flow problem is completed by specifying boundary conditions on the boundary of the box. In the streamwise $(x)$ and spanwise $(z)$ directions, we use periodic boundary conditions. That is, we equate the flow quantitites at $x=0$ and $x=L_{x}$, and at $z=0$ and $z=L_{z}$. At the walls, the boundary conditions will eventually be specified by the result of some boundary control design, but for the time being, we select no-slip boundary conditions, i.e. $U=V=W=0$ at the walls $(y= \pm 1)$. Given the boundary conditions, we are now in the position to solve equations (12)-(15) for a steady-state solution. In solving the Navier-Stokes equations for a steady-state solution in channel flow with no-slip boundary conditions at the walls, we assume that the velocity field is independent of the streamwise and spanwise directions. Thus, setting the time derivatives and the spatial derivatives with respect to $x$ and $z$ in equations (12)-(15) to zero, we get

$$
\begin{gathered}
\frac{\partial V}{\partial y}=0 \\
V \frac{\partial U}{\partial y}=-\frac{\partial P}{\partial x}+\frac{1}{R e} \frac{\partial^{2} U}{\partial y^{2}} \\
V \frac{\partial V}{\partial y}=-\frac{\partial P}{\partial y}+\frac{1}{R e} \frac{\partial^{2} V}{\partial y^{2}} \\
V \frac{\partial W}{\partial y}=-\frac{\partial P}{\partial z}+\frac{1}{R e} \frac{\partial^{2} W}{\partial y^{2}}
\end{gathered}
$$

From equation (41) we have that $V=$ constant, and since we are employing no-slip boundary conditions, we obtain $V=0$. So, from equations (42)-(44) we have that

$$
\begin{aligned}
& \frac{\partial P}{\partial x}=\frac{1}{R e} \frac{\partial^{2} U}{\partial y^{2}} \\
& \frac{\partial P}{\partial y}=0 \\
& \frac{\partial P}{\partial z}=\frac{1}{R e} \frac{\partial^{2} W}{\partial y^{2}}
\end{aligned}
$$


From equation (46), we have that $P=P(x, z)$, and since $U=U(y)$ by assumption, we get

$$
\begin{aligned}
& \frac{\partial P}{\partial x}=c_{1}=\frac{1}{R e} \frac{\partial^{2} U}{\partial y^{2}} \\
& \frac{\partial P}{\partial z}=c_{2}=\frac{1}{R e} \frac{\partial^{2} W}{\partial y^{2}}
\end{aligned}
$$

where $c_{1}$ and $c_{2}$ are constants. Thus, we can solve for each side of equations (48) and (49) seperately, to get

$$
P=c_{1} x+c_{2} z+c_{3}
$$

where $c_{3}$ is a constant, and

$$
\begin{gathered}
U(y)=\frac{c_{1} \operatorname{Re}}{2} y^{2}+c_{4} y+c_{5} \\
W(y)=\frac{c_{2} \operatorname{Re}}{2} y^{2}+c_{6} y+c_{7}
\end{gathered}
$$

where $c_{4}, c_{5}, c_{6}$ and $c_{7}$ are constants. Since only the gradient of $P$ enters the NavierStokes equations, $c_{3}$ may be arbitrarily chosen, so we set $c_{3}=0$. By employing no slip boundary conditions we obtain the set of equations

$$
\begin{aligned}
& \frac{c_{1} R e}{2}-c_{4}+c_{5}=0 \\
& \frac{c_{1} R e}{2}+c_{4}+c_{5}=0 \\
& \frac{c_{2} R e}{2}-c_{6}+c_{7}=0 \\
& \frac{c_{2} R e}{2}+c_{6}+c_{7}=0
\end{aligned}
$$

so that

$$
\begin{aligned}
& c_{4}=c_{6}=0 \\
& c_{5}=-\frac{c_{1} R e}{2} \\
& c_{7}=-\frac{c_{2} R e}{2}
\end{aligned}
$$

Without loss of generality we select the direction of flow along the positive $x$-axis, and the center velocity, $U(0)=1$. We get

$$
c_{1}=-\frac{2}{R e}, c_{2}=0, c_{5}=1, \text { and } c_{7}=0
$$




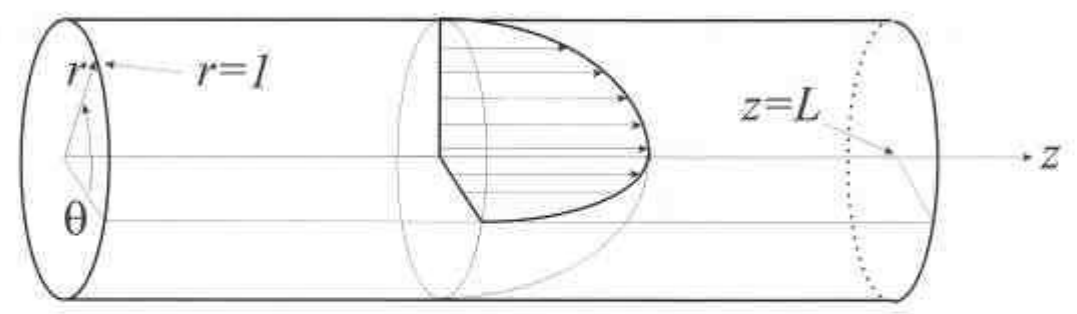

Figure 4. Geometry of the pipe flow.

So we have the steady state solution

$$
(\tilde{U}, \tilde{V}, \tilde{W}, \tilde{P})=\left(1-y^{2}, 0,0,-\frac{2}{R e} x\right)
$$

Inserting equation (50) into the perturbation equations equations (25)--(28) yields

$$
\begin{gathered}
\frac{\partial u}{\partial x}+\frac{\partial v}{\partial y}+\frac{\partial w}{\partial z}=0 \\
\frac{\partial u}{\partial t}+u \frac{\partial u}{\partial x}+\tilde{U} \frac{\partial u}{\partial x}+v \frac{\partial u}{\partial y}+v \frac{\partial \tilde{U}}{\partial y}+w \frac{\partial u}{\partial z}=-\frac{\partial p}{\partial x}+\frac{1}{R e}\left(\frac{\partial^{2} u}{\partial x^{2}}+\frac{\partial^{2} u}{\partial y^{2}}+\frac{\partial^{2} u}{\partial z^{2}}\right) \\
\frac{\partial v}{\partial t}+u \frac{\partial v}{\partial x}+\tilde{U} \frac{\partial v}{\partial x}+v \frac{\partial v}{\partial y}+w \frac{\partial v}{\partial z}=-\frac{\partial p}{\partial y}+\frac{1}{R e}\left(\frac{\partial^{2} v}{\partial x^{2}}+\frac{\partial^{2} v}{\partial y^{2}}+\frac{\partial^{2} v}{\partial z^{2}}\right) \\
\frac{\partial w}{\partial t}+u \frac{\partial w}{\partial x}+\tilde{U} \frac{\partial w}{\partial x}+v \frac{\partial w}{\partial y}+w \frac{\partial w}{\partial z}=-\frac{\partial p}{\partial z}+\frac{1}{R e}\left(\frac{\partial^{2} w}{\partial x^{2}}+\frac{\partial^{2} w}{\partial y^{2}}+\frac{\partial^{2} w}{\partial z^{2}}\right)
\end{gathered}
$$

The linearized equations around the steady state solution equation (50) are

$$
\begin{aligned}
& \frac{\partial u}{\partial x}+\frac{\partial v}{\partial y}+\frac{\partial w}{\partial z}=0 \\
& \frac{\partial u}{\partial t}+\tilde{U} \frac{\partial u}{\partial x}+v \frac{\partial \tilde{U}}{\partial y}=-\frac{\partial p}{\partial x}+\frac{1}{R e}\left(\frac{\partial^{2} u}{\partial x^{2}}+\frac{\partial^{2} u}{\partial y^{2}}+\frac{\partial^{2} u}{\partial z^{2}}\right) \\
& \frac{\partial v}{\partial t}+\tilde{U} \frac{\partial v}{\partial x}=-\frac{\partial p}{\partial y}+\frac{1}{R e}\left(\frac{\partial^{2} v}{\partial x^{2}}+\frac{\partial^{2} v}{\partial y^{2}}+\frac{\partial^{2} v}{\partial z^{2}}\right) \\
& \frac{\partial w}{\partial t}+\tilde{U} \frac{\partial w}{\partial x}=-\frac{\partial p}{\partial z}+\frac{1}{R e}\left(\frac{\partial^{2} w}{\partial x^{2}}+\frac{\partial^{2} w}{\partial y^{2}}+\frac{\partial^{2} w}{\partial z^{2}}\right)
\end{aligned}
$$

Choosing the channel half width (which is 1) as the characteristic length, and the center velocity (which is 1) as the characteristic velocity, the Reynolds number is simply $R e=\rho / \mu$. 
2.6.2. $3 D$ pipe flow The domain in this case is the cylinder $\Omega=\{(r, \theta, z) \in[0,1) \times$ $[0,2 \pi) \times[0, L)\}$. In the angular $(\theta)$ direction the boundary conditions are clearly periodic. In the streamwise $(z)$ direction, we alse use periodic boundary conditions. That is, we equate the flow quantitites at $\theta=0$ and $\theta=2 \pi$, and at $z=0$ and $z=L$. In the radial direction $(r)$ we impose the boundary conditions that the velocity be finite at $r=0$, and no-slip at the wall $(r=1)$. We are now in the position to solve equations (16)-(19) for a steady-state solution, which we assume is independent of the angular $(\theta)$ and streamwise $(z)$ directions with $V_{r}=V_{\theta}=0$. Thus, we get

$$
\begin{aligned}
0 & =\frac{\partial P}{\partial r} \\
0 & =\frac{\partial P}{\partial \theta} \\
\frac{\partial P}{\partial z} & =\frac{1}{\operatorname{Re}} \frac{1}{r} \frac{\partial}{\partial r}\left(r \frac{\partial V_{z}}{\partial r}\right)
\end{aligned}
$$

From equations (59) and (60) we have that $P=P(z)$, and since $V_{z}=V_{z}(r)$ by assumption, we get

$$
\frac{\partial P}{\partial z}=-c=\frac{1}{R e} \frac{1}{r} \frac{\partial}{\partial r}\left(r \frac{\partial V_{z}}{\partial r}\right)
$$

where $c$ is a constant. The left hand side of equation (62) can be integrated to obtain

$$
P=-c z+c_{1}
$$

and the right hand side of equation (62) is separable, so we can solve it to obtain

$$
V_{z}(r)=-\frac{c R e}{4} r^{2}+c_{2} \ln r+c_{3}
$$

Imposing the boundary conditions in the radial direction, yields

$$
c_{2}=0 \text { and } c_{3}=\frac{c R e}{4}
$$

and without loss of generality we select the center velocity, $V_{z}(0)=1$, so that $c=(4 / R e)$. Thus, we have the steady state solution

$$
\left(\tilde{V}_{r}, \tilde{V}_{\theta}, \tilde{V}_{z}, \tilde{P}\right)=\left(0,0,1-r^{2},-\frac{4}{R e} z\right)
$$

The perturbation equations are

$$
\begin{aligned}
& \frac{1}{r} \frac{\partial}{\partial r}\left(r v_{r}\right)+\frac{1}{r} \frac{\partial v_{\theta}}{\partial \theta}+\frac{\partial v_{z}}{\partial z}=0 \\
& \frac{\partial v_{r}}{\partial t}+v_{r} \frac{\partial v_{r}}{\partial r}+\frac{v_{\theta}}{r} \frac{\partial v_{r}}{\partial \theta}-\frac{v_{\theta}^{2}}{r}+v_{z} \frac{\partial v_{r}}{\partial z}+\tilde{V}_{z} \frac{\partial v_{r}}{\partial z}=-\frac{\partial p}{\partial r}+\frac{1}{\operatorname{Re}}\left(\frac{\partial}{\partial r}\left(\frac{1}{r} \frac{\partial}{\partial r}\left(r v_{r}\right)\right)\right. \\
& \left.+\frac{1}{r^{2}} \frac{\partial^{2} v_{r}}{\partial \theta^{2}}-\frac{2}{r^{2}} \frac{\partial v_{\theta}}{\partial \theta}+\frac{\partial^{2} v_{r}}{\partial z^{2}}\right)
\end{aligned}
$$




$$
\begin{aligned}
& \frac{\partial v_{\theta}}{\partial t}+v_{r} \frac{\partial v_{\theta}}{\partial r}+\frac{v_{\theta}}{r} \frac{\partial v_{\theta}}{\partial \theta}+\frac{v_{r} v_{\theta}}{r}+v_{z} \frac{\partial v_{\theta}}{\partial z}+\tilde{V}_{z} \frac{\partial v_{\theta}}{\partial z}=-\frac{1}{r} \frac{\partial p}{\partial \theta}+\frac{1}{R e}\left(\frac{\partial}{\partial r}\left(\frac{1}{r} \frac{\partial}{\partial r}\left(r v_{\theta}\right)\right)\right. \\
& \left.+\frac{1}{r^{2}} \frac{\partial^{2} v_{\theta}}{\partial \theta^{2}}+\frac{2}{r^{2}} \frac{\partial v_{r}}{\partial \theta}+\frac{\partial^{2} v_{\theta}}{\partial z^{2}}\right) \\
& \frac{\partial v_{z}}{\partial t}+v_{r} \frac{\partial v_{z}}{\partial r}+v_{r} \frac{\partial \tilde{V}_{z}}{\partial r}+\frac{v_{\theta}}{r} \frac{\partial v_{z}}{\partial \theta}+v_{z} \frac{\partial v_{z}}{\partial z}+\tilde{V}_{z} \frac{\partial v_{z}}{\partial z}=-\frac{\partial p}{\partial z}+\frac{1}{\operatorname{Re}}\left(\frac{1}{r} \frac{\partial}{\partial r}\left(r \frac{\partial v_{z}}{\partial r}\right)\right. \\
& \left.+\frac{1}{r^{2}} \frac{\partial^{2} v_{z}}{\partial \theta^{2}}+\frac{\partial^{2} v_{z}}{\partial z^{2}}\right)
\end{aligned}
$$

and the linearized equations around the steady state solution equation (63) are

$$
\begin{gathered}
\frac{1}{r} \frac{\partial}{\partial r}\left(r v_{r}\right)+\frac{1}{r} \frac{\partial v_{\theta}}{\partial \theta}+\frac{\partial v_{z}}{\partial z}=0 \\
\frac{\partial v_{r}}{\partial t}+\tilde{V}_{z} \frac{\partial v_{r}}{\partial z}=-\frac{\partial p}{\partial r}+\frac{1}{R e}\left(\frac{\partial}{\partial r}\left(\frac{1}{r} \frac{\partial}{\partial r}\left(r v_{r}\right)\right)+\frac{1}{r^{2}} \frac{\partial^{2} v_{r}}{\partial \theta^{2}}-\frac{2}{r^{2}} \frac{\partial v_{\theta}}{\partial \theta}+\frac{\partial^{2} v_{r}}{\partial z^{2}}\right) \\
\frac{\partial v_{\theta}}{\partial t}+\tilde{V}_{z} \frac{\partial v_{\theta}}{\partial z}=-\frac{1}{r} \frac{\partial p}{\partial \theta}+\frac{1}{R e}\left(\frac{\partial}{\partial r}\left(\frac{1}{r} \frac{\partial}{\partial r}\left(r v_{\theta}\right)\right)+\frac{1}{r^{2}} \frac{\partial^{2} v_{\theta}}{\partial \theta^{2}}+\frac{2}{r^{2}} \frac{\partial v_{r}}{\partial \theta}+\frac{\partial^{2} v_{\theta}}{\partial z^{2}}\right) \\
\frac{\partial v_{z}}{\partial t}+v_{r} \frac{\partial \tilde{V}_{z}}{\partial r}+\tilde{V}_{z} \frac{\partial v_{z}}{\partial z}=-\frac{\partial p}{\partial z}+\frac{1}{R e}\left(\frac{1}{r} \frac{\partial}{\partial r}\left(r \frac{\partial v_{z}}{\partial r}\right)+\frac{1}{r^{2}} \frac{\partial^{2} v_{z}}{\partial \theta^{2}}+\frac{\partial^{2} v_{z}}{\partial z^{2}}\right)
\end{gathered}
$$

Choosing the pipe radius (which is 1) as the characteristic length, and the center velocity (which is 1 ) as the characteristic velocity, the Reynolds number is simply $\operatorname{Re}=\rho / \mu$.

2.6.3. $2 \mathrm{D}$ channel/pipe flow In $2 \mathrm{D}$, the channel and pipe flows coincide. The domain is in this case $\Omega=\{(x, y) \in[0, L) \times(-1,1)\}$, and periodic boundary conditions are employed in the streamwise $(x)$ direction. With no-slip boundary conditions at the wall, we have the steady-state solution

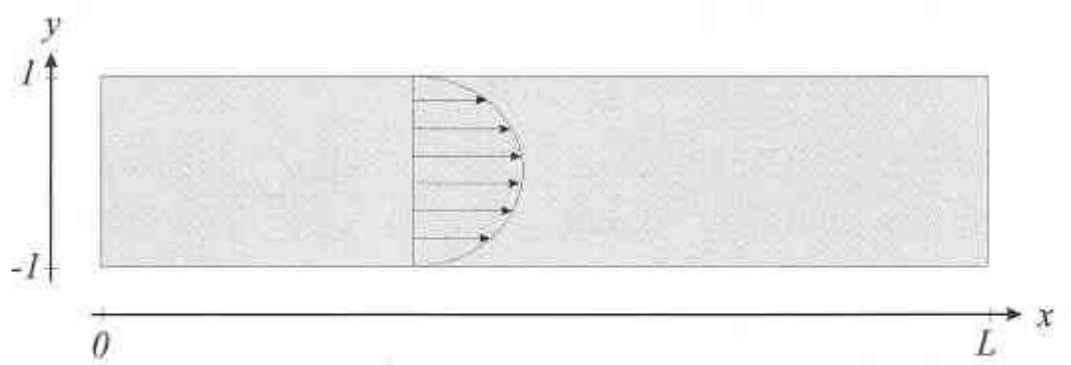

Figure 5. Geometry of the 2D channel flow. 


$$
(\tilde{U}, \tilde{V}, \tilde{P})=\left(1-y^{2}, 0,-\frac{2}{\operatorname{Re}} x\right)
$$

and the perturbation equations become

$$
\begin{gathered}
\frac{\partial u}{\partial x}+\frac{\partial v}{\partial y}=0 \\
\frac{\partial u}{\partial t}+u \frac{\partial u}{\partial x}+\tilde{U} \frac{\partial u}{\partial x}+v \frac{\partial u}{\partial y}+v \frac{\partial \tilde{U}}{\partial y}=-\frac{\partial p}{\partial x}+\frac{1}{\operatorname{Re}\left(\frac{\partial^{2} u}{\partial x^{2}}+\frac{\partial^{2} u}{\partial y^{2}}\right)} \\
\frac{\partial v}{\partial t}+u \frac{\partial v}{\partial x}+\tilde{U} \frac{\partial v}{\partial x}+v \frac{\partial v}{\partial y}=-\frac{\partial p}{\partial y}+\frac{1}{R e}\left(\frac{\partial^{2} v}{\partial x^{2}}+\frac{\partial^{2} v}{\partial y^{2}}\right)
\end{gathered}
$$

The linearized equations around the steady state solution equation (72) are

$$
\begin{aligned}
& \frac{\partial u}{\partial x}+\frac{\partial v}{\partial y}=0 \\
& \frac{\partial u}{\partial t}+\tilde{U} \frac{\partial u}{\partial x}+v \frac{\partial \tilde{U}}{\partial y}=-\frac{\partial p}{\partial x}+\frac{1}{R e}\left(\frac{\partial^{2} u}{\partial x^{2}}+\frac{\partial^{2} u}{\partial y^{2}}\right) \\
& \frac{\partial v}{\partial t}+\tilde{U} \frac{\partial v}{\partial x}=-\frac{\partial p}{\partial y}+\frac{1}{R e}\left(\frac{\partial^{2} v}{\partial x^{2}}+\frac{\partial^{2} v}{\partial y^{2}}\right)
\end{aligned}
$$

Choosing the channel half width (which is 1) as the characteristic length, and the center velocity (which is 1 ) as the characteristic velocity, the Reynolds number is simply $R e=\rho / \mu$.

\subsection{Spatial discretization}

For simulation purposes, as well as some approaches to control design, one needs to discretize the equations of motion both spatially and temporally. For a general treatment of the discretization of the Navier-Stokes equations, see Ferziger and Perić (1999). In this section, the treatment of this subject is restricted to the methods used in the design of controllers in the next chapter. These are so-called spectral methods (see Canuto et al. (1988) for an in-depth treatment of spectral methods applied to partial differential equations, including the two methods outlined below; the FourierGalerkin method, and the Chebyshev collocation method), which lend themselves particularly well suited for the prototype flows that were described in the previous section.

2.7.1. Spectral methods Consider the cvolution equation

$$
\frac{\partial u}{\partial t}=A(u)
$$

with the initial condition

$$
u(x, 0)-u_{0}(x)
$$


where $u \in \mathscr{X}$ is the solution sought, and $A$ is an operator containing the spatial derivatives of $u$. The equation is defined on the spatial domain $\Omega$, which we will assume is one-dimensional for simplicity, that is, $x \in \Omega \subset \mathbb{R} . \mathscr{X}$ is a Hilbert space with scalar product

$$
(u, v)_{\mathscr{X}}=\int_{\Omega} u \bar{v} d \Omega
$$

and norm

$$
\|u\|_{x}=(u, u)^{1 / 2}
$$

The bar $(\bar{v})$ denotes complex conjugation. In order to complete the problem, appropriate boundary conditions must be supplied. Now, consider a series expansion for the solution $u$, that is

$$
u^{*}(x, t)=\sum_{k} a_{k}(t) \phi_{k}(x)
$$

where the $\phi_{k}$ are called the trial functions, and the $a_{k}$ are called the expansion coefficients. The $\phi_{k}$ are assumed to constitute a complete basis for $\mathscr{X}$, so that the series equation (80) converges to $u$ in $\mathscr{X}$ as $k \rightarrow \infty$. The approximation of equation (80), of order $N$, is defined as the truncated series

$$
u^{N}(x, t)=\sum_{k \in I} a_{k}(t) \phi_{k}(x)
$$

where $I$ is a finite set of indices. Thus, $u^{N} \in \mathscr{X}^{N}=\operatorname{span}\left\{\phi_{k} \mid k \in I\right\} \subset \mathscr{X}$. Since $u^{N}$ is an approximate solution of equation (79), the residual

$$
R\left(u^{N}\right)=\frac{\partial u^{N}}{\partial t}-A\left(u^{N}\right)
$$

will in general not vanish everywhere, but its projection onto the span of a set of test functions, $\mathscr{Y}^{N}=\operatorname{span}\left\{\psi_{j} \mid j \in I\right\}$, is required to be zero. That is,

$$
\left(\frac{\partial u^{N}}{\partial t}-A\left(u^{N}\right), \psi_{j}\right)_{\mathscr{X}}=\int_{\Omega}\left(\frac{\partial u^{N}}{\partial t}-A\left(u^{N}\right)\right) \bar{\psi}_{j} d \Omega=0, j \in I
$$

Equation (81) results in $N$ ordinary differential equation for the determination of the expansion coefficients $a_{k}$.

2.7.2. The Fourier-Galerkin method The Galerkin method is characterized by the fact that the trial functions and the test functions are the same, and that each test function satisfies the boundary conditions. When the boundary conditions are periodic with period $L$, that is $\Omega=[0, L)$, a natural choice for the trial functions is

$$
\phi_{k}=\psi_{k}=e^{i k(2 \pi / L) x}, k=-N / 2, \ldots, N / 2
$$

With this choice of test and trial functions, equation (81) is called the FourierGalerkin method. The $\phi_{k}$ constitute an orthogonal basis for $\mathscr{X}^{N}$, since

$$
\left(\phi_{j}, \phi_{k}\right)_{\mathscr{X}}=\int_{0}^{L} \phi_{j} \bar{\phi}_{k} d x=\int_{0}^{L} e^{i(j-k)(2 \pi / L) x} d x=\left\{\begin{array}{l}
L, j=k \\
0, j \neq k
\end{array}\right.
$$


The main advantage of using trigonometric polynomials is the simplicity and accuracy in calculating the derivatives

$$
\frac{d^{m}}{d x^{m}} \phi_{k}=\left(\frac{2 \pi i k}{L}\right)^{m} \phi_{k}
$$

As an example, assume that the operator $A$ is linear, and contains spatial derivatives up to order $M$. In view of equation (82), the operator $A$ acting on $\phi_{k}$ can be represented as the finite sum

$$
A\left(\phi_{k}\right)=\sum_{m=0}^{M} c_{m k} \phi_{k}
$$

where the $c_{m k}$ are complex constants. From equation (81), keeping in mind that $A$ is linear and that the scalar product is bilinear, we get

$$
\begin{aligned}
& \left(\frac{\partial}{\partial t}\left(\sum_{k=-N / 2}^{N / 2} a_{k}(t) \phi_{k}\right)-A\left(\sum_{k=-N / 2}^{N / 2} a_{k}(t) \phi_{k}\right), \phi_{j}\right)_{X} \\
& =\sum_{k=-N / 2}^{N / 2}\left(\frac{\partial a_{k}(t)}{\partial t}-a_{k}(t) \sum_{m=0}^{M} c_{m k}\right)\left(\phi_{k}, \phi_{j}\right)_{\mathscr{X}} \\
& =L\left(\frac{\partial a_{j}(t)}{\partial t}-a_{j}(t) \sum_{m=0}^{M} c_{m j}\right)
\end{aligned}
$$

Thus, the finite set of ordinary differential equations

$$
\frac{d a_{k}(t)}{d t}-a_{k}(t) \sum_{m=0}^{M} c_{m k}=0, \text { for } k=-N / 2, \ldots, N / 2
$$

determines the expansion coefficients $a_{k}$. The initial condition is obtained by the usual relation (Fourier transform)

$$
a_{k}(0)=\frac{1}{L} \int_{0}^{L} u_{0}(x) e^{-i k(2 \pi / L) x} d x, k=-N / 2, \ldots, N / 2
$$

2.7.3. The Chebyshev collocation method The collocation method is characterized by the fact that the test functions are shifted Dirac functions, $\psi_{j}(x)=\delta\left(x-x_{j}\right)$, which are defined by

$$
\int_{\Omega} \delta\left(x-x_{j}\right) f d x=f\left(x_{j}\right)
$$

Thus, equation (81) reduces to

$$
\begin{aligned}
\left(\frac{\partial u^{N}}{\partial t}-A\left(u^{N}\right), \psi_{j}\right)_{\mathscr{X}} & =\int_{\Omega}\left(\frac{\partial u^{N}}{\partial t}-A\left(u^{N}\right)\right) \bar{\psi}_{j} d \Omega \\
& =\left(\frac{\partial u^{N}\left(x_{j}\right)}{\partial t}-A\left(u^{N}\left(x_{j}\right)\right)\right)=0, j=1,2,3, \ldots, N-1
\end{aligned}
$$


The boundary conditions are taken to be $u^{N}(-1, t)=u^{N}\left(x_{0}, t\right)$, and $u^{N}(1, t)=u^{N}\left(x_{N}, t\right)$, and the initial condition is $u^{N}\left(x_{j}, 0\right)=u\left(x_{j}, 0\right)$, for $j=0,1,2, \ldots, N$. Unlike the Galerkin method, which is implemented in terms of the expansion coefficients, $a_{k}$, the collocation method is implemented in terms of $u$ at the collocation points, $x_{j}$. The expansion coefficients are used in the differentiation of $u^{N}$. In the Chebyshev collocation method, the trial functions are Chebyshev polynomials (or a linear combination of Chebyshev polynomials), which are defined on $\Omega=[-1,1]$ as

$$
\phi_{k}(x)=\cos (k \theta), \theta=\cos ^{-1}(x) \text { for } k=0,1,2,3, \ldots
$$

The Chebyshev expansion of $u$ is

$$
u^{*}(x)=\sum_{k=0}^{\infty} a_{k} \phi_{k}(x), a_{k}=\frac{2}{\pi c_{k}} \int_{-1}^{1} u(x) \phi_{k}(x) w(x) d x
$$

where

$$
\begin{gathered}
c_{k}=\left\{\begin{array}{l}
2, k=0 \\
1, k>0
\end{array}\right. \\
w(x)=\frac{1}{\sqrt{1-x^{2}}}
\end{gathered}
$$

The derivative of equation (84) is

$$
\frac{d u(x)}{d x}=\sum_{k=0}^{\infty} a_{k} \frac{d \phi_{k}(x)}{d x}
$$

which is computed most efficiently by deriving a recursive formula. For $k \geqslant 1$, we have that

$$
\begin{aligned}
& \frac{d \phi_{k-1}(x)}{d x}=-(k-1) \sin ((k-1) \theta) \frac{d \theta}{d x} \\
& \frac{d \phi_{k+1}(x)}{d x}=-(k+1) \sin ((k+1) \theta) \frac{d \theta}{d x}
\end{aligned}
$$

which, by standard trigonometric relationships, gives

$$
\frac{1}{k+1} \frac{d \phi_{k+1}(x)}{d x}-\frac{1}{k-1} \frac{d \phi_{k-1}(x)}{d x}=-2 \cos (k \theta) \sin (\theta) \frac{d \theta}{d x}
$$

Since

$$
\frac{d \theta}{d x}=-\frac{1}{\sqrt{1-x^{2}}}=-\frac{1}{\sin (\theta)}
$$

we obtain

$$
\frac{1}{k+1} \frac{d \phi_{k+1}(x)}{d x}-\frac{1}{k-1} \frac{d \phi_{k-1}(x)}{d x}=2 \phi_{k}(x)
$$

Thus, the derivative of the trial functions obeys the recursive formula

$$
\frac{d \phi_{k}(x)}{d x}=\frac{k}{k-2} \frac{d \phi_{k-2}(x)}{d x}+2 k \phi_{k-1}(x), k \geqslant 2
$$


Repeated use of equation (85) yields

$$
\begin{gathered}
\frac{d \phi_{k}(x)}{d x}=2 k\left(\phi_{k-1}(x)+\phi_{k-3}(x)+\phi_{k-5}(x)+\ldots+\frac{1}{2-l} \phi_{l}(x)\right), \\
k \geqslant 2, l=\left\{\begin{array}{l}
0 \text { for } k \text { odd } \\
1 \text { for } k \text { even }
\end{array}\right.
\end{gathered}
$$

From $d \phi_{0}(x) / d x=0, d \phi_{1}(x) / d x=\phi_{0}(x)$, we see that equation (86) is valid for all $k \geqslant 0$. It follows from equation (86) that the expansion coefficients for $d u / d x$, denoted $a_{k}^{d}$, are given as

$$
\begin{aligned}
& a_{0}^{d}=\sum_{\substack{m=1 \\
m \text { odd }}}^{\infty} m a_{m} \\
& a_{k}^{d}=2 \sum_{\substack{m=k+1 \\
m+k \text { odd }}}^{\infty} m a_{m}, k \geqslant 1
\end{aligned}
$$

The accuracy of the approximation is highly dependent on the choice of collocation points, and a common choice is (Chebyshev-Gauss-Lobatto)

$$
x_{j}=-\cos \frac{\pi j}{N}, j=0,1,2, \ldots, N
$$

In the discrete case, the expansion of the approximation is the truncated series

$$
u(x)=\sum_{k=0}^{N} a_{k} \phi_{k}(x)
$$

with the discrete expansion coefficients

$$
a_{k}=\frac{1}{\gamma_{k}} \sum_{j=0}^{N} u\left(x_{j}\right) \phi_{k}\left(x_{j}\right) w_{j}
$$

where

$$
\gamma_{k}=\left\{\begin{array}{ll}
\pi, & \text { for } k=0, N \\
\frac{\pi}{2}, & \text { for } 0<k<N
\end{array} \quad \text { and } \quad w_{j}= \begin{cases}\frac{\pi}{2 N}, & \text { for } j=0, N \\
\frac{\pi}{N}, & \text { for } 0<j<N\end{cases}\right.
$$

Equations (87) (88) provide a recursive formula for the calculation of the derivatives in Chebyshev space

$$
\begin{aligned}
a_{k \geqslant N}^{d} & =0 \\
a_{k}^{d} & =2 \sum_{\substack{m=k+1 \\
m+k \text { odd }}}^{N} m a_{m}=2(k+1) a_{k+1}+a_{k+2}^{d}, k \geqslant 1
\end{aligned}
$$




$$
a_{0}^{d}=a_{1}+\sum_{\substack{m=3 \\ m \text { odd }}}^{\infty} m a_{m}=a_{1}+\frac{1}{2} a_{2}^{d}
$$

As for the Fourier-Galerkin method, the result is a finite set of ordinary differential equations, which are difficult to write in this case. The discrete derivative may also be expressed as a matrix multiplication, that is

$$
\frac{d u^{N}\left(x_{k}\right)}{d x}=\sum_{j=0}^{N}\left(\mathscr{D}_{N}\right)_{k j} u^{N}\left(x_{j}\right)
$$

where $\mathscr{D}_{N}$ is the $N+1 \times N+1$ matrix

$$
\left(\mathscr{I}_{N}\right)_{k j} \triangleq d_{k j}=\left\{\begin{array}{cc}
\frac{\bar{c}_{k}}{\bar{c}_{j} \frac{(-1)^{k+j}}{x_{k}-x_{j}}} & k \neq j \\
-\frac{x_{j}}{2\left(1-x_{j}^{2}\right)} & 1 \leqslant k=j \leqslant N-1 \\
\frac{2 N^{2}+1}{6} & k=j=1 \\
-\frac{2 N^{2}+1}{6} & k=j=N
\end{array}\right.
$$

Computationally, the Chebyshev transform is superior to matrix multiplication for computing the derivative when $N>\sim 20$.

\section{Stability Properties of Flows}

The steady-state solutions, or equilibrium velocity profiles, obtained for the prototype flows of Section 2.6, are parabolic in shape in the streamwise direction, and zero in the other directions. Thus, the flows consist of paralel layers of fluid moving in a very regular and deterministic way. These are examples of so-called laminar flows. For wall-bounded laminar flows, wall friction, or drag, is favorably low, and these flows are therefore target flows in drag reduction applications. Unfortunately, they are rarely stable. In fact, stability is assured at small Reynolds number, only.

An unstable flow is characterized by the fact that small perturbations from the equilibrium velocity profile will grow, and eventually cause the flow to transition to turbulent flow. Turbulent flow is characterized by small scale, appearently stochastic, velocity components, which lead to substantially higher drag than what is present in laminar flow. Being able to relaminarize a turbulent flow is therefore of great importance, and can be achieved in the prototype flows studied here, by stabilizing the parabolic equilibrium profile using boundary control. Boundary control implies specifying the flow field dynamically on the boundary of the domain, in this case on the channel or pipe walls, possibly based on values of flow variables taken at the boundary. In this work, we assume that there exist sensors that provide distributed flow information at the wall, and actuators that can set prescribed distributed velocities. The micromachining technology that emerged in the 1980s allows for the manufacturing of micron sized sensors and actuators that accomplish this task (see Ho and Tai (1996); Ho and Tai (1998) for reviews). 
The problem of stabilizing the parabolic equilibrium profile of the channel flow has been attacked from several different angles by a number of authors. Approaches range from discretizing the linearized Navier-Stokes equations and using the tools available for stabilizing finite-dimensional linear time invariant systems, to Lyapunov stability analysis of the full, nonlinear Navier-Stokes equations. In the following sections, these efforts are summarized.

\section{Linearization and Reduced Order Methods}

This section summarizes the efforts on stabilization of the discretized, linearized Navier-Stokes equations. Bringing the linearized Navier-Stokes equations in the form of a linear time invariant system constitute a major part of the work involved in these methods. Once the state-space model is constructed, any tool from linear control theory can be applied in a fairly straight forward manner.

\subsection{D channel flow}

In a series of papers (Cortelezzi et al., 1998; Cortelezzi and Speyer, 1998; Joshi et al., 1997; Joshi et al., 1999), stabilization of a reduced order model of 2D channel flow by classical and optimal control techniques is considered. The shear $(\partial U / \partial y)$ at a single point on the lower wall is taken as measurement, and the rate of change in the intensity of fluid transpiration on the lower wall is the control input. The actuator applies blowing and suction of fluid distributed as a prescribed function of $x$ along the lower wall. The control system setup is shown schematically in Figure 6.

4.1.1. Reduced order model The point of departure for obtaining a reduced order model on state space form is the linearized Navier-Stokes equation for 2D channel flow, equations (76) and (77)-(78). Due to equation (76) there exists a single valued function $\psi(x, y, t)$ such that

$$
\begin{aligned}
& u(x, y, t)=\frac{\partial \psi}{\partial y}(x, y, t) \\
& v(x, y, t)=-\frac{\partial \psi}{\partial x}(x, y, t)
\end{aligned}
$$

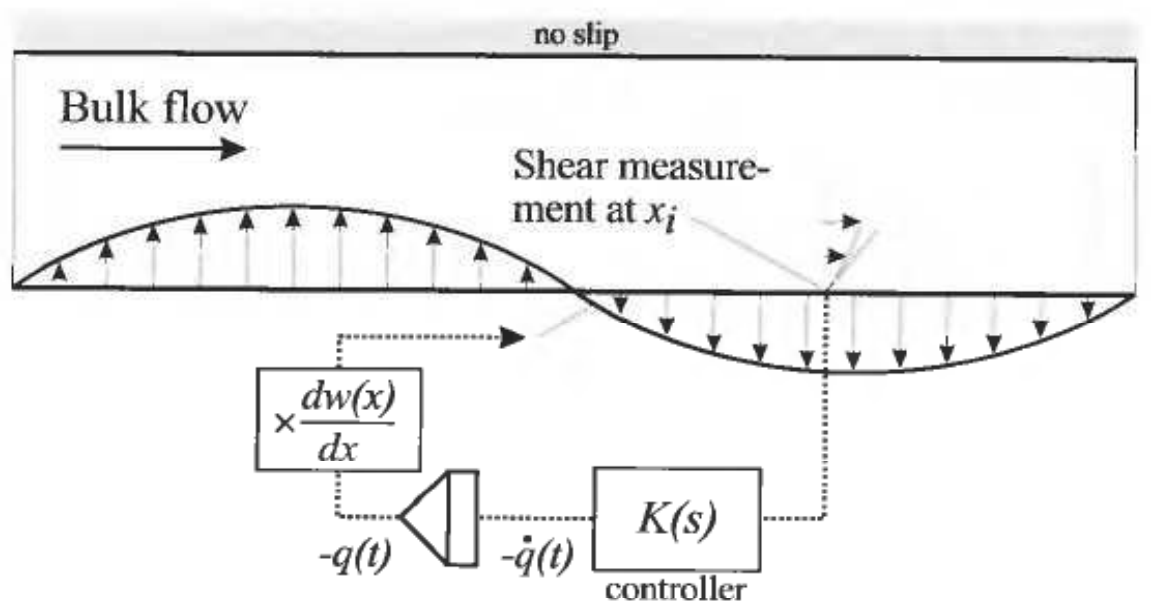

Figure 6. Control system configuration for controlling 2D channel flow by wall transpiration at the lower wall. 
The function $\psi$ is unique up to a constant, and is called the stream function due to the fact that contours of constant $\psi$ define streamlines of the flow. Inserting equations (91)-(92) into equations (77)-(78) yields

$$
\begin{array}{r}
\frac{\partial^{2} \psi}{\partial t \partial y}+\tilde{U} \frac{\partial^{2} \psi}{\partial y \partial x}-\frac{\partial \tilde{U}}{\partial y} \frac{\partial \psi}{\partial x}+\frac{\partial p}{\partial x}-\frac{1}{R e}\left(\frac{\partial^{3} \psi}{\partial y \partial x^{2}}+\frac{\partial^{3} \psi}{\partial y^{3}}\right)=0 \\
-\frac{\partial^{2} \psi}{\partial t \partial x}-\tilde{U} \frac{\partial^{2} \psi}{\partial x^{2}}+\frac{\partial p}{\partial y}+\frac{1}{R e}\left(\frac{\partial^{3} \psi}{\partial x^{3}}+\frac{\partial^{3} \psi}{\partial x \partial y^{2}}\right)=0
\end{array}
$$

Taking the partial derivative of equation (93) with respect to $y$ and subtracting the partial derivative of equation (94) with respect to $x$ yield

$$
\frac{\partial^{3} \psi}{\partial t \partial y^{2}}+\frac{\partial^{3} \psi}{\partial t \partial x^{2}}+\tilde{U} \frac{\partial^{3} \psi}{\partial x \partial y^{2}}-\frac{\partial^{2} \tilde{U}}{\partial y^{2}} \frac{\partial \psi}{\partial x}+\tilde{U} \frac{\partial^{3} \psi}{\partial x^{3}}-\frac{1}{R e}\left(\frac{\partial^{4} \psi}{\partial x^{4}}+2 \frac{\partial^{4} \psi}{\partial x^{2} \partial y^{2}}+\frac{\partial^{4} \psi}{\partial y^{4}}\right)=0
$$

Suppose boundary control is applied by imposing a boundary condition on the lower wall of the form

$$
\psi(x, y=-1, t)=q(t) w(x) f(y=-1)
$$

while keeping no slip boundary conditions on the upper wall, that is

$$
\frac{\partial \psi}{\partial y}(x, y=1, t)=\frac{\partial \psi}{\partial x}(x, y=1, t)=0
$$

Restricting the boundary control to blowing and suction of fluid through the wall at normal angle to the wall, that is $u(x, y=-1, t)=(\partial \psi / \partial y)(x, y=-1, t)=0$, along with the no slip condition on the upper wall, restrict the function $f(y)$ in the following manner

$$
\begin{gathered}
\frac{\partial f}{\partial y}(y= \pm 1)=0 \\
f(y=1)=0
\end{gathered}
$$

As in Joshi et al. (1997), we will use

$$
f(y)=\frac{1}{2} y^{4}+\frac{1}{4} y^{3}-y^{2}-\frac{3}{4} y+1
$$

although there are many other choices satisfying equations (97)-(98). When implementing the boundary control law equation (96) in practice, the physical flow variables, $U$ and $V$, must be set such that the resulting stream function satisfies equation (96). In terms of the physical flow variables $U$ and $V$, the boundary control law is

$$
\begin{aligned}
& U(x, y= \pm 1, t)=0, V(x, y=1, t)=0 \\
& V(x, y=-1, t)=v(x, y=-1, t)=-\frac{\partial \psi}{\partial x}(x, y=-1, t)=-q(t) \frac{\partial w}{\partial x}(x) f(y=-1)
\end{aligned}
$$


In order to obtain homogeneous boundary conditions, we introduce the change of variables

$$
\phi(x, y, t) \triangleq \psi(x, y, t)-q(t) w(x) f(y)
$$

which substituted into equation (95) yields

$$
\begin{aligned}
\frac{\partial^{3} \phi}{\partial t \partial y^{2}} & +\frac{d q}{d t} w \frac{d^{2} f}{d y^{2}}+\frac{\partial^{3} \phi}{\partial t \partial x^{2}}+\frac{d q}{d t} \frac{d^{2} w}{d x^{2}} f+\tilde{U}\left(\frac{\partial^{3} \phi}{\partial x \partial y^{2}}+q \frac{d w}{d x} \frac{d^{2} f}{d y^{2}}\right) \\
& -\frac{d^{2} \tilde{U}}{d y^{2}}\left(\frac{\partial \phi}{\partial x}+q \frac{d w}{d x} f\right)+\tilde{U}\left(\frac{\partial^{3} \phi}{\partial x^{3}}+q \frac{d^{3} w}{d x^{3}} f\right) \\
& -\frac{1}{\operatorname{Re}}\left(\left(\frac{\partial^{4} \phi}{\partial x^{4}}+q \frac{d^{4} w}{d x^{4}} f\right)+2\left(\frac{\partial^{4} \phi}{\partial x^{2}} \partial y^{2}+q \frac{d^{2} w}{d x^{2}} \frac{d^{2} f}{d y^{2}}\right)+\left(\frac{\partial^{4} \phi}{\partial y^{4}}+q w \frac{d^{4} f}{d y^{4}}\right)\right)=0
\end{aligned}
$$

The boundary conditions in terms of $\phi$ are

$$
\begin{array}{r}
\phi(x, y= \pm 1, t)=0 \\
\frac{\partial \phi}{\partial y}(x, y= \pm 1, t)=0
\end{array}
$$

The streamwise component of shear at a single point at the lower wall is used as a measurement, that is

$$
z(t)=\frac{\partial u}{\partial y}\left(x_{i}, y=-1, t\right)
$$

In terms of $\phi$, we have

$$
z(t)=\frac{\partial^{2} \psi}{\partial y^{2}}\left(x_{i}, y=-1, t\right)=\frac{\partial^{2} \phi}{\partial y^{2}}\left(x_{i}, y=-1, t\right)+q(t) w\left(x_{i}\right) \frac{d^{2} f}{d y^{2}}(y=-1)
$$

A standard Fourier-Galerkin procedure is used to discretize the partial differential equation in the streamwise direction, and a Chebyshev collocation method is used in the wall-normal direction. Starting with the streamwise direction, we set

$$
\begin{gathered}
\phi(x, y, t)=\sum_{n=-N}^{N} a_{n}(y, t) P_{n}(x) \\
w(x)=\sum_{n=-N}^{N} w_{n} P_{n}(x)
\end{gathered}
$$

where

$$
P_{n}(x)=e^{i n(2 \pi / L) x}
$$

The partial derivatives of $\phi$ and $w$ with respect to $x$ up to order four are

$$
\frac{\partial \phi}{\partial x}(x, y, t)=\sum_{n=-N}^{N} \alpha_{n} i a_{n}(y, t) P_{n}(x) \quad \frac{d w}{d x}(x)=\sum_{n=-N}^{N} \alpha_{n} i w_{n} P_{n}(x)
$$




$$
\begin{array}{ll}
\frac{\partial^{2} \phi}{\partial x^{2}}(x, y, t)=-\sum_{n=-N}^{N} \alpha_{n}^{2} a_{n}(y, t) P_{n}(x) & \frac{d^{2} w}{d x^{2}}(x)=-\sum_{n=-N}^{N} \alpha_{n}^{2} w_{n} P_{n}(x) \\
\frac{\partial^{3} \phi}{\partial x^{3}}(x, y, t)=-\sum_{n=-N}^{N} \alpha_{n}^{3} i a_{n}(y, t) P_{n}(x) & \frac{d^{3} w}{d x^{3}}(x)=-\sum_{n=-N}^{N} \alpha_{n}^{3} i w_{n} P_{n}(x) \\
\frac{\partial^{4} \phi}{\partial x^{4}}(x, y, t)=\sum_{n=-N}^{N} \alpha_{n}^{4} a_{n}(y, t) P_{n}(x) & \frac{d^{4} w}{d x^{4}}(x)=\sum_{n=-N}^{N} \alpha_{n}^{4} w_{n} P_{n}(x)
\end{array}
$$

where $\alpha_{n}=2 \pi n / L$. The Gelerkin method provides the set of equations

$$
\begin{aligned}
\int_{0}^{L}\left\{\frac{\partial^{3} \phi}{\partial t \partial y^{2}}+\frac{d q}{d t} w \frac{d^{2} f}{d y^{2}}+\frac{\partial^{3} \phi}{\partial t \partial x^{2}}+\frac{d q}{d t} \frac{d^{2} w}{d x^{2}} f+\tilde{U}\left(\frac{\partial^{3} \phi}{\partial x \partial y^{2}}+q \frac{d w}{d x} \frac{d^{2} f}{d y^{2}}\right)\right. \\
-\frac{\partial^{2} \tilde{U}}{\partial y^{2}}\left(\frac{\partial \phi}{\partial x}+q \frac{d w}{d x} f\right)+\tilde{U}\left(\frac{\partial^{3} \phi}{\partial x^{3}}+q \frac{d^{3} w}{d x^{3}} f\right) \\
\left.-\frac{1}{\operatorname{Re}}\left(\frac{\partial^{4} \phi}{\partial x^{4}}+q \frac{d^{4} w}{d x^{4}} f+2\left(\frac{\partial^{4} \phi}{\partial x^{2}} \partial y^{2}+q \frac{d^{2} w}{d x^{2}} \frac{d^{2} f}{d y^{2}}\right)+\frac{\partial^{4} \phi}{\partial y^{4}}+q w \frac{d^{4} f}{d y^{4}}\right)\right\} \bar{P}_{k}(x) d x=0
\end{aligned}
$$

Inserting equations (101)-(102) and (104)-(107) into (108) yields

$$
\begin{aligned}
\int_{0}^{L} \sum_{n=-N}^{N}\{ & {\left[\frac{\partial^{3} a_{n}}{\partial t \partial y^{2}}+\frac{d q}{d t} \frac{d^{2} f}{d y^{2}} w_{n}-\alpha_{n}^{2} \frac{\partial a_{n}}{\partial t}-\frac{d q}{d t} f \alpha_{n}^{2} w_{n}+\tilde{U}\left(\alpha_{n} i \frac{\partial^{2} a_{n}}{\partial y^{2}}+q \frac{d^{2} f}{d y^{2}} \alpha_{n} i w_{n}\right)\right.} \\
& -\frac{d^{2} \tilde{U}}{d y^{2}}\left(\alpha_{n} i a_{n}+q f \alpha_{n} i w_{n}\right)-\tilde{U}\left(\alpha_{n}^{3} i a_{n}+q f \alpha_{n}^{3} i w_{n}\right)-\frac{1}{R e}\left[\alpha_{n}^{4} a_{n}+q f \alpha_{n}^{4} w_{n}\right. \\
& \left.\left.\left.-2\left(\alpha_{n}^{2} \frac{\partial^{2} a_{n}}{\partial y^{2}}+q \frac{d^{2} f}{d y^{2}} \alpha_{n}^{2} w_{n}\right)+\frac{\partial^{4} a_{n}}{\partial y^{4}}+q \frac{d^{4} f}{d y^{4}} w_{n}\right]\right] P_{n} \bar{P}_{k}\right\} d x=0
\end{aligned}
$$

By the orthogonality of the $P_{n}$ functions, that is

$$
\int_{0}^{L} P_{n}(x) \bar{P}_{k}(x) d x=\int_{0}^{L} e^{i n(2 \pi / L) x} e^{-i k(2 \pi / L) x} d x=\int_{0}^{L} e^{i(n-k)(2 \pi / L) x} d x=\left\{\begin{array}{l}
L, n=k \\
0, n \neq k
\end{array}\right.
$$

we get

$$
\begin{aligned}
\frac{\partial^{3} a_{k}}{\partial t \partial y^{2}} & +\frac{d q}{d t} \frac{d^{2} f}{d y^{2}} w_{k}-\alpha_{k}^{2} \frac{\partial a_{k}}{\partial t}-\frac{d q}{d t} f \alpha_{k}^{2} w_{k}+\tilde{U}\left(\alpha_{k} i \frac{\partial^{2} a_{k}}{\partial y^{2}}+q \frac{d^{2} f}{d y^{2}} \alpha_{k} i w_{k}\right) \\
& -\frac{\partial^{2} \tilde{U}}{\partial y^{2}}\left(\alpha_{k} i a_{k}+q f \alpha_{k} i w_{k}\right)-\tilde{U}\left(\alpha_{k}^{3} i a_{k}+q f \alpha_{k}^{3} i w_{k}\right)-\frac{1}{\operatorname{Re}}\left[\alpha_{k}^{4} a_{k}+q f \alpha_{k}^{4} w_{k}\right. \\
& \left.-2\left(\alpha_{k}^{2} \frac{\partial^{2} a_{k}}{\partial y^{2}}+q \frac{d^{2} f}{d y^{2}} \alpha_{k}^{2} w_{k}\right)+\frac{\partial^{4} a_{k}}{\partial y^{4}}+q \frac{d^{4} f}{d y^{4}} w_{k}\right]=\triangleq E, \text { for } k=-N, \ldots, N
\end{aligned}
$$


For the measurement equation (100), the Galerkin method yields

$$
z(t)=\sum_{k=-N}^{N} \frac{\partial^{2} a_{k}}{\partial y^{2}}(y=-1, t) P_{k}\left(x_{i}\right)+\frac{5}{2} w\left(x_{i}\right) q
$$

An interesting property of the set of equations equation (109) is that the equations are decoupled in terms of the wavenumber $\alpha_{k}$. This fact lets us study each wavenumber individually. We now rewrite equation (109) in terms of $a_{k}^{R}$ and $a_{k}^{I}$, where $a_{k}^{R}$ and $a_{k}^{I}$ are the real and imaginary parts of $a_{k}$, respectively. Since $\operatorname{real}(a)=(a+\bar{a}) / 2$ and $\operatorname{imag}(a)=(a-\bar{a}) / 2 i$, the equations for $a_{k}^{R}$ and $a_{k}^{I}$ are obtained by

$$
\frac{1}{2}(E+\bar{E})=0
$$

and

$$
\frac{1}{2 i}(E-\bar{E})=0
$$

respectively. We have that

$$
\begin{aligned}
\bar{E}= & \frac{\partial^{3} \bar{a}_{k}}{\partial t \partial y^{2}}+\frac{d q}{d t} \frac{d^{2} f}{d y^{2}} \bar{w}_{k}-\alpha^{2} \frac{\partial \bar{a}_{k}}{\partial t}-\frac{d q}{d t} f \alpha_{k}^{2} \bar{w}_{k}-\tilde{U}\left(\alpha_{k} i \frac{\partial^{2} \bar{a}_{k}}{\partial y^{2}}+q \frac{d^{2} f}{d y^{2}} \alpha_{k} i \bar{w}_{k}\right) \\
& +\frac{d^{2} \tilde{U}}{d y^{2}}\left(\alpha_{k} i \bar{a}_{k}+q f \alpha_{k} i \bar{w}_{k}\right)+\tilde{U}\left(\alpha_{k}^{3} i \bar{a}_{k}+q f \alpha_{k}^{3} i \bar{w}_{k}\right) \\
& -\frac{1}{R e}\left[\alpha_{k}^{4} \bar{a}_{k}+q f \alpha_{k}^{4} \bar{w}_{k}-2\left(\alpha_{k}^{2} \frac{\partial^{2} \bar{a}_{k}}{\partial y^{2}}+q \frac{d^{2} f}{d y^{2}} \alpha_{k}^{2} \bar{w}_{k}\right)+\frac{\partial^{4} \bar{a}_{k}}{\partial y^{4}}+q \frac{d^{4} f}{d y^{4}} \bar{w}_{k}\right]
\end{aligned}
$$

so that

$$
\begin{aligned}
\frac{1}{2}(E+\bar{E})= & \frac{\partial^{3} a_{k}^{R}}{\partial t \partial y^{2}}+\frac{d q}{d t} \frac{d^{2} f}{d y^{2}} w_{k}^{R}-\alpha_{k}^{2} \frac{\partial a_{k}^{R}}{\partial t}-\frac{d q}{d t} f \alpha_{k}^{2} w_{k}^{R}-\tilde{U}\left(\alpha_{k} \frac{\partial^{2} a_{k}^{I}}{\partial y^{2}}+q \frac{d^{2} f}{d y^{2}} \alpha_{k} w_{k}^{I}\right) \\
& +\frac{d^{2} \tilde{U}}{d y^{2}}\left(\alpha_{k} a_{k}^{I}+q f \alpha_{k} w_{k}^{I}\right)+\tilde{U}\left(\alpha_{k}^{3} a_{k}^{I}+q f \alpha_{k}^{3} w_{k}^{I}\right) \\
& -\frac{1}{R e}\left[\alpha_{k}^{4} a_{k}^{R}+q f \alpha_{k}^{4} w_{k}^{R}-2\left(\alpha_{k}^{2} \frac{\partial^{2} a_{k}^{R}}{\partial y^{2}}+q \frac{d^{2} f}{d y^{2}} \alpha_{k}^{2} w_{k}^{R}\right)+\frac{\partial^{4} a_{k}^{R}}{\partial y^{4}}+q \frac{d^{4} f}{d y^{4}} w_{k}^{R}\right]=0
\end{aligned}
$$

and

$$
\begin{aligned}
\frac{1}{2 i}(E-\bar{E})= & \frac{\partial^{3} a_{k}^{I}}{\partial t \partial y^{2}}+\frac{d q}{d t} \frac{d^{2} f}{d y^{2}} w_{k}^{I}-\alpha_{k}^{2} \frac{\partial a_{k}^{I}}{\partial t}-\frac{d q}{d t} f \alpha_{k}^{2} w_{k}^{I}+\tilde{U}\left(\alpha_{k} \frac{\partial^{2} a_{k}^{R}}{\partial y^{2}}+q \frac{d^{2} f}{d y^{2}} \alpha_{k} w_{k}^{R}\right) \\
& -\frac{d^{2} \tilde{U}}{d y^{2}}\left(\alpha_{k} a_{k}^{R}+q f \alpha_{k} w_{k}^{R}\right)-\tilde{U}\left(\alpha_{k}^{3} a_{k}^{R}+q f \alpha_{k}^{3} w_{k}^{R}\right) \\
& -\frac{1}{R e}\left[\alpha_{k}^{4} a_{k}^{I}+q f \alpha_{k}^{4} w_{k}^{I}-2\left(\alpha_{k}^{2} \frac{\partial^{2} a_{k}^{I}}{\partial y^{2}}+q \frac{d^{2} f}{d y^{2}} \alpha_{k}^{2} w_{k}^{I}\right)+\frac{\partial^{4} a_{k}^{I}}{\partial y^{4}}+q \frac{d^{4} f}{d y^{4}} w_{k}^{I}\right]=0
\end{aligned}
$$


Next, we discretize the equation in the $y$-direction. For this, we use the Chebyshev collocation method described in Section 2.7.3 on $N+1$ Chebyshev-Gauss-Labotto points as defined in equation (89). Applying the differentiation matrix $\mathscr{D}_{N}$, treating $f$ and $\tilde{U}$ and their derivatives as known functions, yields

$$
\begin{aligned}
& \mathscr{D}_{N}^{2} \frac{d \mathbf{a}_{k}^{R}}{d t}-\alpha_{k}^{2} \frac{d \mathbf{a}_{k}^{R}}{d t}=\frac{d q}{d t} \mathbf{f}_{N}^{(0)} \alpha_{k}^{2} w_{k}^{R}-\frac{d q}{d t} \mathbf{f}_{N}^{(2)} w_{k}^{R}+\tilde{U}_{N}\left(\alpha_{k} \mathscr{D}_{N}^{2} \mathbf{a}_{k}^{I}+q \mathbf{f}_{N}^{(2)} \alpha_{k} w_{k}^{I}\right) \\
& -\tilde{U}_{N}^{(2)}\left(\alpha_{k} \mathbf{a}_{k}^{I}+q \mathbf{f}_{N}^{(0)} \alpha_{k} w_{k}^{I}\right)-\tilde{U}_{N}\left(\alpha_{k}^{3} \mathbf{a}_{k}^{I}+q \mathbf{f}_{N}^{(0)} \alpha_{k}^{3} w_{k}^{I}\right) \\
& +\frac{1}{R e}\left[\alpha_{k}^{4} \mathbf{a}_{k}^{R}+q \mathbf{f}_{N}^{(0)} \alpha_{k}^{4} w_{k}^{R}-2\left(\alpha_{k}^{2} \mathscr{D}_{N}^{2} \mathbf{a}_{k}^{R}+q \mathbf{f}_{N}^{(2)} \alpha_{k}^{2} w_{k}^{R}\right)\right. \\
& \left.+\mathscr{D}_{N}^{4} \mathbf{a}_{k}^{R}+q \mathbf{f}_{N}^{(4)} w_{k}^{R}\right] \\
& \mathscr{D}_{N}^{2} \frac{d \mathbf{a}_{k}^{I}}{d t}-\alpha_{k}^{2} \frac{d \mathbf{a}_{k}^{I}}{d t}=\frac{d q}{d t} \mathbf{f}_{N}^{(0)} \alpha_{k}^{2} w_{k}^{I}-\frac{d q}{d t} \mathbf{f}_{N}^{(2)} w_{k}^{I}-\tilde{U}_{N}\left(\alpha_{k} \mathscr{D}_{N}^{2} \mathbf{a}_{k}^{R}+q \mathbf{f}_{N}^{(2)} \alpha_{k} w_{k}^{R}\right) \\
& +\tilde{U}_{N}^{(2)}\left(\alpha_{k} \mathbf{a}_{k}^{R}+q \mathbf{f}_{N}^{(0)} \alpha_{k} w_{k}^{R}\right)+\tilde{U}_{N}\left(\alpha_{k}^{3} \mathbf{a}_{k}^{R}+q \mathbf{f}_{N}^{(0)} \alpha_{k}^{3} w_{k}^{R}\right) \\
& +\frac{1}{R e}\left[\alpha_{k}^{4} \mathbf{a}_{k}^{I}+q \mathbf{f}_{N}^{(0)} \alpha_{k}^{4} w_{k}^{I}-2\left(\alpha_{k}^{2} \mathscr{D}_{N}^{2} \mathbf{a}_{k}^{I}+q \mathbf{f}_{N}^{(2)} \alpha_{k}^{2} w_{k}^{I}\right)+\mathscr{D}_{N}{ }^{4} \mathbf{a}_{k}^{I}+q \mathbf{f}_{N}^{(4)} w_{k}^{I}\right]
\end{aligned}
$$

where $\mathbf{a}_{k}^{R}$ and $\mathbf{a}_{k}^{I}$ denote the vectors

$$
\begin{aligned}
\mathbf{a}_{k}^{R} & =\left[\begin{array}{llll}
a_{k}^{R}\left(y_{0}\right) & a_{k}^{R}\left(y_{1}\right) & \ldots & a_{k}^{R}\left(y_{N}\right)
\end{array}\right]^{T} \\
\mathbf{a}_{k}^{I} & =\left[\begin{array}{llll}
a_{k}^{I}\left(y_{0}\right) & a_{k}^{I}\left(y_{1}\right) & \ldots & a_{k}^{I}\left(y_{N}\right)
\end{array}\right]^{T}
\end{aligned}
$$

and

$$
\begin{aligned}
\tilde{U}_{N}^{(m)} & =\left[\begin{array}{cccc}
\frac{d^{m} \tilde{U}}{d y^{m}}\left(y_{0}\right) & 0 & 0 & 0 \\
0 & \frac{d^{m} \tilde{U}}{d y^{m}}\left(y_{1}\right) & 0 & 0 \\
0 & 0 & \ddots & 0 \\
0 & 0 & 0 & \frac{d^{m} \tilde{U}}{d y^{m}}\left(y_{N}\right)
\end{array}\right], m=0,1,2 \ldots \\
\mathbf{f}_{N}^{(m)}= & {\left[\begin{array}{cccc}
\frac{d^{m} f}{d y^{m}}\left(y_{0}\right) & \frac{d^{m} f}{d y^{m}}\left(y_{1}\right) & \ldots & \frac{d^{m} f}{d y^{m}}\left(y_{N}\right)
\end{array}\right]^{T}, m=0,1,2, \ldots }
\end{aligned}
$$

Above, $(m)$ denotes the $m^{\text {th }}$ order derivative with respect to $y$. It remains to implement the boundary conditions. The Dirichlet boundary conditions are satisfied by simply setting $a_{k}^{R}\left(y_{0}\right)=a_{k}^{I}\left(y_{0}\right)=a_{k}^{R}\left(y_{N}\right)=a_{k}^{I}\left(y_{N}\right)=0$, and omitting the differential equations for these variables. Satisfying the Neumann boundary conditions is more involved. We start by noticing that the Neumann boundary conditions imply that

$$
\sum_{j=0}^{N} d_{0 j} a_{k}\left(y_{j}\right)=0, \quad \text { and } \sum_{j=0}^{N} d_{N j} a_{k}\left(y_{j}\right)=0
$$


where the superscripts $I$ and $R$ are omitted since the following derivation is valid for either one. The $d_{j k}$ constants in equation (116) are the elements of $\mathscr{D}_{N}$ as defined in equation (90). From equation (116), we can solve for $a_{k}\left(y_{1}\right)$ and $a_{k}\left(y_{N-1}\right)$ to obtain

$$
\begin{array}{r}
a_{k}\left(y_{1}\right)=\mathbf{l}_{1}^{T} \mathbf{a} \\
a_{k}\left(y_{N-1}\right)=\mathbf{l}_{2}^{T} \mathbf{a}
\end{array}
$$

where we have defined

$$
\left[\begin{array}{l}
\mathbf{l}_{1}^{T} \\
\mathbf{l}_{2}^{T}
\end{array}\right]=-\left[\begin{array}{ll}
d_{01} & d_{0(N-1)} \\
d_{N 1} & N(N-1)
\end{array}\right]^{-1}\left[\begin{array}{llll}
d_{02} & d_{03} & \ldots & d_{0(N-2)} \\
d_{N 2} & d_{N 3} & \ldots & d_{N(N-2)}
\end{array}\right]
$$

and

$$
\mathbf{a}=\left[\begin{array}{llll}
a_{k}\left(y_{2}\right) & a_{k}\left(y_{3}\right) & \ldots & a_{k}\left(y_{N-2}\right)
\end{array}\right]^{T}
$$

Thus, we have that

$$
\mathbf{a}_{k}=\left[\begin{array}{c}
0^{T} \\
\mathbf{l}_{1}^{T} \\
\mathscr{I}_{N-3} \\
\mathbf{l}_{2}^{T} \\
0^{T}
\end{array}\right] \mathrm{a} \triangleq \mathscr{I}_{a} \mathbf{a}
$$

Inserting equations (117) into (113) (114), and keeping in mind that the equations for $a_{k}\left(y_{0}\right), a_{k}\left(y_{1}\right), a_{k}\left(y_{N-1}\right)$, and $a_{k}\left(y_{N}\right)$ should be omitted since these variables are determined by the boundary conditions, we obtain

$$
\begin{aligned}
R\left(\mathscr{I}_{N}^{2}-\alpha_{k}^{2} \mathscr{I}\right) \mathscr{I}_{a} \dot{\mathbf{a}}^{R}= & \frac{1}{R e} R\left(\mathscr{Q}_{N}^{4} \mathscr{I}_{a}-2 \alpha_{k}^{2} \mathscr{D}_{N}^{2} \mathscr{I}_{a}+\alpha_{k}^{4} \mathscr{I}_{a}\right) \mathbf{a}^{R} \\
& +R\left(\alpha_{k} \tilde{U}_{N} \mathscr{D}_{N}^{2} \mathscr{I}_{a}-\left(\alpha_{k} \tilde{U}_{N}^{(2)}+\alpha_{k}^{3} \tilde{U}_{N}\right) \mathscr{I}_{a}\right) \mathbf{a}^{I} \\
& +w_{k}^{I} R\left(\alpha_{k} \tilde{U}_{N} \mathbf{f}_{N}^{(2)}-\left(\alpha_{k} \tilde{U}_{N}^{(2)}+\alpha_{k}^{3} \tilde{U}_{N}\right) \mathbf{f}_{N}^{(0)}\right) q \\
& +\frac{w_{k}^{R}}{R e} R\left(\alpha_{k}^{4} \mathbf{f}_{N}^{(0)}-2 \alpha_{k}^{2} \mathbf{f}_{N}^{(2)}+\mathbf{f}_{N}^{(4)}\right) q-w_{k}^{R} R\left(\mathbf{f}_{N}^{(2)}-\alpha_{k}^{2} \mathbf{f}_{N}^{(0)}\right) \dot{\mathbf{q}} \\
R\left(\mathscr{D}_{N}^{2} \quad \alpha_{k}^{2} \mathscr{I}\right) \mathscr{I}_{a} \dot{\mathbf{a}}^{I}= & -R\left(\alpha_{k} \tilde{U}_{N} \mathscr{U}_{N}^{2} \mathscr{I}_{a}-\left(\alpha_{k} \tilde{U}_{N}^{(2)}+\alpha_{k}^{3} \tilde{U}_{N}\right) \mathscr{I}_{a}\right) \mathbf{a}^{R} \\
& +\frac{1}{R e} R\left(\mathscr{\mathscr { T }}_{N}^{4} \mathscr{I}_{a}-2 \alpha_{k}^{2} \mathscr{I}_{N}^{2} \mathscr{I}_{a}+\alpha_{k}^{4} \mathscr{I}_{a}\right) \mathbf{a}^{I} \\
& -w_{k}^{R} R\left(\alpha_{k} \tilde{U}_{N} \mathbf{f}_{N}^{(2)}-\left(\alpha_{k} \tilde{U}_{N}^{(2)}+\alpha_{k}^{3} \tilde{U}_{N}\right) \mathbf{f}_{N}^{(0)}\right) q \\
& +\frac{w_{k}^{I}}{R e} R\left(\alpha_{k}^{4} \mathbf{f}_{N}^{(0)}-2 \alpha_{k}^{2} \mathbf{f}_{N}^{(2)}+\mathbf{f}_{N}^{(4)}\right) q-w_{k}^{I} R\left(\mathbf{f}_{N}^{(2)}-\alpha_{k}^{2} \mathbf{f}_{N}^{(0)}\right) \dot{\mathbf{q}}
\end{aligned}
$$

where $R$ is a matrix that selects appropriate rows, defined as

$$
R=\left[\begin{array}{lll}
\mathbf{0}_{(N-3) \times 2} & \mathscr{I}_{N-3} & \mathbf{0}_{(N-3) \times 2}
\end{array}\right.
$$


Assuming the measurement is real, we redefine $z$ as the real part of equation (110), so we get

$$
z(t)=\sum_{k=-M}^{M}\left\{\cos \left(\alpha_{k} x_{i}\right) \mathbf{s}_{1}^{T} \mathscr{D}_{N}^{2} \mathscr{I}_{a} \mathbf{a}^{R}-\sin \left(\alpha_{k} x_{i}\right) \mathbf{s}_{1}^{T} \mathscr{D}_{N}^{2} \mathscr{I}_{a} \mathbf{a}^{I}\right\}+\frac{5}{2} w\left(x_{i}\right) q
$$

where $\mathbf{s}_{j}$ is a vector of compatible dimension with a 1 at the $j^{\text {th }}$ position being the only nonzero entry. It is clear that the measurement equation (118) is not decoupled in terms of the wavenumber $\alpha_{k}$, but if we choose $w$ appropriately, we may omit all terms but one in equation (118). The reason for this will be discussed in the next section. Finally we get the system on state space form

$$
\begin{aligned}
\dot{\mathbf{x}} & =A \mathbf{x}+B u \\
z & =C \mathbf{x}+D u
\end{aligned}
$$

where

$$
\begin{aligned}
& \mathbf{x}=\left[\begin{array}{lllllll}
a_{k}^{R}\left(y_{2}\right) & \ldots & a_{k}^{R}\left(y_{N-2}\right) & a_{k}^{I}\left(y_{2}\right) & \ldots & a_{k}^{I}\left(y_{N-2}\right) & q
\end{array}\right]^{T}, \quad u=\dot{q} \\
& A=\left[\begin{array}{ccc}
M^{-1} A_{R}^{R} & M^{-1} A_{R}^{I} & M^{-1}\left(w_{k}^{I} \mathbf{q}_{1}+w_{k}^{R} \mathbf{q}_{2}\right) \\
M^{-1} A_{I}^{R} & M^{-1} A_{I}^{I} & M^{-1}\left(-w_{k}^{R} \mathbf{q}_{1}+w_{k}^{I} \mathbf{q}_{2}\right) \\
0 & 0 & 0
\end{array}\right], \quad B=\left[\begin{array}{c}
-w_{k}^{R} M^{-1} \mathbf{b} \\
-w_{k}^{I} M^{-1} \mathbf{b} \\
1
\end{array}\right] \\
& C=\left[\begin{array}{lll}
\cos \left(\alpha_{k} x_{i}\right) \mathbf{s}_{N}^{T} \mathscr{D}_{N}^{2} \mathscr{I}_{a} & -\sin \left(\alpha_{k} x_{i}\right) \mathbf{s}_{N}^{T} \mathscr{D}_{N}^{2} \mathscr{I}_{a} & \frac{5}{2} w\left(x_{i}\right)
\end{array}\right], \quad D=0 \\
& M=R\left(\mathscr{D}_{N}^{2}-\alpha_{k}^{2} \mathscr{I}\right) \mathscr{I}_{a} \\
& A_{R}^{R}=A_{I}^{I}=\frac{1}{R e} R\left(\mathscr{D}_{N}^{4} \mathscr{I}_{a}-2 \alpha_{k}^{2} \mathscr{D}_{N}^{2} \mathscr{I}_{a}+\alpha_{k}^{4} \mathscr{I}_{a}\right) \\
& A_{R}^{I}=-A_{I}^{R}=R\left(\alpha_{k} \tilde{U}_{N} \mathscr{D}_{N}^{2} \mathscr{I}_{a}-\left(\alpha_{k} \tilde{U}_{N}^{(2)}+\alpha_{k}^{3} \tilde{U}_{N}\right) \mathscr{I}_{a}\right) \\
& \mathbf{q}_{1}=R\left(\alpha_{k} \tilde{U}_{N} \mathbf{f}_{N}^{(2)}-\left(\alpha_{k} \tilde{U}_{N}^{(2)}+\alpha_{k}^{3} \tilde{U}_{N}\right) \mathbf{f}_{N}^{(0)}\right), \mathbf{q}_{2}=\frac{1}{R e} R\left(\alpha_{k}^{4} \mathbf{f}_{N}^{(0)}-2 \alpha_{k}^{2} \mathbf{f}_{N}^{(2)}+\mathbf{f}_{N}^{(4)}\right) \\
& \mathbf{b}=R\left(\mathbf{f}_{N}^{(2)}-\alpha_{k}^{2} \mathbf{f}_{N}^{(0)}\right), R=\left[\begin{array}{lll}
\mathbf{0}_{(N-3) \times 2} & \mathscr{I}_{N-3} & \mathbf{0}_{(N-3) \times 2}
\end{array}\right]
\end{aligned}
$$

4.1.2. Classical control In the previous section we developed a state space representation of the discretized 2D linearized channel flow equations. Apart from the measurement equation, the resulting system is decoupled in terms of the wave number. It turns out that the system has one complex conjugate pair of eigenvalues in the right half of the complex plane for $k=2$. All other poles of the system are in the left half of the complex plane (except for the pole at the origin, due to the boundary control input configuration). Figure 7 shows poles and zeros for $k=2$ at Reynolds number 10000 and $N=150$ (i.e. 151 collocation points), and with the shear measurement taken at $\left.x_{i}=\pi, w(x)=\sin (2 \pi k / L) x\right)$, and $L=4 \pi$. With this choice of $w(x),(A, B)$ is rendered uncontrollable for all wave numbers with $k \neq 2$. Therefore, the fact that the 


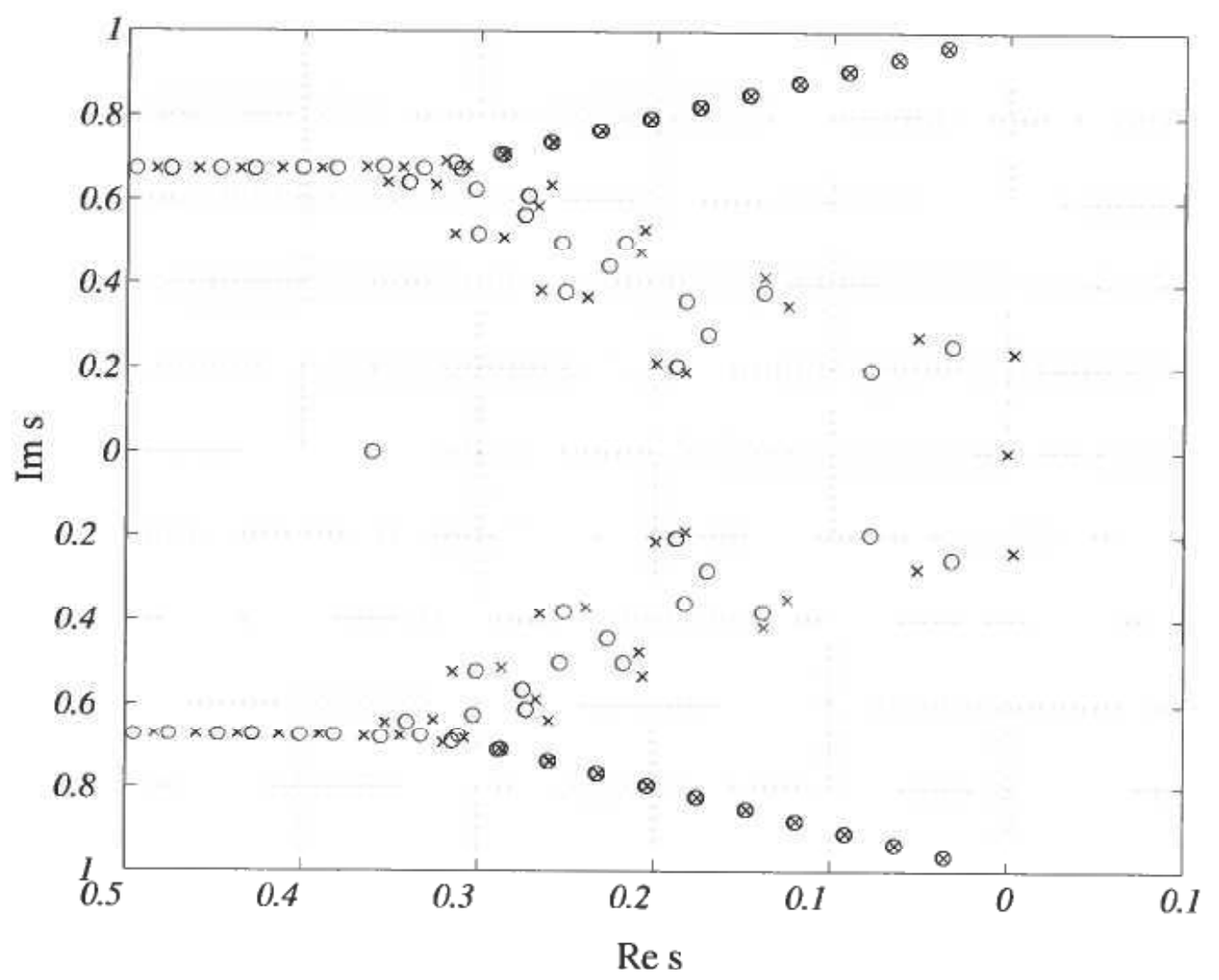

Figure 7. Poles $(x)$ and zeros $(O)$ for the system.

measurement does not decouple in terms of the wavenumber does not present a problem, since the control will be unable to destabilize any pole of sub-systems with $k \neq 2$. Figure 8 shows the Nyquist plot. The Nyquist stability criterion indicates that when $-1 / K \in(-105,0)$ (approximately), no poles of the closed loop system lie in the right half plane, which corresponds to $K>0.0095$. This result is confirmed by Figure 9 , which shows the real component of the system's least stable eigenvalue as a function of feedback gain $K$.

4.1.3. $L Q G$ control Assuming that equations (119)-(120) are subjected to additive disturbances, $w_{d}$ and $w_{n}$, that are uncorrelated Gaussian stochastic processes with zero means and covariances $W$, and $V$, respectively, it is straight forward to apply LQG control theory. For illustration, we select $W=Q=I$ and $V=R=1$, and construct a standard LQG controller. Here $Q$ is the penalty on the states, and $R$ is the penalty on the control input. Figure 10 shows the result in terms of the system poles for the open-loop system, the state feedback closed-loop system, and the output feedback closed-loop system. Clearly, the open-loop unstable poles are moved into the left half of the complex plane by the control.

4.1.4. Implementation Although the state space model (119)-(120) takes the form of a single-input-single-output (SISO) system, with input $u=\dot{q}(t)$ and output $z=\partial u$ I $\partial y\left(x_{i},-1, t\right)$, the realization of the control system is distributed. This follows from the fact that the actuation is blowing and suction of fluid distributed along the wall as a cosine in $x(d w / d x$, where $w$ is a sine in $x)$, whose amplitude is altered with a 


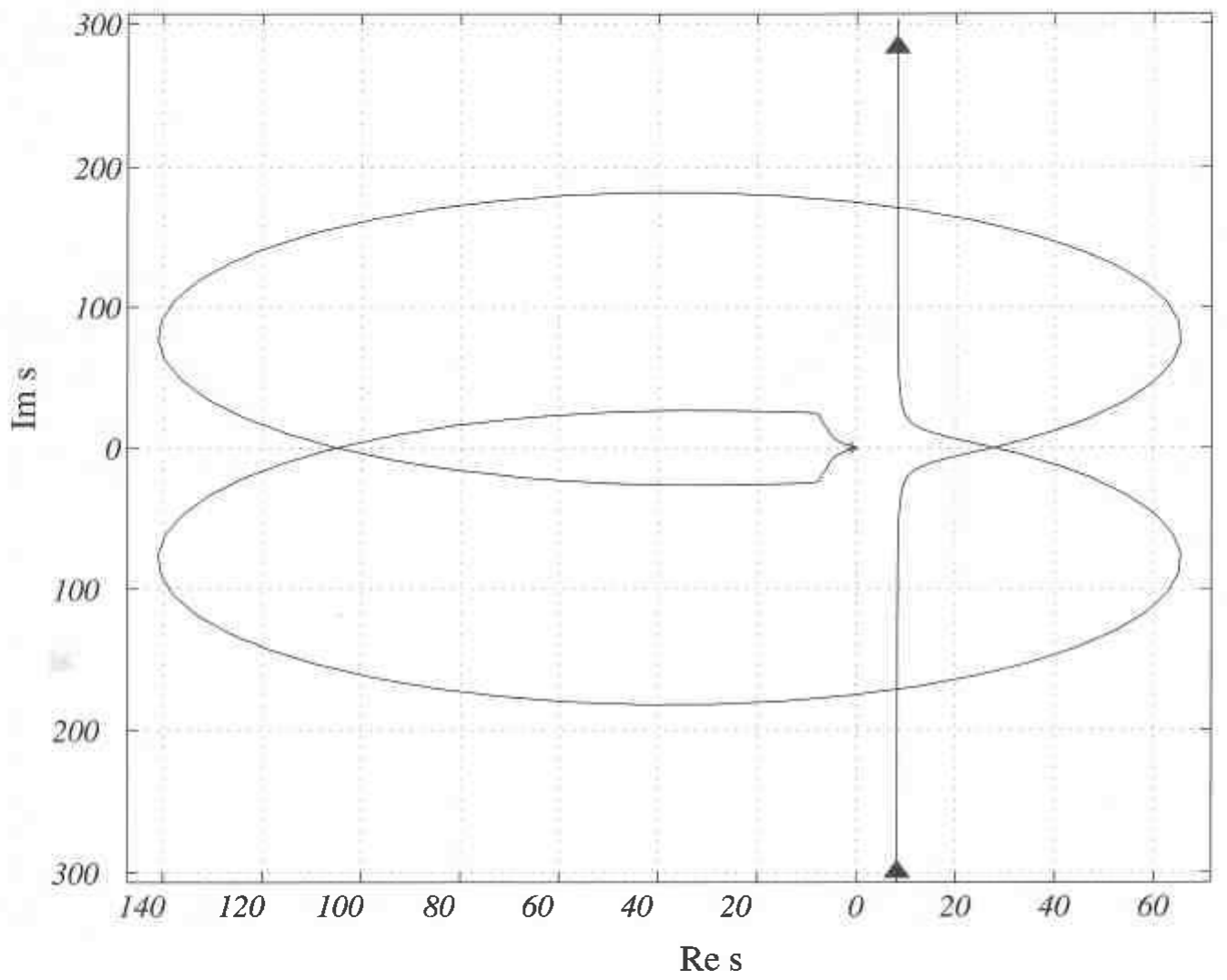

Figure 8. Nyquist plot.

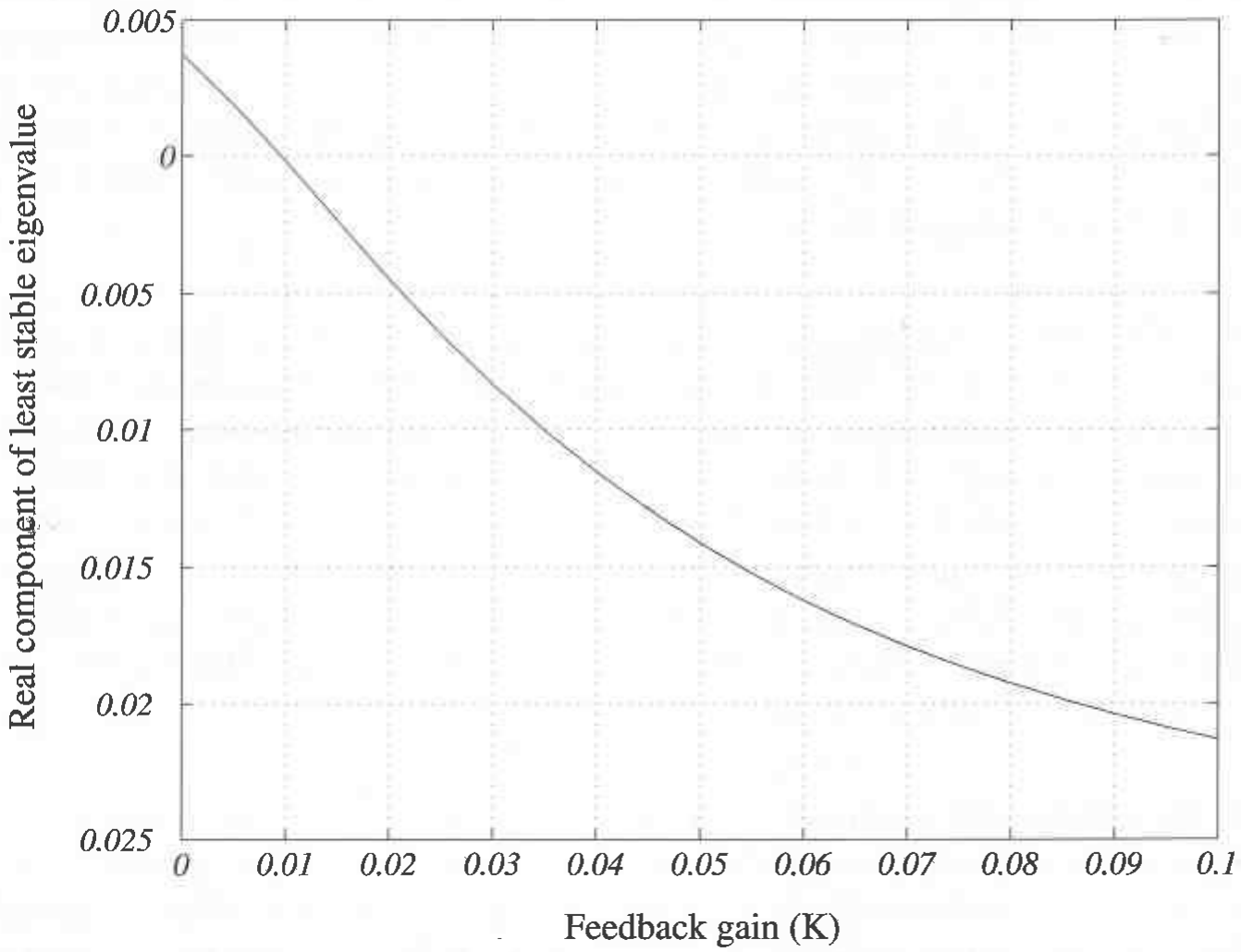

Figure 9. Real component of the least stable eigenvalue as a function of feedback gain $K$. 

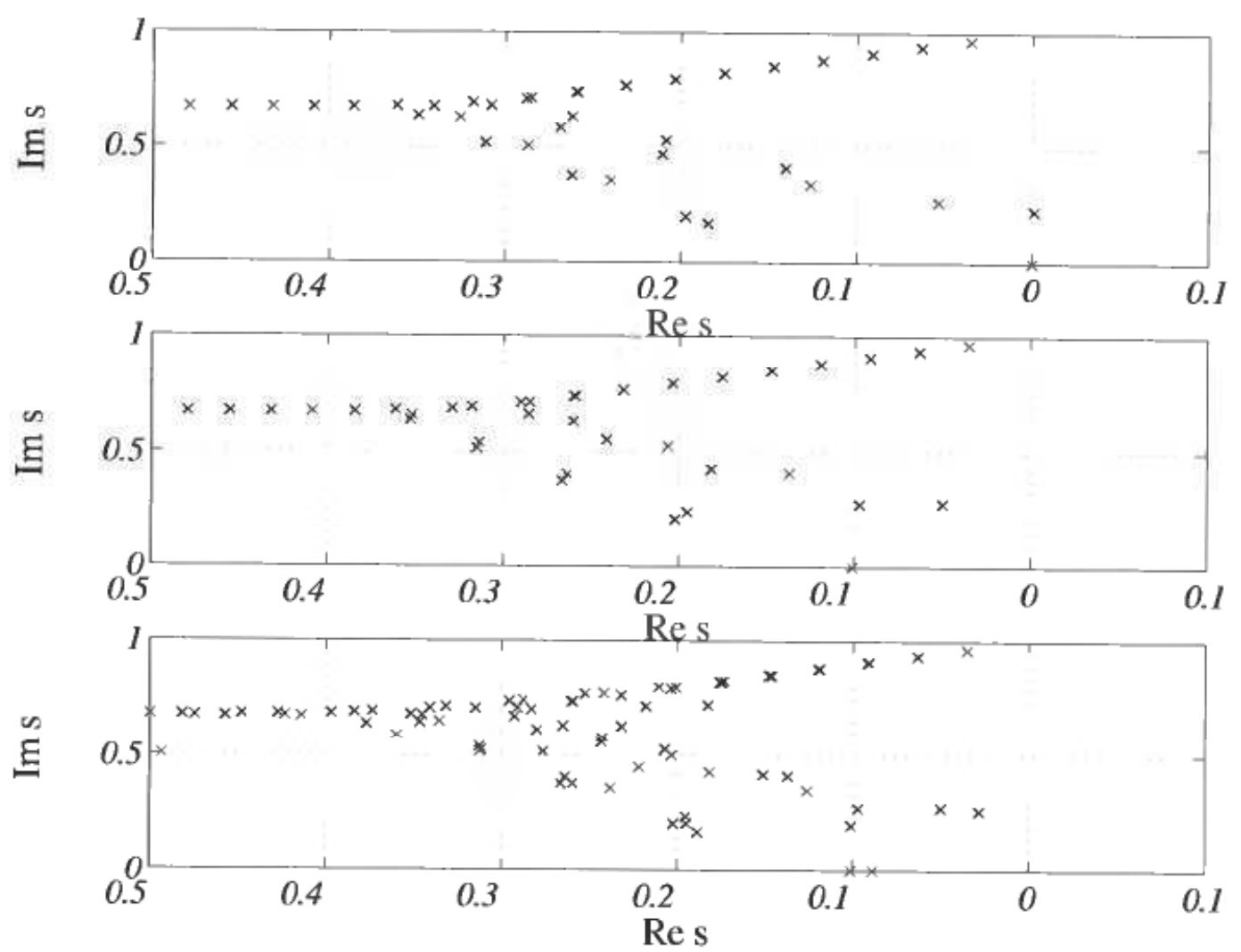

Figure 10. LQG synthesis: system poles for the open-loop system (top), the state feedback closed-loop system (middle), \%and the output feedback closed-loop system (bottom).

speed given by the control input $u=\dot{q}(t)$. The control law is centralized since the control signal must reach all actuators on the wall. Centralized versus decentralized control schemes are discussed in more detail in Section 5.

\subsection{D channel flow}

4.2.1. Reduced order model In Bewley and Liu (1998) linear control is applied to the $3 \mathrm{D}$ channel flow. In this case, shear measurements in two directions ( $\partial U / \partial y$ and $\partial W /$ $\partial y$ ) are taken at every point on both walls, and actuation is applied in the form of wall transpiration on both walls. The point of departure is the linearized NavierStokes equations for 3D channel flow (55)-(58), which may be rewritten in terms of the wall-normal velocity component $v$, and the wall-normal vorticity component, denoted $\omega$ (vorticity is defined as $\partial \omega=\operatorname{curl}(\mathbf{W})$ ). Taking the Laplacian of equation (57) yields

$$
\begin{aligned}
& \frac{\partial}{\partial t}\left(\frac{\partial^{2} v}{\partial x^{2}}+\frac{\partial^{2} v}{\partial y^{2}}+\frac{\partial^{2} v}{\partial z^{2}}\right)+\tilde{U} \frac{\partial}{\partial x}\left(\frac{\partial^{2} v}{\partial x^{2}}+\frac{\partial^{2} v}{\partial y^{2}}+\frac{\partial^{2} v}{\partial z^{2}}\right)+\frac{\partial^{2} \tilde{U}}{\partial y^{2}} \frac{\partial v}{\partial x}+2 \frac{\partial \tilde{U}}{\partial y} \frac{\partial^{2} v}{\partial x \partial y} \\
= & -\frac{\partial}{\partial y}\left(\frac{\partial^{2} p}{\partial x^{2}}+\frac{\partial^{2} p}{\partial y^{2}}+\frac{\partial^{2} p}{\partial z^{2}}\right) \\
& +\frac{1}{R e}\left(\frac{\partial^{4} v}{\partial x^{4}}+\frac{\partial^{4} v}{\partial y^{4}}+\frac{\partial^{4} v}{\partial z^{4}}+2 \frac{\partial^{4} v}{\partial x^{2}} \partial y^{2}+2 \frac{\partial^{4} v}{\partial x^{2}} \partial z^{2}+2 \frac{\partial^{4} v}{\partial y^{2}} \partial z^{2}\right)
\end{aligned}
$$


Taking the divergence of equations (56)-(58) yields

$$
\begin{aligned}
& \frac{\partial}{\partial \mathrm{t}}\left(\frac{\partial u}{\partial x}+\frac{\partial v}{\partial y}+\frac{\partial w}{\partial z}\right)+\tilde{U} \frac{\partial}{\partial x}\left(\frac{\partial u}{\partial x}+\frac{\partial v}{\partial y}+\frac{\partial w}{\partial z}\right)+2 \frac{d \tilde{U}}{d y} \frac{\partial v}{\partial x}=-\frac{\partial^{2} p}{\partial x^{2}}-\frac{\partial^{2} p}{\partial y^{2}}-\frac{\partial^{2} p}{\partial z^{2}} \\
& +\frac{1}{R e}\left(\frac{\partial^{2}}{\partial x^{2}}\left(\frac{\partial u}{\partial x}+\frac{\partial v}{\partial y}+\frac{\partial w}{\partial z}\right)+\frac{\partial^{2}}{\partial y^{2}}\left(\frac{\partial u}{\partial x}+\frac{\partial v}{\partial y}+\frac{\partial w}{\partial z}\right)+\frac{\partial^{2}}{\partial z^{2}}\left(\frac{\partial u}{\partial x}+\frac{\partial v}{\partial y}+\frac{\partial w}{\partial z}\right)\right)
\end{aligned}
$$

and using continuity equation (55), we obtain

$$
2 \frac{d \tilde{U}}{d y} \frac{\partial v}{\partial x}=-\frac{\partial^{2} p}{\partial x^{2}}-\frac{\partial^{2} p}{\partial y^{2}}-\frac{\partial^{2} p}{\partial z^{2}}
$$

Inserting equation (122) into (121) yields the equation for the wall-normal velocity component, $v$, as

$$
\begin{aligned}
\frac{\partial}{\partial t}\left(\frac{\partial^{2} v}{\partial x^{2}}+\frac{\partial^{2} v}{\partial y^{2}}+\frac{\partial^{2} v}{\partial z^{2}}\right)= & -\tilde{U} \frac{\partial}{\partial x}\left(\frac{\partial^{2} v}{\partial x^{2}}+\frac{\partial^{2} v}{\partial y^{2}}+\frac{\partial^{2} v}{\partial z^{2}}\right)+\frac{d^{2} \tilde{U}}{d y^{2}} \frac{\partial v}{\partial x} \\
& +\frac{1}{R e}\left(\frac{\partial^{4} v}{\partial x^{4}}+\frac{\partial^{4} v}{\partial y^{4}}+\frac{\partial^{4} v}{\partial z^{4}}+2 \frac{\partial^{4} v}{\partial x^{2}} \partial y^{2}+2 \frac{\partial^{4} v}{\partial x^{2}} \partial z^{2}+2 \frac{\partial^{4} v}{\partial y^{2}} \partial z^{2}\right)
\end{aligned}
$$

The wall-normal component of vorticity is $\omega=\partial u / \partial z-\partial w / \partial x$. Thus, taking the partial derivative of equation (56) with respect to $z$ and subtracting the partial derivative of equation (58) with respect to $x$, yields

$$
\frac{\partial \omega}{\partial t}=-\frac{d \tilde{U}}{d y} \frac{\partial v}{\partial z}-\tilde{U} \frac{\partial \omega}{\partial x}+\frac{1}{R e}\left(\frac{\partial^{2} \omega}{\partial x^{2}}+\frac{\partial^{2} \omega}{\partial y^{2}}+\frac{\partial^{2} \omega}{\partial z^{2}}\right)
$$

The control is applied as an unsteady boundary condition on the wall-normal velocity component $v$, and the no-slip condition in the $x$ and $z$ directions leads to homogeneous Dirichlet boundary conditions for $\omega$, that is

$$
\omega(x, y= \pm 1, z, t)=0
$$

By continuity, $\partial v / \partial y=0$ at the wall, so equation (123) is also subject to homogeneous Neumann boundary conditions. Expanding $v$ and $\omega$ as

$$
\begin{aligned}
& v(x, y, z, t)=\sum_{k_{x}, k_{z}} \hat{v}\left(k_{x}, y, k_{z}, t\right) e^{i\left(\left(2 \pi k_{x} / L_{x}\right) x+\left(2 \pi k_{z} / L_{z}\right) z\right)} \\
& \omega(x, y, z, t)=\sum_{k_{x}, k_{z}} \hat{\omega}\left(k_{x}, y, k_{z}, t\right) e^{i\left(\left(2 \pi k_{x} / L_{x}\right) x+\left(2 \pi k_{z} / L_{z}\right) z\right)}
\end{aligned}
$$

the Fourier-Galerkin method yields

$$
\begin{aligned}
\frac{\partial}{\partial t}\left(-\alpha_{x}^{2} \hat{v}+\frac{\partial^{2} \hat{v}}{\partial y^{2}}-\alpha_{z}^{2} \hat{v}\right)= & \tilde{U}\left(i \alpha_{x}^{3} \hat{v}-i \alpha_{x} \frac{\partial^{2} \hat{v}}{\partial y^{2}}+i \alpha_{x} \alpha_{z}^{2} \hat{v}\right)+\frac{\partial^{2} \tilde{U}}{\partial y^{2}} i \alpha_{x} \hat{v} \\
& +\frac{1}{R e}\left(\alpha_{x}^{4} \hat{v}+\frac{\partial^{4} \hat{v}}{\partial y^{4}}+\alpha_{z}^{4} \hat{v}-2 \alpha_{x}^{2} \frac{\partial^{2} \hat{v}}{\partial y^{2}}+2 \alpha_{x}^{2} \alpha_{z}^{2} \hat{v}-2 \alpha_{z}^{2} \frac{\partial^{2} \hat{v}}{\partial y^{2}}\right) \\
\frac{\partial \hat{\omega}}{\partial t}= & -\frac{\partial \tilde{U}}{\partial y} i \alpha_{z} \hat{v}-\tilde{U} i \alpha_{x} \hat{\omega}+\frac{1}{\operatorname{Re}}\left(-\alpha_{x}^{2} \hat{\omega}+\frac{\partial^{2} \hat{\omega}}{\partial y^{2}}-\alpha_{z}^{2} \hat{\omega}\right)
\end{aligned}
$$


where

$$
\begin{aligned}
& \alpha_{x}=\frac{2 \pi k_{x}}{L_{x}} \\
& \alpha_{x}=\frac{2 \pi k_{z}}{L_{x}}
\end{aligned}
$$

As in Section 4.1 we discretize the wall-normal direction on $N+1$ Chebyshev-GaussLobatto equation (89) points using the Chebyshev-collocation method. Applying the differentiation matrix $\mathscr{T}_{N}$, treating $\tilde{U}$ and its derivatives as known functions, yields

$$
\begin{aligned}
& \left(\mathscr{D}_{N}^{2}-\left(\alpha_{x}^{2}+\alpha_{z}^{2}\right) \mathscr{T}\right) \frac{d \hat{\mathbf{v}}\}_{a}}{d t}=\left(\tilde{U}_{N}^{(0)}\left(-i \alpha_{x} \mathscr{N}_{N}^{2}+\left(i \alpha_{x}^{3}+i \alpha_{x} \alpha_{z}^{2}\right) \mathscr{I}\right)+\tilde{U}_{N}^{(2)} i \alpha_{x}\right. \\
& \left.+\frac{1}{\operatorname{Re}}\left(\mathscr{Q}_{N}^{4}-2\left(\alpha_{x}^{2}+\alpha_{z}^{2}\right) \mathscr{Q}_{N}^{2}+\left(\alpha_{x}^{2}+\alpha_{z}^{2}\right)^{2} \mathscr{I}\right)\right) \hat{\mathbf{v}}_{a} \\
& \frac{d \hat{\omega}_{a}}{d t}=-\tilde{U}_{N}^{(1)} i \alpha_{z} \hat{\mathbf{v}}_{a}+\left(-\tilde{U}_{N}^{(0)} i \alpha_{x}+\frac{1}{R e}\left(\mathscr{D}_{N}^{2}-\left(\alpha_{x}^{2}+\alpha_{z}^{2}\right) \mathscr{I}\right)\right) \partial \hat{\omega}_{a}
\end{aligned}
$$

where

$$
\begin{aligned}
\hat{\mathbf{v}}_{a} & =\left[\begin{array}{llll}
\hat{v}\left(y_{0}\right) & \hat{v}\left(y_{1}\right) & \ldots & \hat{v}\left(y_{N}\right)
\end{array}\right]^{T} \\
\hat{\omega}_{a} & =\left[\begin{array}{llll}
\hat{\omega}\left(y_{0}\right) & \hat{\omega}\left(y_{1}\right) & \ldots & \hat{\omega}\left(y_{N}\right)
\end{array}\right]^{T}
\end{aligned}
$$

and $\tilde{U}_{N}^{(m)}$ is defined as in equation (115). Since the time derivative of $\hat{v}\left(y_{0}\right)$ and $\hat{v}\left(y_{N}\right)$ occur in equations (125)-(126), we let the time derivative of $\hat{v}\left(y_{0}\right)$ and $\hat{v}\left(y_{N}\right)$ be the control input. The Neumann boundary conditions on $v$ imply that

$$
\sum_{j=0}^{N} d_{0 j} \hat{v}\left(y_{j}\right)=0, \text { and } \sum_{j=0}^{N} d_{N j} \hat{v}\left(y_{j}\right)=0
$$

Solving equation (127) for $\hat{v}\left(y_{1}\right)$ and $\hat{v}\left(y_{N-1}\right)$, yields

$$
\begin{array}{r}
\hat{v}\left(y_{1}\right)=\mathbf{l}_{1}^{T} \hat{\mathbf{v}} \\
\hat{v}\left(y_{N-1}\right)=\mathbf{l}_{2}^{T} \hat{\mathbf{v}}
\end{array}
$$

where we have defined

$$
\begin{aligned}
& {\left[\begin{array}{l}
\mathbf{l}_{1}^{T} \\
\mathbf{l}_{2}^{T}
\end{array}\right]=-\left[\begin{array}{ll}
d_{01} & d_{0(N-1)} \\
d_{N 1} & d_{N(N-1)}
\end{array}\right]^{-1}\left[\begin{array}{lllll}
d_{00} & d_{02} & \ldots & d_{0(N-2)} & d_{0 N} \\
d_{N 0} & d_{N 2} & \ldots & d_{N(N-2)} & d_{N N}
\end{array}\right] \text {, and }} \\
& \hat{\mathbf{v}}=\left[\begin{array}{lllll}
\hat{v}\left(y_{0}\right) & \hat{v}\left(y_{2}\right) & \ldots & \hat{v}\left(y_{N-2}\right) & \hat{v}\left(y_{N}\right)
\end{array}\right.
\end{aligned}
$$

Thus, we get

$$
\hat{\mathbf{v}}_{a}=\left[\begin{array}{c}
\mathbf{s}_{1}^{T} \\
\mathbf{l}_{1}^{T} \\
\mathscr{I}_{N-3} \\
\mathbf{l}_{2}^{T} \\
\mathbf{s}_{N-3}^{T}
\end{array}\right] \hat{\mathbf{v}} \triangleq \mathscr{I}_{v} \hat{\mathbf{v}}
$$


and, defining $\left.\hat{\omega} \triangleq \hat{\omega}\left(y_{1}\right) \quad \hat{\omega}\left(y_{2}\right) \quad \ldots \quad \hat{\omega}\left(y_{N-1}\right)\right]^{T}$, the homogeneous Dirichlet boundary conditions on $\omega$ yields

$$
\hat{\omega}_{a}=\left[\begin{array}{c}
\boldsymbol{0}^{T} \\
\mathscr{I}_{N-1} \\
\boldsymbol{0}^{T}
\end{array}\right] \hat{\omega} \triangleq \mathscr{I}_{o} \hat{\omega}
$$

Inserting equations (130) and (131) into (125)-(126), omitting the equations for $\hat{v}\left(y_{1}\right)$, and $\hat{v}\left(y_{N-1}\right)$ (since they are determined by the homogeneous Neumann boundary conditions), and omitting the equations for $\hat{\omega}\left(y_{0}\right)$ and $\hat{\omega}\left(y_{N}\right)$ (since they are zero due to the homogeneous Dirichlet boundary conditions), we get

$$
\begin{gathered}
\frac{d \hat{\mathbf{v}}}{d t}=M_{1}^{-1} R_{1}\left(\tilde{U}_{N}^{(0)}\left(-i \alpha_{x} \mathscr{D}_{N}^{2}+i \alpha_{x} \alpha \mathscr{I}\right)+\tilde{U}_{N}^{(2)} i \alpha_{x}+\frac{1}{R e}\left(\mathscr{D}_{N}^{4}-2 \alpha \mathscr{D}_{N}^{2}+\alpha^{2} \mathscr{I}\right)\right) \mathscr{I}_{v} \hat{\mathbf{v}} \\
\frac{d \hat{\omega}}{d t}=-M_{2}^{-1} R_{2} \tilde{U}_{N}^{(1)} i \alpha_{z} \mathscr{I}_{v} \hat{\mathbf{v}}+M_{2}^{-1} R_{2}\left(-\tilde{U}_{N}^{(0)} i \alpha_{x}+\frac{1}{R e}\left(\mathscr{D}_{N}^{2}-\alpha \mathscr{I}\right)\right) \mathscr{I}_{\omega} \hat{\omega}
\end{gathered}
$$

where $\alpha=\alpha_{x}^{2}+\alpha_{z}^{2}$, and

$$
\begin{aligned}
M_{1} & =R_{1}\left(\mathscr{D}_{N}^{2}-\left(\alpha_{x}^{2}+\alpha_{z}^{2}\right) \mathscr{T}\right) \mathscr{I}_{v} \\
M_{2} & =R_{2} \mathscr{I}_{\omega} \\
R_{1} & =\left[\begin{array}{cccccc}
1 & 0 & \ldots & 0 & 0 \\
0 & 0 & \mathscr{I}_{N-3} & 0 & 0 \\
0 & 0 & \ldots & 0 & 1
\end{array}\right] \\
R_{2} & =\left[\begin{array}{llll}
0 & \mathscr{I}_{N-1} & 0
\end{array}\right]
\end{aligned}
$$

The measurement $\hat{z}$ is the following

$$
\hat{z}\left(\alpha_{x}, \alpha_{z}, t\right)=\frac{1}{R e}\left[\begin{array}{c}
-\frac{\partial \hat{u}}{\partial y}\left(\alpha_{x}, y=1, \alpha_{z}, t\right) \\
\frac{\partial \hat{u}}{\partial y}\left(\alpha_{x}, y=-1, \alpha_{z}, t\right) \\
-\frac{\partial \hat{w}}{\partial y}\left(\alpha_{x}, y=1, \alpha_{z}, t\right) \\
\frac{\partial \hat{w}}{\partial y}\left(\alpha_{x}, y=-1, \alpha_{z}, t\right)
\end{array}\right]
$$

which is obtained physically by measuring the shear in two directions ( $\partial U / \partial y$ and $\partial W / \partial y)$ on the entire wall. From continuity equation (55) we have that

$$
i \alpha_{x} \hat{u}+\frac{\partial \hat{v}}{\partial y}+i \alpha_{z} \hat{w}=0
$$

which, along with $\hat{\omega}=i \alpha_{z} \hat{u}-i \alpha_{x} \hat{w}$, yield 


$$
\begin{aligned}
& \hat{u}=\frac{i}{\alpha}\left(\alpha_{x} \frac{\partial \hat{v}}{\partial y}-\alpha_{z} \hat{\omega}\right) \\
& \hat{w}=\frac{i}{\alpha}\left(\alpha_{z} \frac{\partial \hat{v}}{\partial y}+\alpha_{x} \hat{\omega}\right)
\end{aligned}
$$

So the measurement is

$$
\hat{z}\left(\alpha_{x}, \alpha_{z}, t\right)=\frac{i}{\alpha R e}\left[\begin{array}{c}
-\alpha_{x} \mathbf{s}_{N+1}^{T} \mathscr{D}_{N}^{2} \mathscr{I}_{v} \hat{\mathbf{v}}+\alpha_{z} \mathbf{s}_{N+1}^{T} \mathscr{D}_{N}^{1} \mathscr{I}_{\omega} \hat{\omega} \\
\alpha_{x} \mathbf{s}_{1}^{T} \mathscr{D}_{N}^{(2)} \mathscr{I}_{v} \hat{\mathbf{v}}-\alpha_{z} \mathbf{s}_{1}^{T} \mathscr{\mathscr { I }}_{N}^{1} \mathscr{I}_{\omega} \hat{\omega} \\
-\alpha_{z} \mathbf{s}_{N+1}^{T} \mathscr{V}_{N}^{(2)} \mathscr{I}_{v} \hat{\mathbf{v}}-\alpha_{x} \mathbf{s}_{N+1}^{T} \mathscr{D}_{N}^{1} \mathscr{I}_{\omega} \hat{\omega} \\
\alpha_{z} \mathbf{s}_{1}^{T} \mathscr{D}_{N}^{(2)} \mathscr{I}_{v} \hat{\mathbf{v}}+\alpha_{x} \mathbf{s}_{1}^{T} \mathscr{Q}_{N}^{1} \mathscr{I}_{\omega} \hat{\omega}
\end{array}\right]
$$

Finally, replacing the equations for $\hat{v}\left(y_{0}\right)$ and $\hat{v}\left(y_{N}\right)$ in equation (132) by the control input. we get the system on state space form

$$
\begin{aligned}
\dot{\mathbf{x}} & =A \mathbf{x}+B \mathbf{u} \\
z & =C \mathbf{x}+D \mathbf{u}
\end{aligned}
$$

where

$$
\begin{aligned}
& \mathbf{x}=\left[\begin{array}{ll}
\hat{\mathbf{v}}^{T} & \hat{\omega}^{T}
\end{array}\right]^{T}, \quad \mathbf{u}=\left[\begin{array}{ll}
\dot{\hat{v}}\left(y_{0}\right) & \dot{\hat{v}}\left(y_{N}\right)
\end{array}\right]^{T} \\
& A=\left[\begin{array}{cc}
A_{11} & 0 \\
A_{21} & A_{22}
\end{array}\right], \quad B=\left[\begin{array}{c}
\mathscr{I}_{b} \\
0
\end{array}\right] \\
& C=\frac{i}{\operatorname{Re}\left(\alpha_{z}^{2}+\alpha_{x}^{2}\right)}\left[\begin{array}{cc}
-\alpha_{x} \mathbf{s}_{N+1}^{T} \mathscr{D}_{N}^{2} \mathscr{I}_{v} & \alpha_{z} \mathbf{s}_{N+1}^{T} \mathscr{I}_{N}^{1} \mathscr{I}_{\omega} \\
\alpha_{x} \mathbf{s}_{1}^{T} \mathscr{D}_{N}^{(2)} \mathscr{I}_{v} & -\alpha_{z} \mathbf{s}_{1}^{T} \mathscr{D}_{N}^{1} \mathscr{I}_{\omega} \\
-\alpha_{z} \mathbf{s}_{N+1}^{T} \mathscr{D}_{N}^{(2)} \mathscr{I}_{v} & -\alpha_{x} \mathbf{s}_{N+1}^{T} \mathscr{D}_{N}^{1} \mathscr{I}_{\omega} \\
\chi_{z} \mathbf{s}_{1}^{T} \mathscr{D}_{N}^{(2)} \mathscr{I}_{v} & \alpha_{x} \mathbf{s}_{1}^{T} \mathscr{I}_{N}^{1} \mathscr{I}_{\omega}
\end{array}\right], \quad D=\mathbf{0}_{4 \times 2} \\
& A_{11}=R_{3} M_{1}^{-1} R_{1}\left(\tilde{U}_{N}^{(0)}\left(-i \alpha_{x} \mathscr{D}_{N}^{2}+\left(i \alpha_{x}^{3}+i \alpha_{x} \alpha_{z}^{2}\right) \mathscr{I}\right)+\tilde{U}_{N}^{(2)} i \alpha_{x}\right. \\
& \left.+\frac{1}{R e}\left(\mathscr{D}_{N}^{4}-2\left(\alpha_{x}^{2}+\alpha_{z}^{2}\right) \mathscr{L}_{N}^{2}+\left(\alpha_{x}^{2}+\alpha_{z}^{2}\right)^{2} \mathscr{I}\right)\right) \mathscr{I}_{v} \\
& A_{21}=-M_{2}^{-1} R_{2} \tilde{U}_{N}^{(1)} i \alpha_{z} \mathscr{I}_{v}, A_{22}=M_{2}^{-1} R_{2}\left(-\tilde{U}_{N}^{(0)} i \alpha_{x}+\frac{1}{R e}\left(\mathscr{P}_{N}^{2}-\left(\alpha_{x}^{2}+\alpha_{z}^{2}\right) \mathscr{I}\right)\right) \mathscr{I}_{\omega} \\
& \mathscr{I}_{b}=\left[\begin{array}{ccccc}
1 & 0 & \ldots & 0 & 0 \\
0 & 0 & \ldots & 0 & 1
\end{array}\right]_{2 \times(N-1)}^{T} \\
& R_{3}=\left[\begin{array}{ccc}
0 & \cdots & 0 \\
\vdots & \mathscr{I}_{N-3} & \vdots \\
0 & \cdots & 0
\end{array}\right]
\end{aligned}
$$


Note that the rigorous derivation of the homogeneous Neumann boundary conditions on $v$ removes the spurious eigenvalues reported in Bewley and Liu (1998), and thus removes the need for redesigning the matrix $A$ to damp out these modes, which is done in that reference.

4.2.2. Control strategies: A comparative study In Bewley and Liu (1998), two cases are studied in detail: (1) Reynolds number $10000, \alpha_{x}=2$, and $\alpha_{z}=0$; and (2) Reynolds number $5000, \alpha_{x}=0$, and $\alpha_{z}=2.044$. In case (1), where $\alpha_{z}=0$, the equations for $\omega$ decouples from the equation for $v$ as well as from the control input, and the problem becomes the same as that studied in the previous sections. Case (2) is a different problem in that it is subcritical, which means that there are no unstable modes. The control problem is nevertheless interesting because perturbations in laminar subcritical flows may lead to transition to turbulence. Therefore, it is of interest to apply control in order to delay, or maybe even prevent, transition to turbulence. The particular case chosen here is the pair $\left(\alpha_{x}, \alpha_{z}\right)$ that gives the maximum transient energy growth, as shown in Butler and Farrell (1992). The 'worst-case' transient energy growth is defined as

$$
\mathscr{E}(t)=\sup _{x_{0}} \frac{\|x(t)\|_{2}}{\left\|x_{0}\right\|_{2}}
$$

The state space model developed in the previous section is subjected to state disturbances, $w_{1}$, and measurement noise, $w_{2}$, such that it can be written

$$
\begin{aligned}
& \dot{x}=A x+B_{1} w+B_{2} u \\
& y=C_{2} x+D_{21} w
\end{aligned}
$$

where

$$
w=\left[\begin{array}{l}
w_{1} \\
w_{2}
\end{array}\right], \quad B_{1}=\left[\begin{array}{ll}
I & 0
\end{array}\right], \quad B_{2}=B, \quad C_{2}=C, \quad \text { and } \quad D_{21}=\left[\begin{array}{ll}
0 & \alpha I
\end{array}\right]
$$

The performance variable, $z$, is defined as

$$
z=C_{1} x+D_{12} u
$$

where

$$
C_{1}=\left[\begin{array}{c}
Q^{1 / 2} \\
0
\end{array}\right], \quad D_{12}=\left[\begin{array}{l}
0 \\
l I
\end{array}\right]
$$

The system is now on the standard form needed for application of the $\mathscr{H}_{2}$ and $\mathscr{H}_{\infty}$ control strategies. $Q$, which shapes the dependence of the performance upon the states, is chosen such that $x^{*} Q x$ is related to the energy of the flow perturbations, which appears to be the best choice for delaying transition to turbulence (Bewley, 2001). In Bewley and Liu (1998), an extensive parametric study is carried out, quantifying the performance of $\mathscr{H}_{2}, \mathscr{H}_{\infty}$ and proportional control strategies in terms of the system norms $\left\|T_{x w}\right\|_{2},\left\|T_{x w}\right\|_{\infty}$ and $\mid T_{u w} \|_{2}$, where $T_{x w}$ is the transfer matrix from the disturbance input $w$ to the state $x$, and $T_{u w}$ is the transfer matrix from the disturbance input $w$ to the control $u$. Thus, the norms are measures of the state response to Gaussian disturbances, the state response to worst-case disturbances, 
and the control used in response to Gaussian disturbances, respectively. For case 1, the results show that the proportional controllers are not nearly as effective as the $\mathscr{H}_{2}$ and $\mathscr{H}_{\infty}$ controllers. The best $\mathscr{H}_{\infty}$ controller tested, is reported to perform better than all proportional controllers tested with respect to the response of the state to both white noise disturbances and worst case disturbances, and use significantly less control energy than the proportional controllers.

Turning now to case (2), Figure 11 shows the eleven least stable poles for this case. Clearly, they are all in the left half plane. Figure 12 shows the eigenvectors corresponding to the eleven least stable eigenvalues. The eigenvectors appear pairwise quite similar (except for the first eigenvector), implying that they are highly nonorthogonal. The problem of large transient energy growth is connected with this nonorthogonality, along with the magnitude of the corresponding eigenvalues. This can be illustrated by the following example, involving the second order time invariant system

$$
\dot{x}=A x=\left[\begin{array}{cc}
-1 & 0 \\
a & -11
\end{array}\right] x
$$

with initial condition $x(0)=x_{0}$. The eigenvalues of $A$ are $\lambda_{1}=-1$ and $\lambda_{2}=-11$, that is, they are independent of $a$ by the triangular structure of $A$. The associated normalized eigenvectors of $A$ are $v_{1}=\left[\begin{array}{ll}1 & a / 10\end{array}\right]^{T} / \sqrt{a^{2} / 100+1}$ and $v_{2}=\left[\begin{array}{ll}0 & 1\end{array}\right]^{T}$, respectively. The scalar product of the two eigenvectors is

$$
v_{1} \cdot v_{2}=\frac{a}{\sqrt{a^{2}+100}}
$$

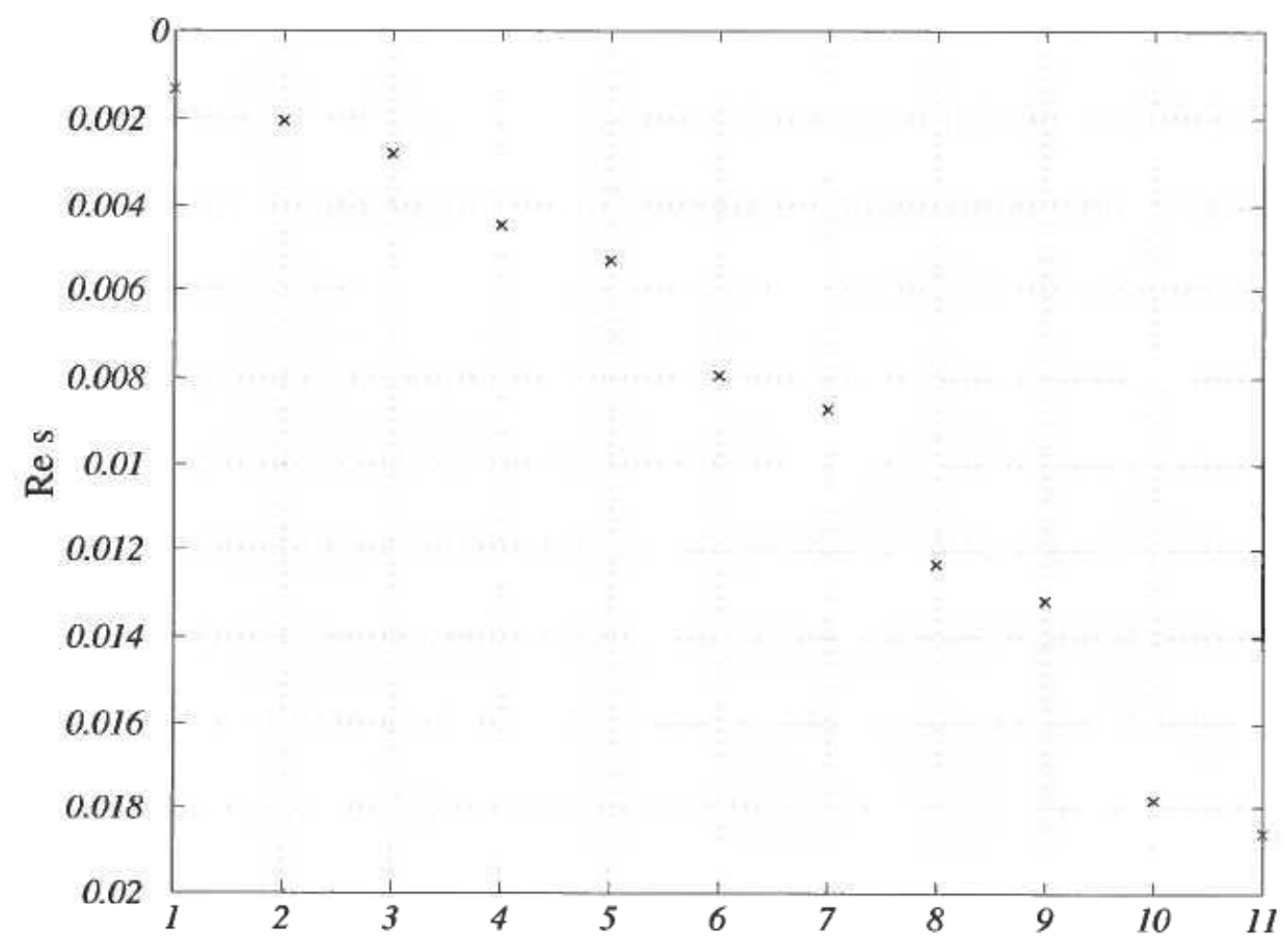

Figure 11. The eleven least stable eigenvalues in case 2. All eigenvalues are real. 


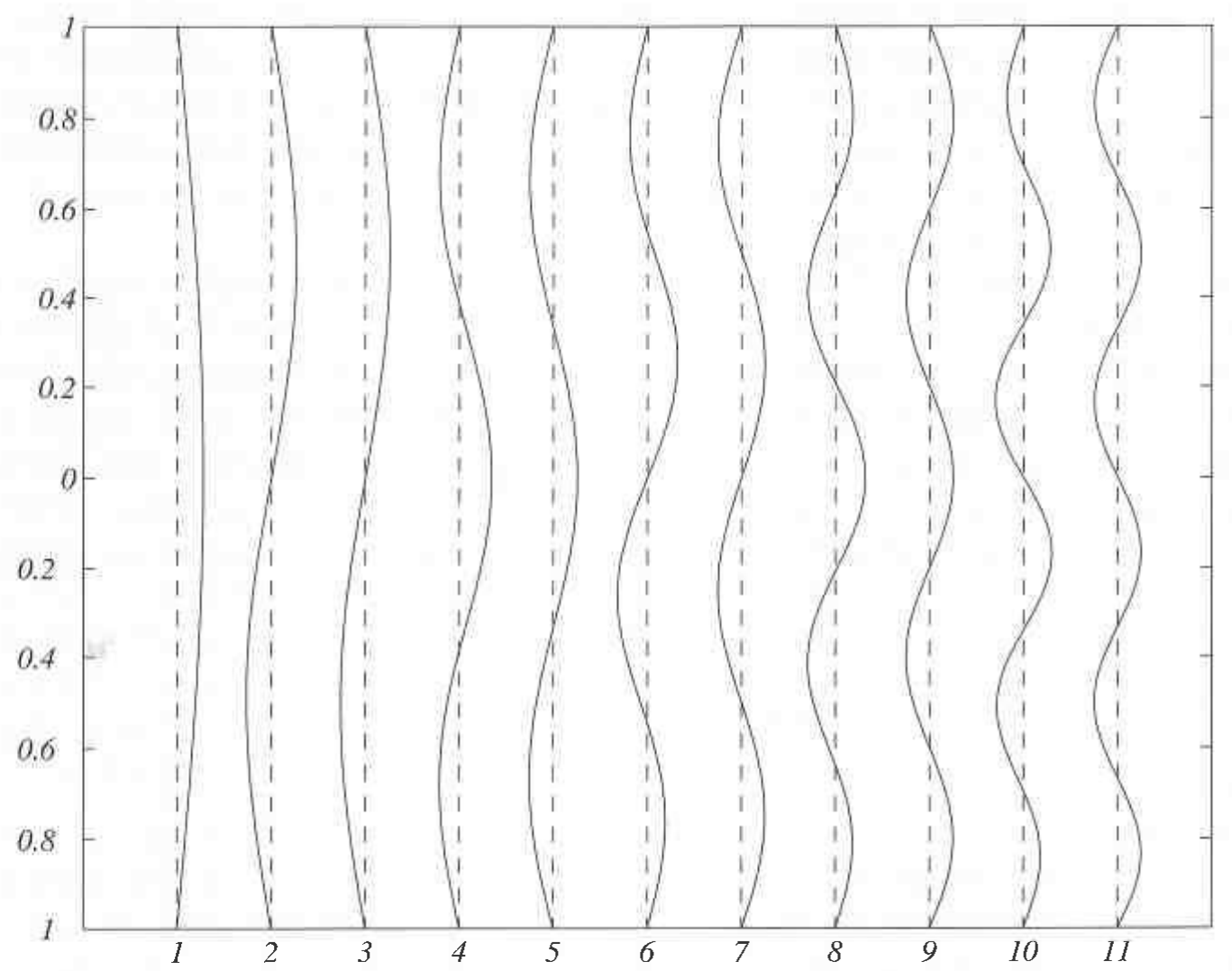

Figure 12. Eigenvectors corresponding to the eleven least stable eigenvalues.

which is maximized as $a \rightarrow \infty$. When $a=0$ the eigenvectors are orthogonal, and the energy decreases monotonically for all initial conditions, since the solution is simply given by

$$
x(t)=\left[\begin{array}{cc}
e^{-t} & 0 \\
0 & e^{-11 t}
\end{array}\right] x_{0}
$$

in this case. However, if $a=100$, for instance, the solution is given by

$$
x(t)=\left[\begin{array}{cc}
e^{-t} & 0 \\
10\left(e^{-t}-e^{-11 t}\right) & e^{-11 t}
\end{array}\right] x_{0}
$$

so the (worst case) transient energy growth is

$$
\mathscr{E}(t)=\sup _{x_{0}} \frac{\|x(t)\|_{2}}{\left\|x_{0}\right\|_{2}}=\left\|\left[\begin{array}{cc}
e^{-t} & 0 \\
10\left(e^{-t}-e^{-11 t}\right) & e^{-11 t}
\end{array}\right]\right\|_{2}=10\left(e^{-t}-e^{-11 t}\right)
$$

which is plotted in Figure 13. When this phenomenon occurs in a channel flow, transition to turbulence is likely to occur, because the nonlinear terms in the NavierStokes equation come into play. Thus, it is an important property of any control law for equations (134)-(135), that the transient energy growth is suppressed, which is the main point in Bewley and Liu (1998) regarding case (2). The parametric study carried out in Bewley and Liu (1998), indicate that the $\mathscr{H}_{2}$ and $\mathscr{H}_{\infty}$ controllers act to make the set of eigenvectors more orthogonal. The maximum transient energy growth of the system is reported to be reduced effectively. 


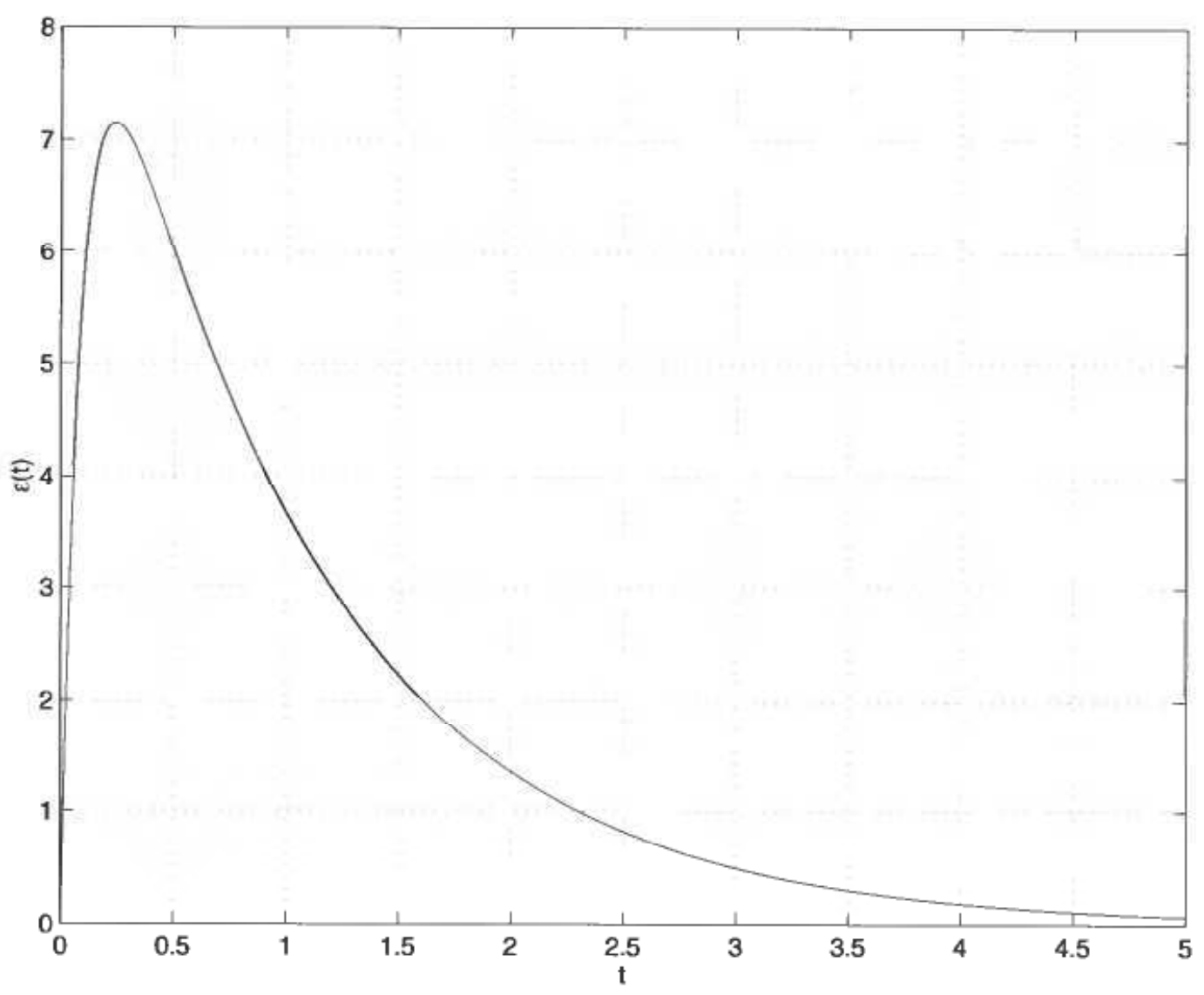

Figure 13. Energy growth in second order example system.

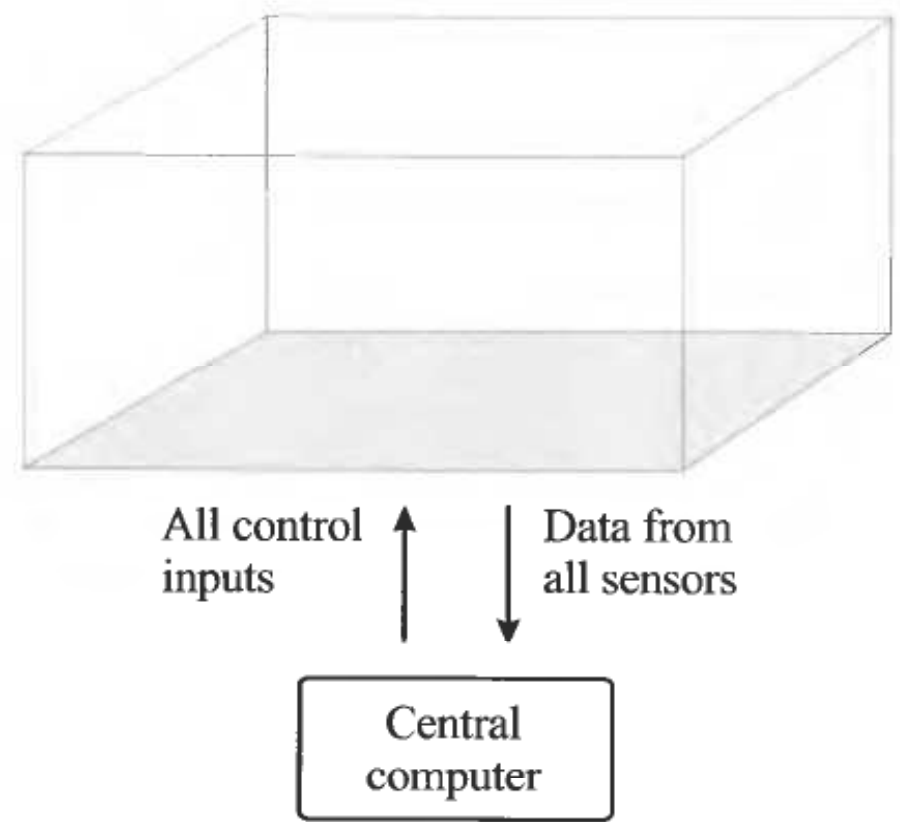

Figure 14. Centralized control. Actuators and sensors are distributed over the shaded face. All sensor data is sent to a central computer which calculates and issues control signals to all actuators.

\section{Spatial Invariance Yields Localized Control}

In Cortelezzi and Speyer (1998), an implementation of the control system sketched in Figure 6 for stabilizing the 2D chanmel flow was suggested. Figure 14 shows 
schematically the setup, involving arrays of sensors and actuators, and a central computing unit. Data from the entire sensor array is fed into the computer, Fourier transformed, and then fed to the control algorithm. The resulting control signal is inverse Fourier transformed and output to the actuator array. The communication needs are tremendous, and so is the computational load. It would be desirable to have localized control, that is, actuation at a certain spatial position should depend on sensing in a neighborhood of that location. Intuitively, flow variables far away from a certain actuation point should be less important than flow variables that are closer. And indeed, this is the case, as shown in Bamieh et al. (2000). The results are based on the notion of spatial invariance. Loosely put, the system is spatially invariant with respect to the spatial variable $x$ if the system looks the same looking up and down the $x$-axis, regardless of the point of reference. It is clear that the prototype flows studied here have this property in the streamwise (and spanwise, in the 3D case) direction. In this case, the optimal controllers derived above also are spatially invariant. Moreover, the optimal state feedback $u=K \psi$ at a location $x$ can be written as

$$
u(x, t)=\int_{\mathbb{R}} k_{s}(x-\zeta) \psi(\zeta) d \zeta
$$

where the convolution kernel $k_{s}$ decays exponentially, that is

$$
\left|k_{s}(x)\right| \leqslant M e^{-\alpha|x|}
$$

for some positive constants $M$ and $\alpha$. Similarly, an exponentially decaying convolution kernel, $k_{e}$, can be found for the estimation problem. Thus, one can approximate the integral equation (136) to any desired degree of accuracy by truncating it at some approprate $\varepsilon>0$, to obtain

$$
u(x, t)=\int_{\mathbb{R}} k_{s}(x-\zeta) \psi(\zeta) d \zeta \approx \int_{-\varepsilon}^{\varepsilon} k_{s}(x-\zeta) \psi(\zeta) d \zeta
$$

It follows that one can design decentralized controllers that are arbitrarily close to optimal. For the linearized Navier-Stokes equations for 3D channel flow, these kernels have been calculated in Högberg and Bewley (2000). Implementation of the control can be done in terms of a lattice of identical tiles incorporating sensors, actuators and computation logic, as shown schematically in Figure 15. Each tile estimates the state above itself, and the information is communicated to its neighbors. Based on gathered information, each tile calculates its control. The choice of $\varepsilon$ determines the distance over which sensor information must be communicated.

\section{Lyapunov Stability Approach}

In this section, we use Lyapunov stability analysis to show stability of the parabolic equilibrium profile equation (72), for 2D channel flow (Aamo et al., 2001; Balogh et al., 2001). The results extend easily to 3D channel flow and pipe flow as well. The Navier-Stokes equations are nonlinear, and the only way we can assure global stability of the feedback system is by nonlinear analysis. Note, though, that this does not neccessarily mean that the stabilizing feedback control law has to be nonlinear. Indeed, the result of the analysis below, turns out to give astonishingly simple control laws, that are linear and completely decentralized, and that satisfy Lyapunov's stability criterion (in $L_{2}$ norm) for nonlinear systems. 


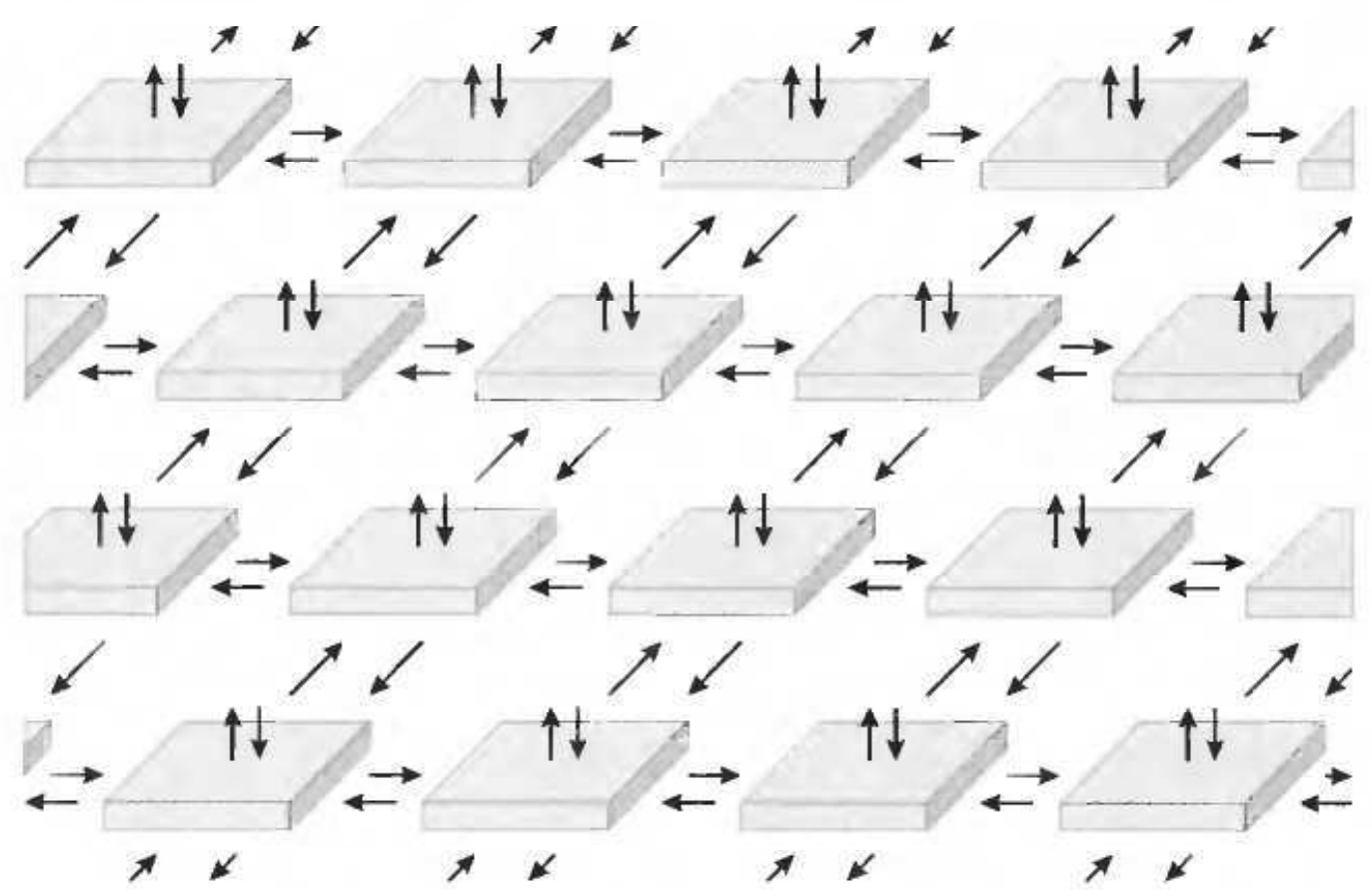

Figure 15. Decentralized control. A lattice of identical tiles incorporating sensors and actuators, and computation logic. Sensor information is communicated between neighboring tiles, and each tile computes the control above itself.

\subsection{D channel flow}

6.1.1. Lyapunov stability analysis Boundary control laws for stabilization are sought such that the kinetic energy of the system decays as a function of time. This is a standard Lyapunov-based approach, in which the Lyapunov function is chosen as

$$
E(\mathbf{w})=\|\mathbf{w}\|_{L_{2}}^{2}=\int_{-1}^{1} \int_{0}^{L}\left(u^{2}+v^{2}\right) d x d y
$$

The Lyapunov analysis is performed in the perturbation variables, since the parabolic equilibrium profile is moved to the origin in these variables. Moving the equilibrium point that is to be stabilized to the origin by means of a coordinate transformation is standard procedure in Lyapunov analysis. The time derivative of $E(\mathbf{w})$ along the trajectories of equations (74)-(75) is

$$
\begin{aligned}
\dot{E}(\mathbf{w})= & 2 \int_{-1}^{1} \int_{0}^{L}\left(u \frac{\partial u}{\partial t}+v \frac{\partial v}{\partial t}\right) d x d y \\
= & 2 \int_{-1}^{1} \int_{0}^{L} u\left(\frac{1}{R}\left(\frac{\partial^{2} u}{\partial x^{2}}+\frac{\partial^{2} u}{\partial y^{2}}\right)-u \frac{\partial u}{\partial x}-\tilde{U} \frac{\partial u}{\partial x}-v \frac{\partial u}{\partial y}-v \frac{d \tilde{U}}{d y}-\frac{\partial p}{\partial x}\right) d x d y \\
& +2 \int_{-1}^{1} \int_{0}^{L} v\left(\frac{1}{R}\left(\frac{\partial^{2} v}{\partial x^{2}}+\frac{\partial^{2} v}{\partial y^{2}}\right)-u \frac{\partial v}{\partial x}-\tilde{U} \frac{\partial v}{\partial x}-v \frac{\partial v}{\partial y}-\frac{\partial p}{\partial y}\right) d x d y
\end{aligned}
$$

Integration by parts, noticing that

$$
u^{2} \frac{\partial u}{\partial x}=\frac{1}{2} u \frac{\partial\left(u^{2}\right)}{\partial x} \quad \text { and } \quad v^{2} \frac{\partial v}{\partial y}=\frac{1}{2} v \frac{\partial\left(v^{2}\right)}{\partial y}
$$


and keeping in mind that all the variables are periodic in $x$, yields*

$$
\begin{aligned}
& \dot{E}(\mathbf{w})=\frac{2}{R} \int_{0}^{L}\left[\frac{\partial u}{\partial y} u+\frac{\partial v}{\partial y} v\right]_{y=-1}^{1} d x-\frac{2}{R} \int_{-1}^{1} \int_{0}^{L}\left(\left(\frac{\partial u}{\partial x}\right)^{2}+\left(\frac{\partial u}{\partial y}\right)^{2}+\left(\frac{\partial v}{\partial x}\right)^{2}+\left(\frac{\partial v}{\partial y}\right)^{2}\right) d x d y \\
& +\int_{-1}^{1} \int_{0}^{L} u^{2} \frac{\partial u}{\partial x} d x d y-2 \underbrace{\int_{-1}^{1} \int_{0}^{L} \tilde{U} \frac{\partial u}{\partial x} u d x d y}_{=0^{1}}-\int_{0}^{L}\left[u^{2} v\right]_{y=-1}^{1} d x \\
& +\int_{-1}^{1} \int_{0}^{L} u^{2} \frac{\partial v}{\partial y} d x d y-2 \int_{-1}^{1} \int_{0}^{L} v u \frac{d \tilde{U}}{d y} d x d y-2 \int_{-1}^{1} \int_{0}^{L} \frac{\partial u}{\partial x} p d x d y \\
& +\int_{-1}^{1} \int_{0}^{L} v^{2} \frac{\partial u}{\partial x} d x d y-2 \underbrace{\int_{-1}^{1} \int_{0}^{L} \tilde{U} \frac{\partial v}{\partial x} v d x d y}_{=0^{1}}-\int_{0}^{L}\left[v^{3}\right]_{y=-1}^{1} d x \\
& +\int_{-1}^{1} \int_{0}^{L} v^{2} \frac{\partial v}{\partial y} d x d y-2 \int_{0}^{L}[v p]_{y=-1}^{1} d x+2 \int_{-1}^{1} \int_{0}^{L} \frac{\partial v}{\partial y} p d x d y \\
& =-\frac{2}{R} \int_{-1}^{1} \int_{0}^{L}\left(\left(\frac{\partial u}{\partial x}\right)^{2}+\left(\frac{\partial u}{\partial y}\right)^{2}+\left(\frac{\partial v}{\partial x}\right)^{2}+\left(\frac{\partial v}{\partial y}\right)^{2}\right) d x d y-2 \int_{-1}^{1} \int_{0}^{L} v u \frac{d \tilde{U}}{d y} d x d y \\
& +\int_{-1}^{1} \int_{0}^{L} u^{2}\left(\frac{\partial u}{\partial x}+\frac{\partial v}{\partial y}\right) d x d y-2 \int_{-1}^{1} \int_{0}^{L} p\left(\frac{\partial u}{\partial x}+\frac{\partial v}{\partial y}\right) d x d y \\
& +\int_{-1}^{1} \int_{0}^{L} v^{2}\left(\frac{\partial u}{\partial x}+\frac{\partial v}{\partial y}\right) d x d y+\frac{2}{R} \int_{0}^{L}\left[\frac{\partial u}{\partial y} u\right]_{y=-1}^{1} d x \\
& +\frac{2}{R} \int_{0}^{L}\left[\left(\frac{\partial v}{\partial y}-R p\right) v\right]_{y=-1}^{1} d x-\int_{0}^{L}\left[\left(u^{2}+v^{2}\right) v\right]_{y=-1}^{1} d x
\end{aligned}
$$

Using continuity equation (73), we get

$$
\begin{aligned}
\dot{E}(\mathbf{w})= & -\frac{2}{R} \int_{-1}^{1} \int_{0}^{L} \int_{0}^{L}\left(\left(\frac{\partial u}{\partial x}\right)^{2}+\left(\frac{\partial u}{\partial y}\right)^{2}+\left(\frac{\partial v}{\partial x}\right)^{2}+\left(\frac{\partial v}{\partial y}\right)^{2}\right) d x d y \\
& -2 \int_{-1}^{1} \int_{0}^{L} u v \frac{d \tilde{U}}{d y} d x d y+\frac{2}{R} \int_{0}^{L}\left[\frac{\partial u}{\partial y} u\right]_{y=-1}^{1} d x \\
& +\frac{2}{R} \int_{0}^{L}\left[\left(\frac{\partial v}{\partial y}-R p\right) v\right]_{y=-1}^{1} d x-\int_{0}^{L}\left[\left(u^{2}+v^{2}\right) v\right]_{y=-1}^{1} d x
\end{aligned}
$$

* Due to periodic boundary conditions in the streamwise direction:

$$
\int_{0}^{L} \tilde{U} \frac{\partial u}{\partial x} u d x=\frac{\tilde{U}}{2} \int_{0}^{L} \frac{\partial\left(u^{2}\right)}{\partial x} d x=\frac{\tilde{U}}{2}\left[u^{2}\right]_{0}^{L}=0 .
$$


Following Balogh et al. (2001) Lemma 6.2 (also in Balogh et al. (1999) Lemma 3.2), we set

$$
u(x, y, t)=u(x,-1, t)+\int_{-1}^{y} \frac{\partial u}{\partial y}(x, \gamma, t) d \gamma
$$

where the integration variable is denoted $\gamma$ for notational clearity. Squaring equation (141) yields

$$
u^{2}(x, y, t)=\left(u(x,-1, t)+\int_{-1}^{y} \frac{\partial u}{\partial y}(x, \gamma, t) d \gamma\right)^{2} \leqslant 2 u^{2}(x,-1, t)+2\left(\int_{-1}^{y} \frac{\partial u}{\partial y}(x, \gamma, t) d \gamma\right)^{2}
$$

By the Schwartz inequality,

$$
\left(\int_{-1}^{y} 1 \frac{\partial u}{\partial y}(x, \gamma, t) d \gamma\right)^{2} \leqslant(y+1)\left(\int_{-1}^{y}\left(\frac{\partial u}{\partial y}(x, \gamma, t)\right)^{2} d \gamma\right)
$$

so we have that

$$
u^{2}(x, y, t) \leqslant 2 u^{2}(x,-1, t)+2(y+1) \int_{-1}^{1}\left(\frac{\partial u}{\partial y}(x, y, t)\right)^{2} d y
$$

where we have set $y=1$ in the integral. Therefore, we get

$$
\begin{aligned}
\int_{-1}^{1} \int_{0}^{L} u^{2} d x d y \leqslant & 4 \int_{0}^{L} u^{2}(x,-1, t) d x d y \\
& +2 \int_{-1}^{1} \int_{0}^{L}(y+1)\left(\int_{-1}^{1}\left(\frac{\partial u}{\partial y}(x, y, t)\right)^{2} d y\right) d x d y \\
= & 4 \int_{0}^{L} u^{2}(x,-1, t) d x d y+2 \int_{0}^{L}\left(\int_{-1}^{1}(y+1) d y\right) \\
& \left(\int_{-1}^{1}\left(\frac{\partial u}{\partial y}(x, y, t)\right)^{2} d y\right) d x \\
= & 4 \int_{0}^{L} u^{2}(x,-1, t) d x d y+4 \int_{-1}^{1} \int_{0}^{L}\left(\frac{\partial u}{\partial y}(x, y, t)\right)^{2} d x d y
\end{aligned}
$$

An anologous derivation for $(\partial v / \partial y)$ now gives

$$
-\int_{-1}^{1} \int_{0}^{L}\left(\left(\frac{\partial u}{\partial y}\right)^{2}+\left(\frac{\partial v}{\partial y}\right)^{2}\right) d x d y \leqslant-\frac{E(\mathbf{w})}{4}+\int_{0}^{L}\left(u^{2}(x,-1, t)+v^{2}(x,-1, t)\right) d x
$$


Inserting equation (145) into (140) we get

$$
\begin{aligned}
\dot{E}(\mathbf{w}) \leqslant & -\frac{1}{2 R} E(\mathbf{w})+\frac{2}{R} \int_{0}^{L}\left(u^{2}(x,-1, t)+v^{2}(x,-1, t)\right) d x \\
& -\frac{2}{R} \int_{-1}^{1} \int_{0}^{L}\left(\left(\frac{\partial u}{\partial x}\right)^{2}+\left(\frac{\partial v}{\partial x}\right)^{2}\right) d x d y-2 \int_{-1}^{1} \int_{0}^{L} u v \frac{d \tilde{U}}{d y} d x d y \\
& +\frac{2}{R} \int_{0}^{L}\left[\frac{\partial u}{\partial y} u\right]_{y=-1}^{1} d x+\frac{2}{R} \int_{0}^{L}\left[\left(\frac{\partial v}{\partial y}-R p\right) v\right]_{y=-1}^{1} d x \\
& -\int_{0}^{L}\left[\left(u^{2}+v^{2}\right) v\right]_{y=-1}^{1} d x
\end{aligned}
$$

Since

$$
-2 \int_{-1}^{1} \int_{0}^{L} u v \frac{d \tilde{U}}{d y} d x d y \leqslant 2 \int_{-1}^{1} \int_{0}^{L} 2|u||v| d x d y \leqslant 2 \int_{-1}^{1} \int_{0}^{L}\left(u^{2}+v^{2}\right) d x d y=2 E(\mathbf{w})
$$

we finally get

$$
\begin{aligned}
\dot{E}(\mathbf{w}) \leqslant & -\frac{1}{2}\left(\frac{1}{R}-4\right) E(\mathbf{w})+\frac{2}{R} \int_{0}^{L}\left(u^{2}(x,-1, t)+v^{2}(x,-1, t)\right) d x+\frac{2}{R} \int_{0}^{L}\left[\frac{\partial u}{\partial y} u\right]_{y=-1}^{1} d x \\
& +2 \int_{0}^{L}\left[\left(\frac{1}{R} \frac{\partial v}{\partial y}-p\right) v\right]_{y=-1}^{1} d x-\int_{0}^{L}\left[\left(u^{2}+v^{2}\right) v\right]_{y=-1}^{1} d x
\end{aligned}
$$

Notice that for $R<1 / 4, E(\mathbf{w})$ decays exponentially with time even in the uncontrolled case $(u(x, \pm 1, t)=v(x, \pm 1, t) \equiv 0)$. In other words, the fixed point $(\tilde{U}, \tilde{V})$ is globally exponentially stable (in $L_{2}$ ) in this case, and the goal of applying boundary control is to enhance stability. The four last terms in equation (148) are evaluated on the boundary, and are the means by which boundary control laws are designed.

Below, two control laws are presented: the first uses wall-tangential actuation; and the second uses wall-normal actuation.

6.1.2. Wall-tangential distributed actuation The following boundary control was suggested in Balogh et al. (2001):

$$
\begin{aligned}
& u(x,-1, t)=k_{u} \frac{\partial u}{\partial y}(x,-1, t), u(x, 1, t)=-k_{u} \frac{\partial u}{\partial y}(x, 1, t) \\
& v(x,-1, t)=v(x, 1, t)=0
\end{aligned}
$$

Inserting equations (149)-(150) into (148) gives

$$
\dot{E}(\mathbf{w}) \leqslant-\frac{1}{2}\left(\frac{1}{R}-4\right) E(\mathbf{w})-\frac{2}{R}\left(\frac{1}{k_{u}}-1\right) \int_{0}^{L} u^{2}(x,-1, t) d x
$$

Thus, for $R<1 / 4$ and $k_{u} \in[0,1], E(\mathbf{w})$ decays exponentially with time. 
6.1.3. Wall-normal distributed actuation Actuation normal to the wall is another strategy of active interest. The inequality equation (148) also suggests a control law structure for wall-normal control (Aamo et al., 2001). Setting $u(x,-1, t)=$ $u(x, 1, t)=0,(\partial v / \partial y)$ is zero at the wall by continuity equation (73), so we have

$$
\dot{E}(\mathbf{w}) \leqslant-\frac{1}{2}\left(\frac{1}{R}-4\right) E(\mathbf{w})+\frac{2}{R} \int_{0}^{L} v^{2}(x,-1, t) d x-2 \int_{0}^{L}[p v]_{y=-1}^{1} d x-\int_{0}^{L}\left[v^{3}\right]_{y=-1}^{1} d x
$$

Now, by imposing $v(x,-1, t)=v(x, 1, t)$, the last term in equation (152) vanishes. Thus, we propose the following control law

$$
\begin{aligned}
& u(x,-1, t)=u(x, 1, t)=0 \\
& v(x,-1, t)=v(x, 1, t)=k_{v}(p(x, 1, t)-p(x,-1, t))
\end{aligned}
$$

Inserting equations (153)-(154) into (152) gives

$$
\dot{E}(\mathbf{w}) \leqslant-\frac{1}{2}\left(\frac{1}{R}-4\right) E(\mathbf{w})-2\left(\frac{1}{k_{v}}-\frac{1}{R}\right) \int_{0}^{L} v^{2}(x,-1, t) d x
$$

Thus, for $R<1 / 4$ and $k_{v} \in[0, R], E(\mathbf{w})$ decays exponentially with time. Furthermore, note that equation (154) ensures that the net mass flux through the walls be zero.

6.1.4. Implementation In order to implement the above controllers we have to express them in terms of the actual flow variables, $U, V$ and $P$. For the wall-tangential case, we get

$$
\begin{aligned}
U(x,-1, t) & =k_{u}\left(\frac{\partial U}{\partial y}(x,-1, t)-\frac{d \tilde{U}}{d y}(x,-1, t)\right) \\
U(x, 1, t) & =-k_{u}\left(\frac{\partial U}{\partial y}(x, 1, t)-\frac{d \tilde{U}}{d y}(x, 1, t)\right) \\
V(x,-1, t) & =V(x, 1, t)=0
\end{aligned}
$$

and for the wall-normal case we get

$$
\begin{aligned}
& U(x,-1, t)=U(x, 1, t)=0 \\
& V(x,-1, t)=V(x, 1, t)=k_{v}(P(x, 1, t)-P(x,-1, t))
\end{aligned}
$$

It is interesting to notice that the wall-normal control is independent of the physical parameters of the flow. This is an important property, since the physical parameters of any real flow are subject to inaccuracy. In contrast, $(d \tilde{U} / d y)(x, \pm 1, t)$ must be known for wall-tangential control.

It is also worth noting that all the above control laws are of the Jurdjevic-Quinn (Jurdjevic and Quinn, 1978) type (with respect to the Lyapunov function $E(w)$ ). This endows these control laws with inverse optimality with respect to a meaningful cost functional (which is in these cases complicated to write).

6.1.5. Numerical demonstration The theoretical results of Section 6.1.1 are only valid for Reynolds numbers less than $1 / 4$, for which the parabolic equilibrium profile is globally exponentially stable in the uncontrolled case. Thus, the analysis only tells us that the proposed control laws maintain stability, and not neccessarily enhance it. In 
fact, for wall-normal control, simulations at $R=0.1$ show that for $k_{v}=0.1, E(\mathbf{w})$ converges more slowly to 0 than in the uncontrolled case, whereas for $k_{v}=-0.1$, stability is enhanced. Although this result was unexpected, it does not contradict the theoretical results.

Being valid for small Reynolds numbers only, the theoretical results are of limited practical value. However, they do suggest controller structures worth testing on flows having higher Reynolds numbers. In Balogh et al. (2001), results from numerical simulations with wall-tangential control were presented that show stabilization of channel flow at $R=15000$. Here, we do a comparison of the performance of the two control laws for flows at $R=7500$ and $L=4 \pi$.

The simulations are performed using a hybrid Fourier pseudospectral-finite difference discretization and the fractional step technique based on a hybrid RungeKutta/Crank-Nicolson time discretization using the numerical method of Bewley and Moin (1999). This method is particularly well suited even for the cases with wallnormal actuation because of its implicit treatment of the wall-normal convective terms. The wall-paralel direction is discretized using 128 Fourier-modes, while the wall-normal direction is discretized using energy-conserving central finite differences on a stretched staggered grid with 100 gridpoints. The gridpoints have hyperbolic tangent distribution in the wall-normal direction in order to adequately resolve the high-shear regions near the walls. A fixed flow-rate formulation is used, rather than fixed average pressure gradient, since observations suggest that the approach to equilibrium is faster in this case (Jiménez, 1990). The difference between the two formulations is discussed briefly in Rozhdestvensky and Simakin (1984). The time step is in the range 0.05-0.07 for all simulations. In addition to reporting the time evolution of the energy, $E(\mathbf{w})$, we also consider the (instantaneous) control effort and drag force as measures of performance. The control effort is defined as

$$
C(\mathbf{w})=\sqrt{\int_{0}^{L}\left(|\mathbf{w}(x,-1, t)|^{2}+|\mathbf{w}(x, 1, t)|^{2}\right) d x}
$$

and the drag force as

$$
D(\mathbf{w})=\frac{1}{L} \int_{0}^{L}\left(\frac{\partial U}{\partial y}(x,-1, t)-\frac{\partial U}{\partial y}(x, 1, t)\right) d x
$$

(Notice that equation (161) is really the mean wall shear, which is related to the drag force by the factor $\mu L$ ). For selected time instants, vorticity maps are also provided. The vorticity, $\omega$, is defined using the actual flow variables (rather than the perturbation variables) as

$$
\omega(x, y, t)=\frac{\partial V}{\partial x}(x, y, t)-\frac{\partial U}{\partial y}(x, y, t)
$$

A total of six simulations are performed: wall-tangential control with $k_{u} \in[0.05,0.1,0.2]$; and wall-normal control with $k_{v} \in[-0.125,-0.08,-0.05]$. The parabolic equilibrium profile is unstable for $R=7500$, so infinitesimal disturbances will grow, but the flow eventually reaches a statistically steady state, which we call fully established flow. For all simulations, the fully established flow, for which $E(\mathbf{w}) \approx 1.3$, is chosen as the initial data. Figure 16 shows a vorticity map for the fully established (uncontrolled) flow. It is similar to vorticity maps presented in Jiménez (1990), and clearly shows the ejection of vorticity from the walls into the core of the channel as described in Jiménez (1990). 


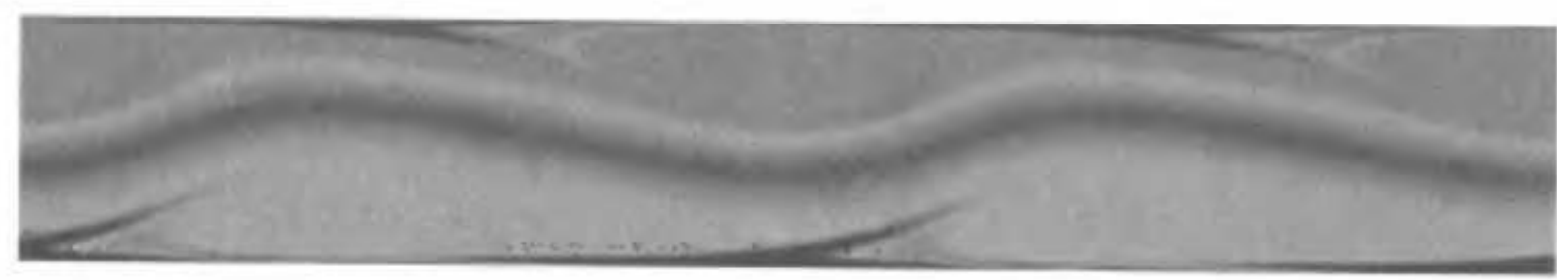

Figure 16. Vorticity map for the fully established 2D channel flow (uncontrolled).

Figure 17 compares wall-tangential and wall-normal control. It is clear that stabilization is obtained for both controllers, in terms of the energy $E(w)$. Figure 17 shows that $E(\mathbf{w})$ decays faster for wall-normal control, and at much less control effort (notice the different scales for $C(w)$ for the two cases in Figure 17). The ratio of the peak kinetic energy of the control flow (wall-normal), versus the perturbation kinetic energy in the uncontrolled case (drained out by the control), $C(w)^{2} / E(w)$, is less than $0.25 \%$. Also, reduction of drag is more efficient in the wall-normal control case.*

Figure 18 shows vorticity maps at three different time instances for wall-normal control with $k_{v}=-0.125$. The removal of vortical structures is evident already at $t=30$ (top graph), and at $t=120$ (bottom graph) the flow is nearly uniform. Figure 19 shows the pressure field immediately after onset of wall-normal control $\left(k_{v}=-0.125\right)$. Regions of low pressure coincide with regions of circulation cells, as the velocity vectors in the intermediate zoom show. In the most detailed zoom, we see that the controller applies suction in this region.

\section{2. $3 D$ channel flow}

It is recognized that channel flow instability mechanisms are inherently 3D. Efforts that study the stabilization problem only in 2D are thus inconclusive about physical flows, for which 3D effects are quite significant. However, the 'model problem' of 2D channel flow stabilization is a useful testbed for techniques that can eventually be extended to 3D flows. The Lyapunov stability analysis presented in the previous section can be extended to the 3D channel flow in a straight forward manner, and similar boundary control laws can be derived. Balogh (private communication) reports that, in numerical simulations, the very simple, and fully decentralized boundary control law

$$
\begin{aligned}
U(x,-1, z, t) & =k\left(\frac{\partial U}{\partial y}(x,-1, z, t)-\frac{\partial \tilde{U}}{\partial y}(-1)\right) \\
U(x, 1, z, t) & =-k\left(\frac{\partial U}{\partial y}(x, 1, z, t)-\frac{\partial \tilde{U}}{\partial y}(1)\right) \\
V(x,-1, z, t) & =V(x, 1, z, t)=0 \\
W(x,-1, z, t) & =W(x, 1, z, t)=0
\end{aligned}
$$

*It is interesting to note (see Figure 17) that, when the control is applied to the 2D flow, a transient ensues in which the drag dips below the laminar level and then asymptotes towards the laminar state. This transient, however, is dependent on the initial flow state being that of the fully established 2D flow, which has a drag which is significantly higher than laminar. Thus, this transient result does not disprove the conjecture stated in Bewley (2001), as discussed in Section 7. 
Wall-tangential actuation
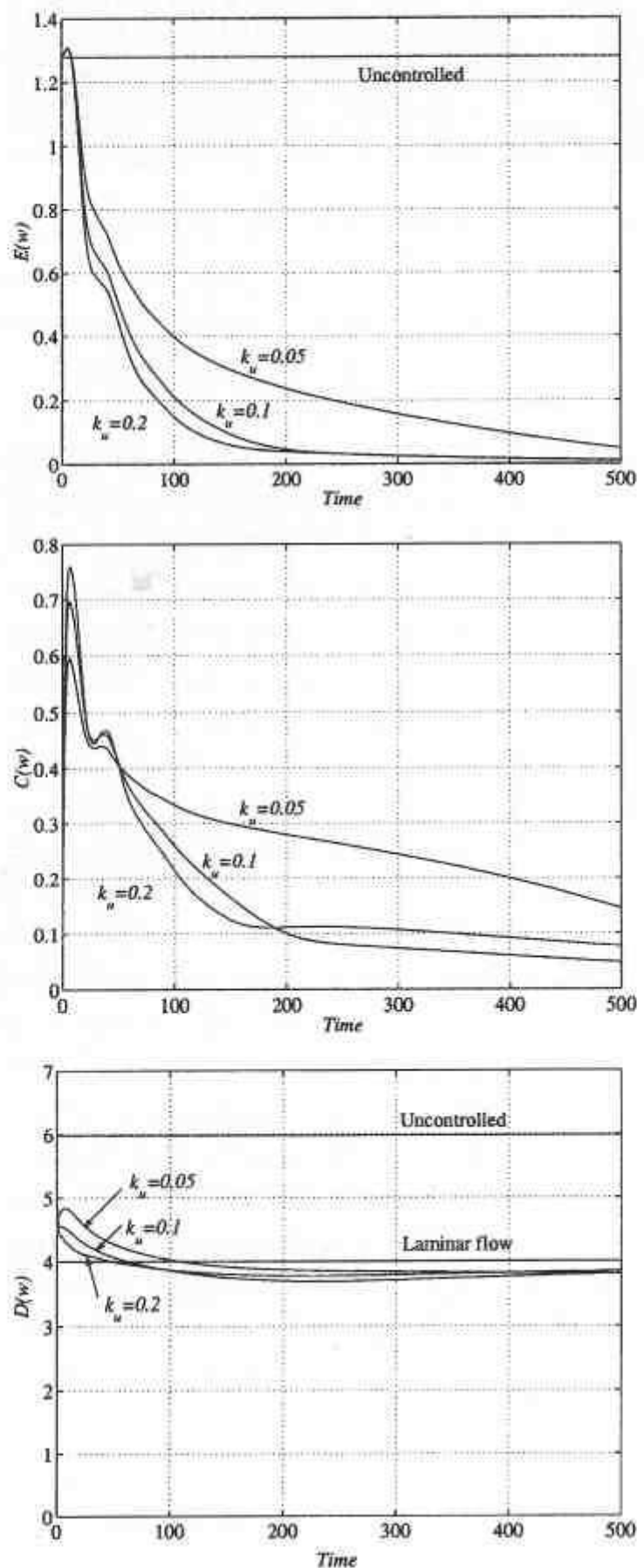

Wall-normal actuation
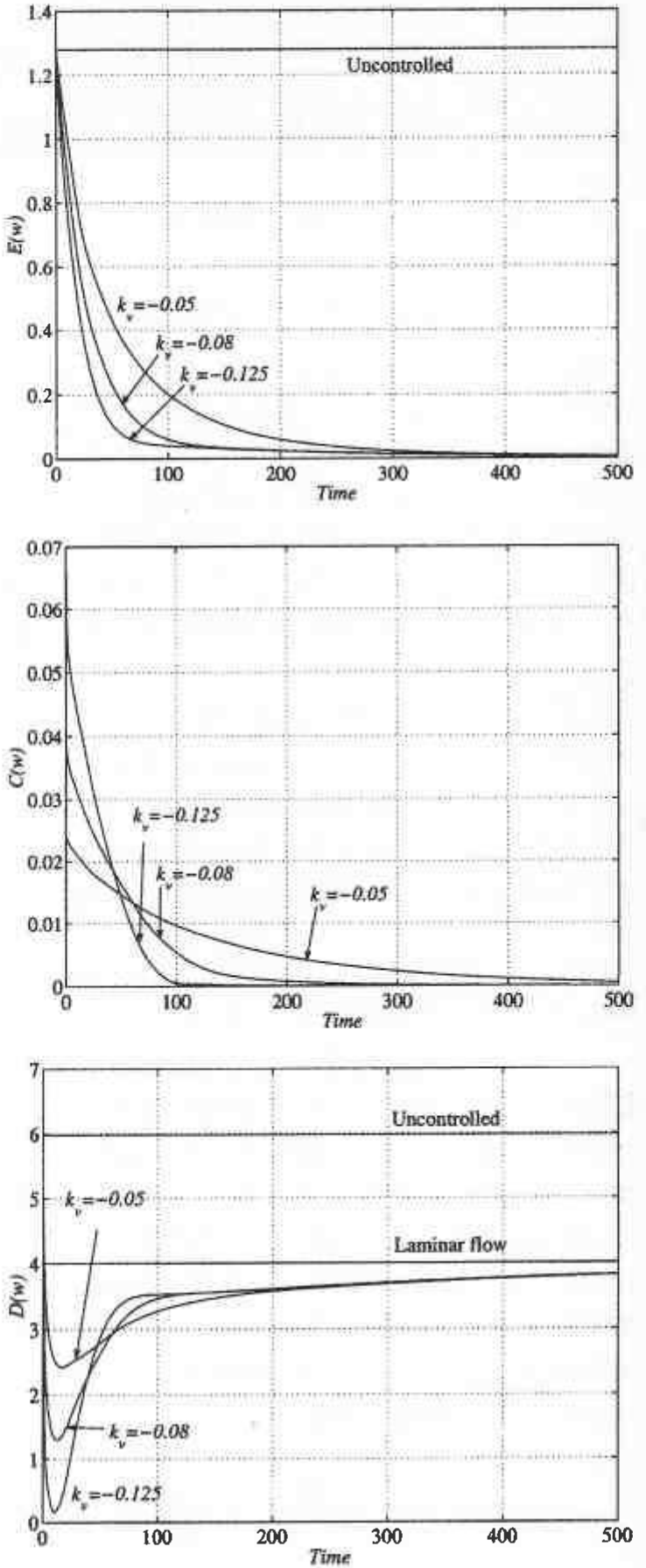

Figure 17. Energy $E(\mathbf{w})$ (top row), control effort $C(\mathbf{w})$, and drag $D(\mathbf{w})$ (bottom row), as functions of time for wall-tangential actuation (left column) and wall-normal actuation (right column).

with $k>0$, relaminarizes a turbulent 3D channel flow at $R e=4000$. Figure 20 shows the perturbation energy as a function of time. The perturbation energy is in this case

$$
E(\mathbf{w})=\int_{0}^{L_{z}} \int_{-1}^{1} \int_{0}^{L_{x}}\left(u^{2}+v^{2}+w^{2}\right) d x d y d z
$$



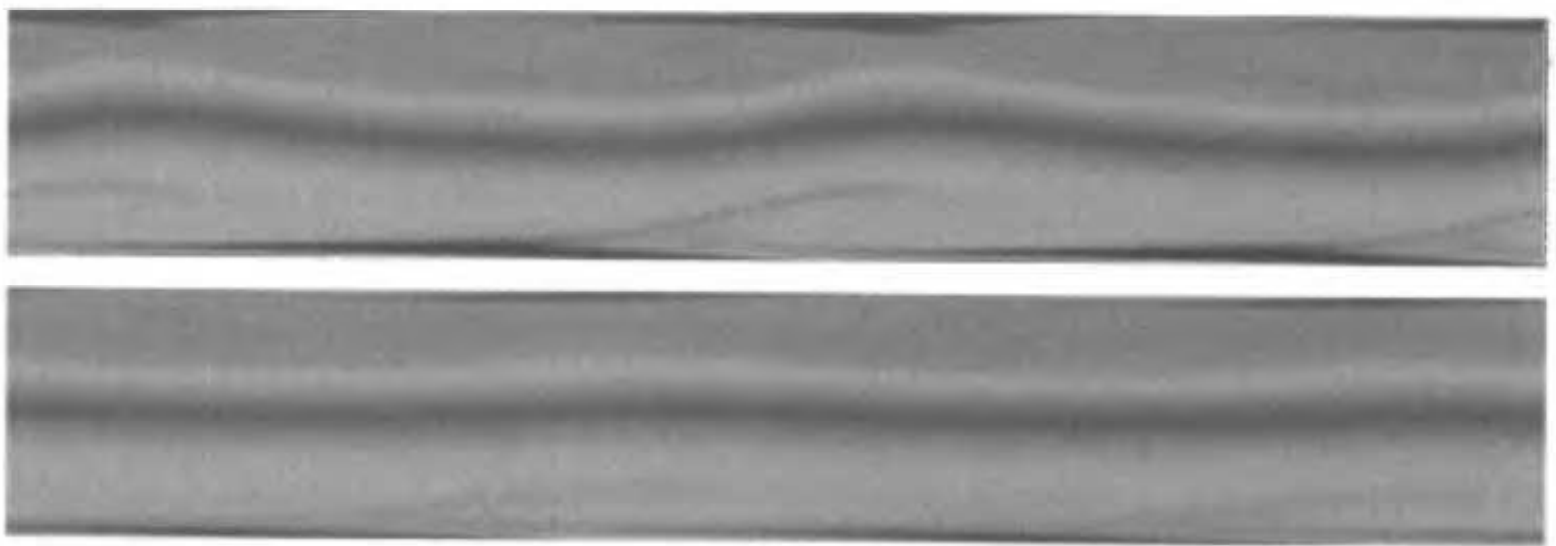

Figure 18. Vorticity maps for wall-normal actuation at $t=30$ (top figure), $t=60$, and $t=120$ (bottom figure). The feedback gain is $k_{v}=-0.125$.

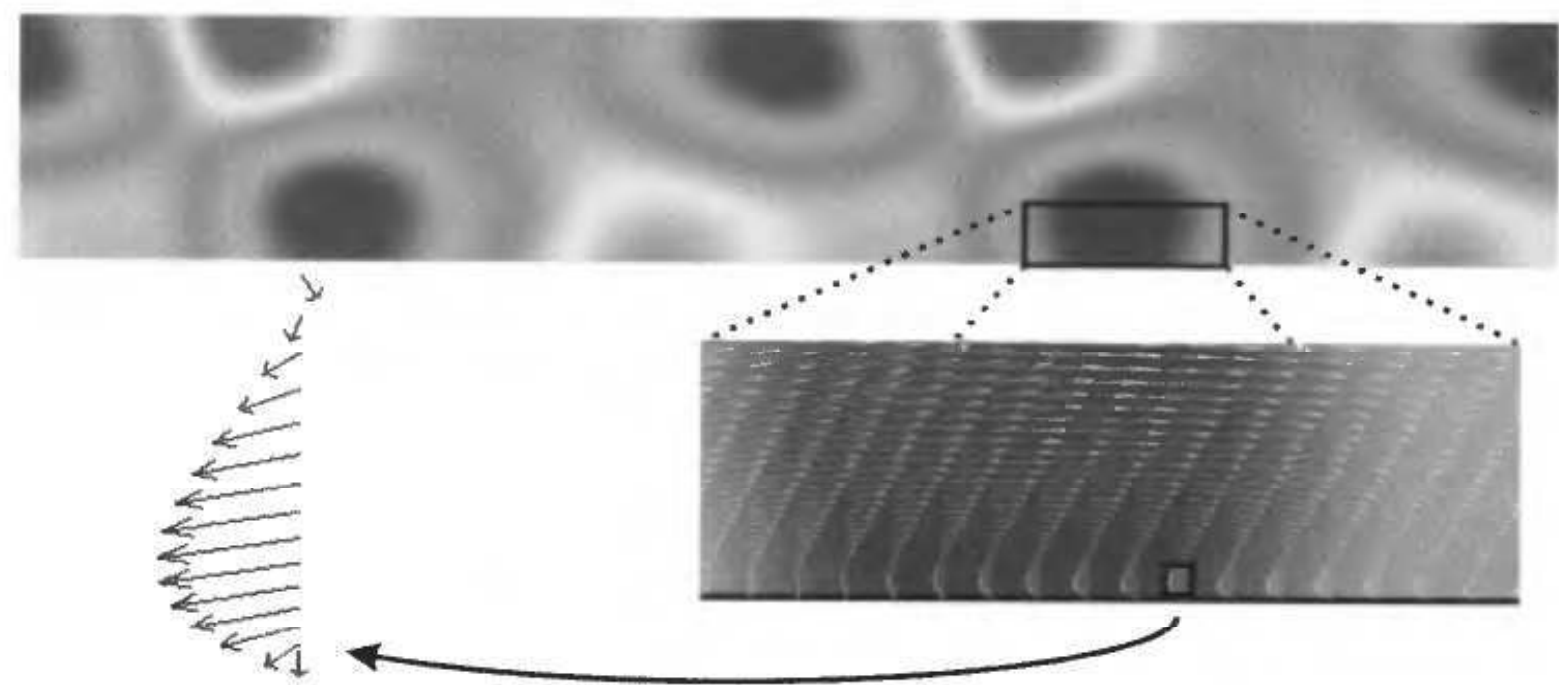

Figure 19. Pressure (perturbation only, i.e. $p$ ) immediately after onset of wall-normal actuation. Zoom shows velocity vectors in a region with low pressure.

and the channel dimensions were $L_{x}=6 \pi$ and $L_{z}=3 \pi$. The plots in Figure 21 show places where the discriminant* has values larger than 0.9 at $t=4000$, indicating locations of vortical structures. It is interesting to notice that the perturbation energy is still large at this point in time (see Figure 20), but the control has clearly influenced the vortical structures in the flow. Figure 22 shows the spanwise vorticity at $t=5000$.

* The discriminant of the velocity gradient tensor is a scalar quantity that is commonly used in visualizations to pinpoint vortex-type motions in the flow. The following definition of the discrimant is taken from Bewley et al. (2000): $D=(27 / 4) R^{2}-Q^{3}$, where $Q$ and $R$ are the second and third invariants of the velocity gradient tensor $A$, defined by $Q=\frac{1}{2}\left\{\operatorname{trace}(A)^{2}-\operatorname{trace}\left(A^{2}\right)\right\}$, and $R=-\operatorname{det}(A)$, respectively. 


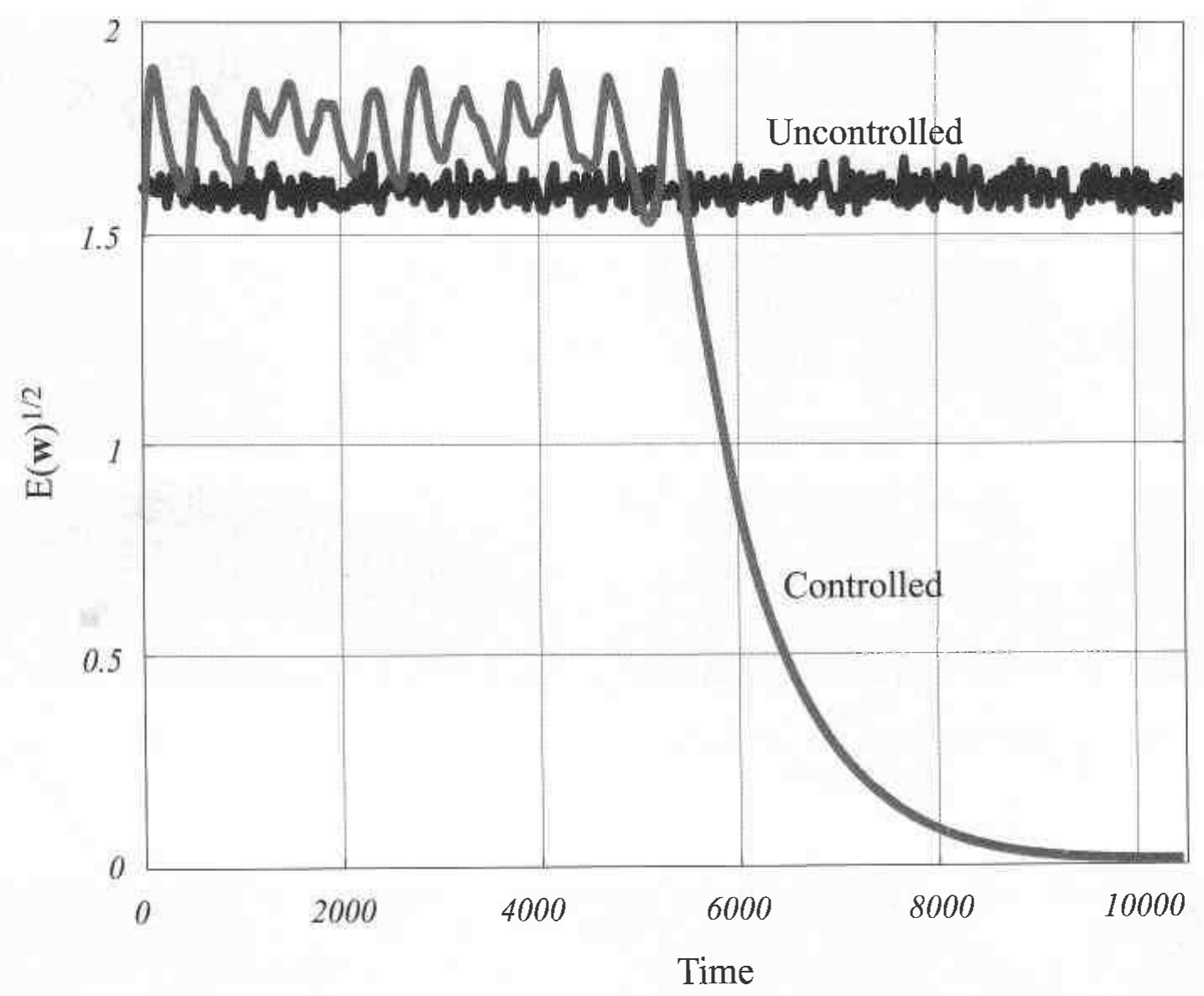

Figure 20. Perturbation energy (square root of) as a function of time for uncontrolled and $\%$ controlled 3D channel flow at $R e=4000$.

Figure 23 shows drag as a function of time. Drag is reduced to below laminar level (which is 4) almost instantly, and then gradually approaches the laminar level. This is the same result as obtained in the 2D case. Notice that drag reduction is not explicitly the objective of the control, stabilization of the parabolic equilibrium profile is. Reduction of drag to laminar level is therefore expected in the limit as $t \rightarrow \infty$, but the striking result of immediate drag reduction is obtained!

\section{3. $3 D$ pipe flow}

\subsubsection{Lyapunov stability analysis}

The Lyapunov analysis that was developed for 2D channel flow, can be modified to apply for the 3D pipe flow as well. Considering the following Lyapunov function candidate

$$
E(\mathbf{w})=\frac{1}{2}\|\mathbf{w}\|_{L 2}^{2}=\frac{1}{2} \int_{0}^{L} \int_{0}^{2 \pi} \int_{z}^{1}\left(v_{r}^{2}+v_{\theta}^{2}+v_{z}^{2}\right) r d r d \theta d z
$$

which is simply the kinetic energy of the flow, its time derivative along trajectories of equations (65)-(67) is 

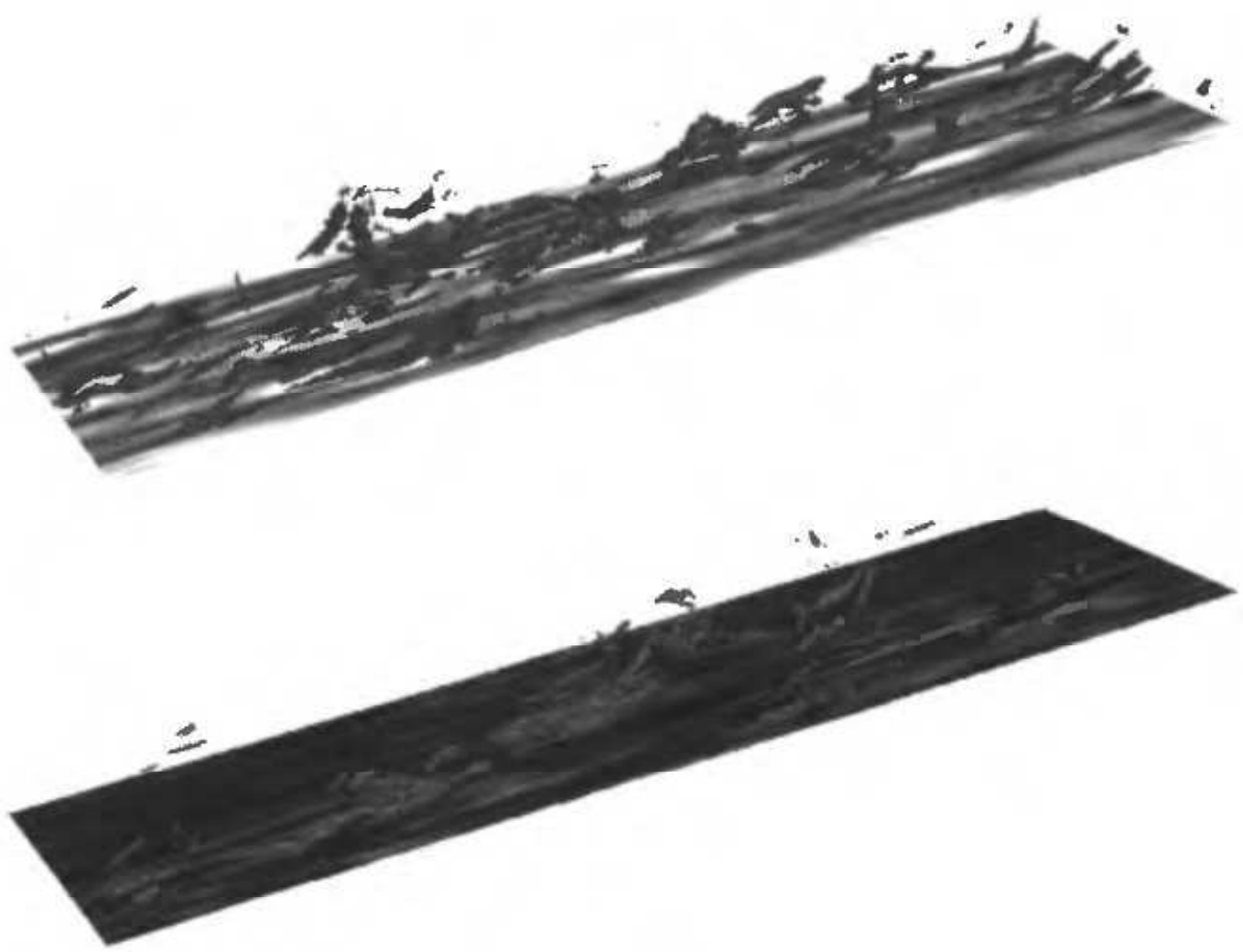

Figure 21. Places where the discriminant has values larger than 0.9 at $t=4000$, for uncontrolled (upper) and controlled (lower) 3D channel flow at $R e=4000$.

$$
\begin{aligned}
\dot{E}(w) \leqslant & 2\left(\begin{array}{c}
2 \\
R e
\end{array}\right) E(w)+\frac{2}{R e} \int_{0}^{L} \int_{0}^{2 \pi}\left[v_{r}^{2}+v_{\theta}^{2}+v_{z}^{2}\right]_{r=1} d \theta d z \\
& -\frac{1}{2} \int_{0}^{L} \int_{0}^{2 \pi}\left[v_{r}^{3}\right]_{r=1} d \theta d z-\int_{0}^{L} \int_{0}^{2 \pi}\left[v_{r} p\right]_{r=1} d \theta d z-\frac{1}{2} \int_{0}^{L} \int_{0}^{2 \pi}\left[v_{r} v_{\theta}^{2}\right]_{r=1} d \theta d z \\
& -\frac{1}{2} \int_{0}^{L} \int_{0}^{2 \pi}\left[v_{z}^{2} v_{r}\right]_{r=1} d \theta d z+\frac{1}{R e} \int_{0}^{L} \int_{0}^{2 \pi}\left[v_{r} \frac{\partial v_{r}}{\partial r}\right]_{r=1} d \theta d z \\
& +\frac{1}{\operatorname{Re}} \int_{0}^{L} \int_{0}^{2 \pi}\left[v_{\theta} \frac{\partial v_{\theta}}{\partial r}\right]_{r=1} d \theta d z+\frac{1}{\operatorname{Re}} \int_{0}^{L} \int_{0}^{2 \pi}\left[v_{z} \frac{\partial v_{z}}{\partial r}\right]_{r=1} d \theta d z
\end{aligned}
$$

In equation (163), all but the first term on the right hand side of the inequality are evaluated on the boundary. These are the terms by which boundary control laws are designed. Below, a pressure-based control law is presented.

\subsubsection{Boundary control We propose the following control law}

$$
\begin{aligned}
& v_{r}(1, \theta, z, t)=k(p(1, \theta, z, t)-p(1, \theta+\pi, z, t)) \\
& v_{\theta}(1, \theta, z, t)=0 \\
& v_{z}(1, \theta, z, t)=0
\end{aligned}
$$



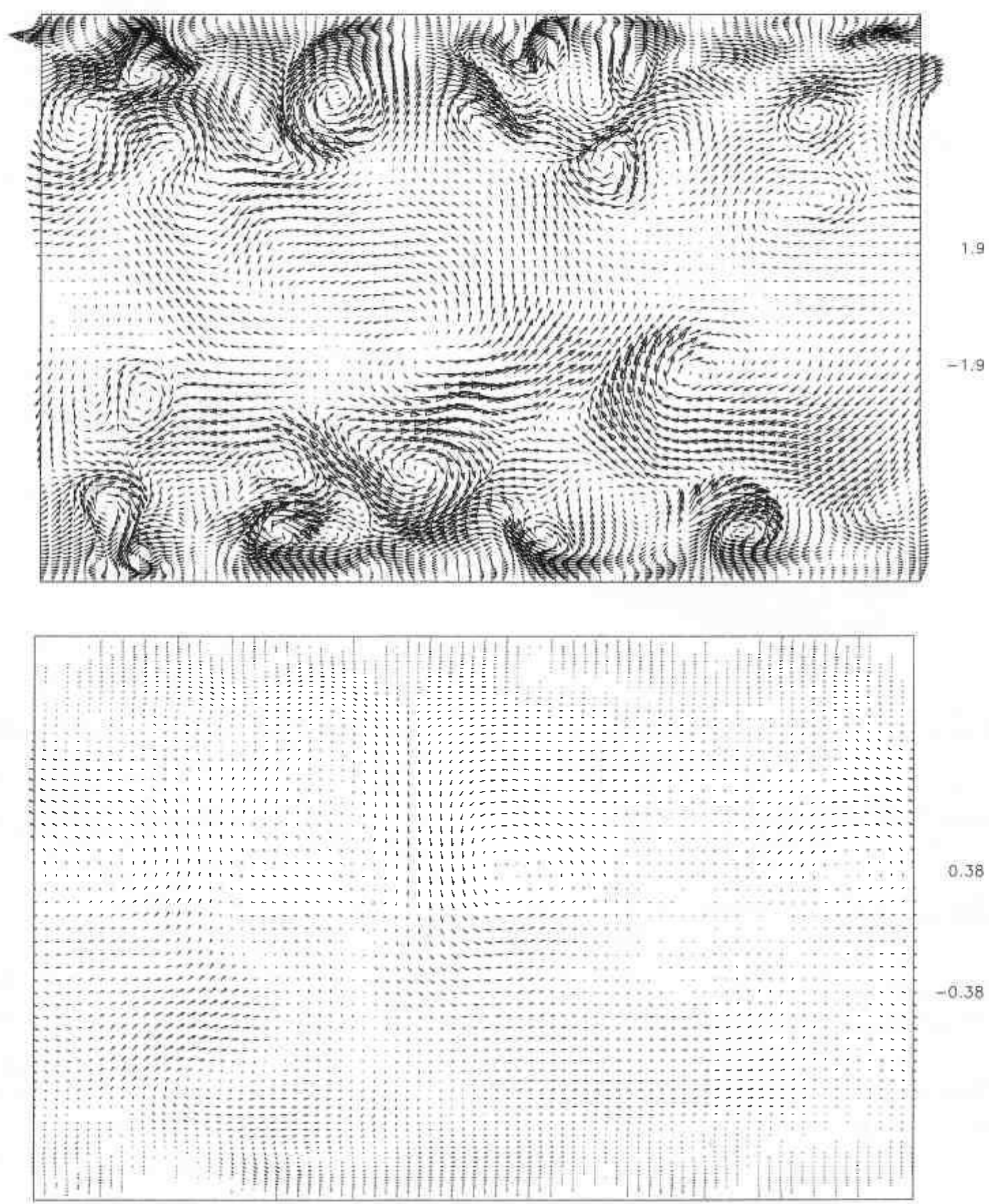

Figure 22. Spanwise vorticity at $t=5000$ for uncontrolled (upper) and controlled (lower) 3D channel flow at $R e=4000$.

Inserting into equation (163), yields

$$
\begin{aligned}
\dot{E}(\mathbf{w}) \leqslant & -2\left(\frac{2}{\operatorname{Re}}-1\right) E(\mathbf{w})+\frac{1}{\operatorname{Re}} \int_{0}^{L} \int_{0}^{2 \pi} v_{r}^{2}(1, \theta, z, t) d \theta d z \\
& -\frac{1}{2} \int_{0}^{L} \int_{0}^{2 \pi} v_{r}^{3}(1, \theta, z, t) d \theta d z-\int_{0}^{L} \int_{0}^{2 \pi} v_{r}(1, \theta, z, t) p(1, \theta, z, t) d \theta d z
\end{aligned}
$$




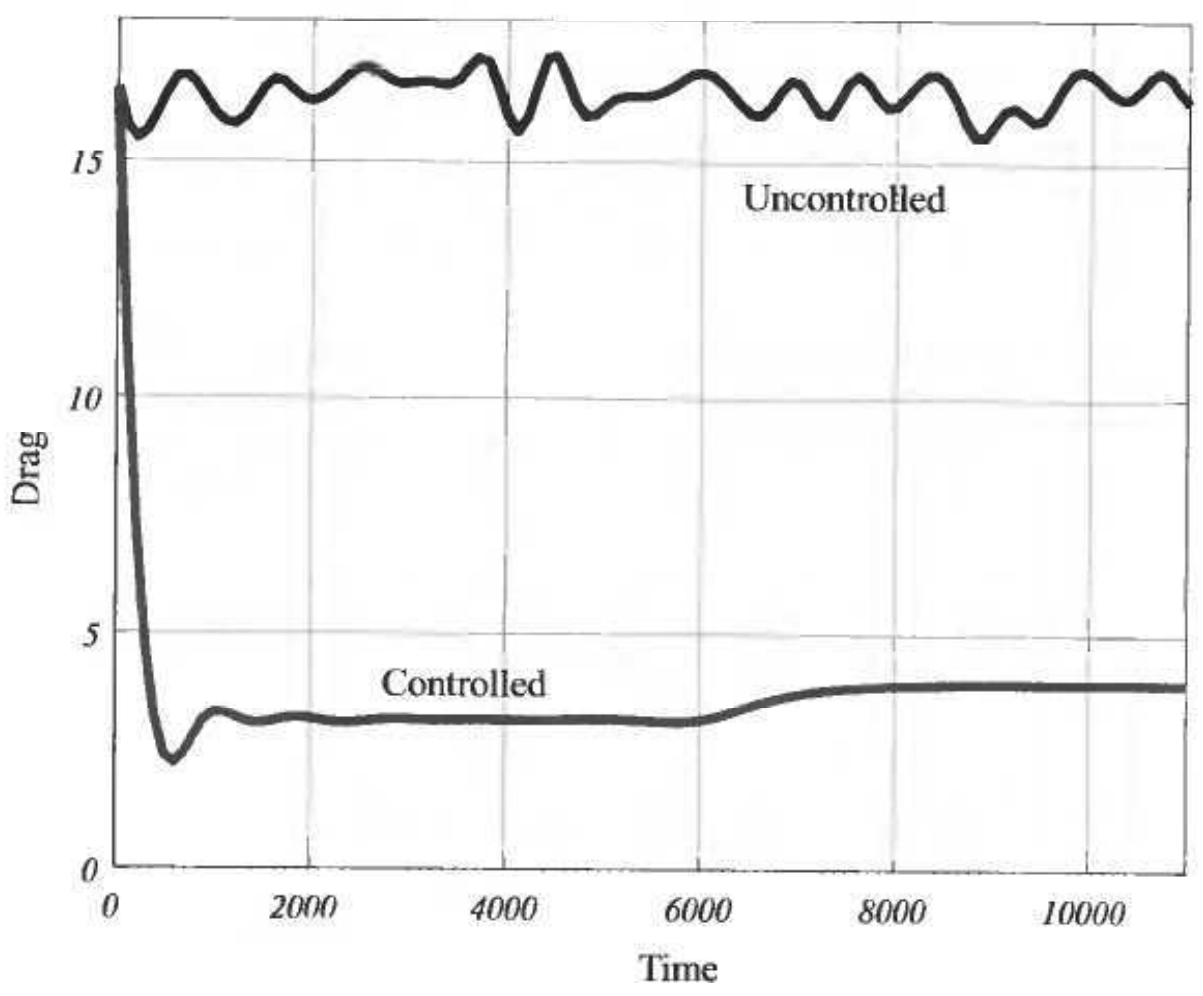

Figure 23. Drag as a function of time for uncontrolled and controlled 3D channel flow at $R e=4000$.

For $n$ odd, we get

$$
\begin{aligned}
\int_{0}^{2 \pi} v_{r}^{n}(1, \theta, z, t) d \theta & =\int_{-\pi}^{0} v_{r}^{n}(1, \theta, z, t) d \theta+\int_{0}^{\pi} v_{r}^{n}(1, \theta, z, t) d \theta \\
& =-\int_{-\pi}^{0} v_{r}^{n}(1,-\theta, z, t) d \theta+\int_{0}^{\pi} v_{r}^{n}(1, \theta, z, t) d \theta
\end{aligned}
$$

and hy a change of variables in the first integral $\left(\theta^{*}=-\theta\right)$, we get

$$
\int_{0}^{2 \pi} v_{r}^{n}(1, \theta, z) d \theta=-\int_{0}^{\pi} v_{r}^{n}\left(1, \theta^{*}, z, t\right) d \theta^{*}+\int_{0}^{\pi} v_{r}^{n}(1, \theta, z, t) d \theta=0
$$

which proves that the net mass flow through the wall is zero, and that

$$
\int_{0}^{2 \pi}\left[v_{r}^{3}\right]_{r=1} d \theta=0
$$

by setting $n=1$ and $n=3$, respectively. Since

$$
\begin{aligned}
\int_{0}^{2 \pi} v_{r}(1, \theta, z, t) p(1, \theta, z, t) d \theta= & \int_{-\pi}^{0} v_{r}(1, \theta, z, t) p(1, \theta, z, t) d \theta \\
& +\int_{0}^{\pi} v_{r}(1, \theta, z, t) p(1, \theta, z, t) d \theta
\end{aligned}
$$




$$
\begin{aligned}
= & -\int_{\pi}^{0} v_{r}\left(1,-\theta^{*}, z, t\right) p\left(1,-\theta^{*}, z, t\right) d \theta^{*} \\
& +\int_{0}^{\pi} v_{r}(1, \theta, z, t) p(1, \theta, z, t) d \theta \\
= & -\int_{0}^{\pi} v_{r}\left(1, \theta^{*}, z, t\right) p\left(1,-\theta^{*}, z, t\right) d \theta^{*} \\
& +\int_{0}^{\pi} v_{r}(1, \theta, z, t) p(1, \theta, z, t) d \theta \\
= & \int_{0}^{\pi} v_{r}(1, \theta, z, t)(p(1, \theta, z, t)-p(1,-\theta, z, t)) d \theta \\
= & \frac{1}{k} \int_{0}^{\pi} v_{r}^{2}(1, \theta, z, t) d \theta=\frac{1}{2 k} \int_{0}^{2 \pi} v_{r}^{2}(1, \theta, z, t) d \theta
\end{aligned}
$$

we obtain

$$
\dot{E}(\mathbf{w}) \leqslant-2\left(\frac{2}{R e}-1\right) E(\mathbf{w})-\left(\frac{1}{2 k}-\frac{1}{R e}\right) \int_{0}^{L} \int_{0}^{2 \pi} v_{r}^{2}(1, \theta, z, t) d \theta d z
$$

Thus, the equilibrium profile is globally exponentially stable in $L_{2}$ for sufficiently small $R e$ and for appropriately selected feedback gain $k$. Note that the control law is decentralized and has a symmetrical structure similar to that of the pressure-based control designed for the 2D channel flow case (see equations (153)-(154)).

\section{Drag Reduction Below Laminar Flow}

\subsection{Wall-normal actuation}

The simple pressure-based feedback control strategy for wall-transpiration control of incompressible unsteady 2D channel flow proposed in Aamo et al. (2001), leads to flow transients with instantaneous drag far lower than that of the corresponding laminar flow (see lower right graph in Figure 17). This touches at the common belief that the laminar flow constitutes a fundamental limit to the drag reduction that is possible to obtain, which is stated in Bewley (2001) as the following conjecture

Conjecture 1 The lowest sustainable drag of an incompressible constant mass-flux channel flow in either $2 \mathrm{D}$ or $3 \mathrm{D}$, when controlled via a distribution of zero-net massflux blowing/suction over the channel walls, is exactly that of the laminar flow.

We denote the drag of the laminar flow $D_{L}$, and by sustainable drag, (denoted $D_{\infty}$ ), we mean the time average (denoted $\bar{D}(t)$ ) of the instataneous drag (denoted $D(t))$ as the averaging time $T$ approaches infinity, i.e.,

$$
D_{\infty} \triangleq \lim _{T \rightarrow \infty} \bar{D}(T) \triangleq \lim _{T \rightarrow \infty} \frac{1}{T} \int_{0}^{T} D(t) d t
$$




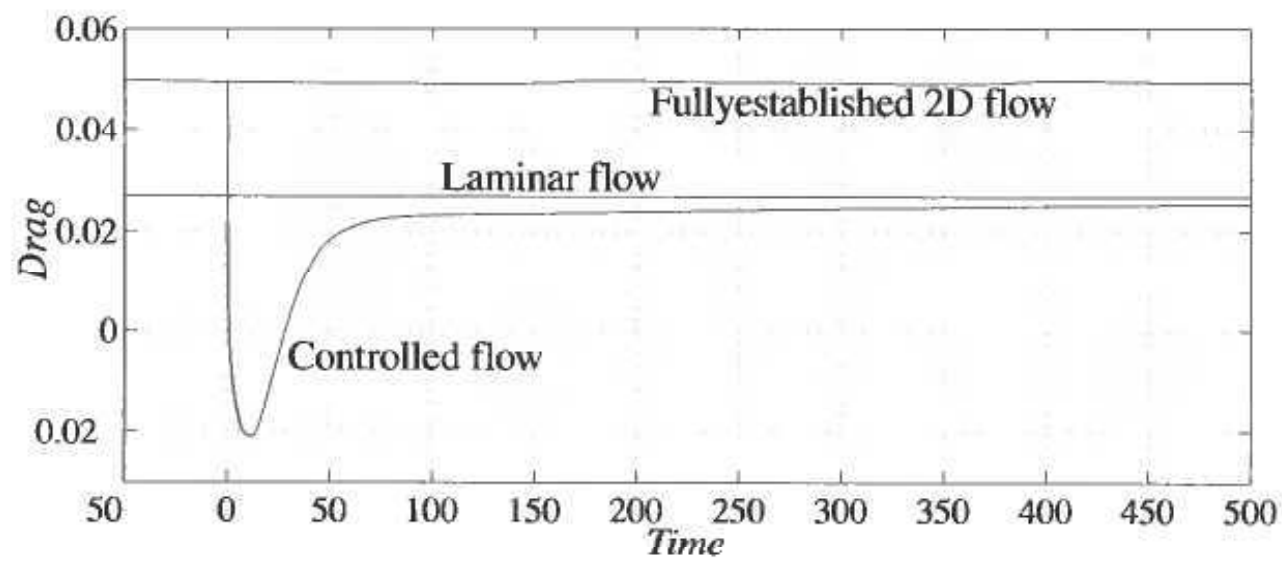

Figure 24. History of drag. Simulation initiated from fully established unsteady 2D flow at $R e=7500$. Stabilizing pressure-based feedback control strategy with $k=-0.125$ turned on at $t=0$.

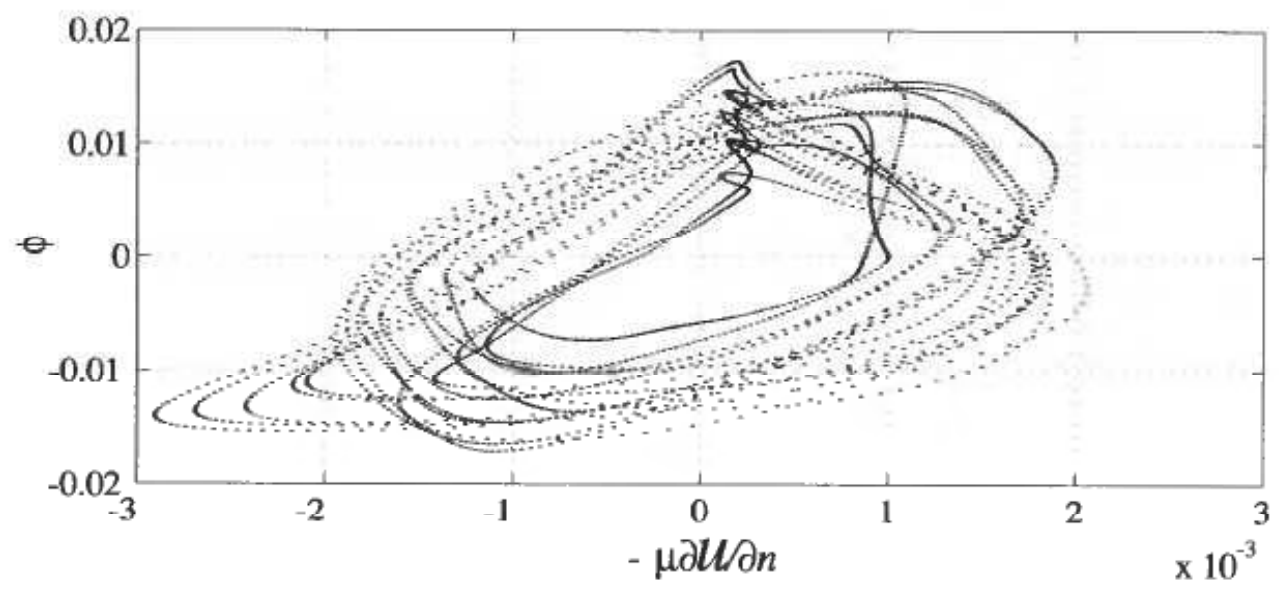

Figure 25. Scatterplot of $\phi$ versus $(-\mu \partial U / \partial n)$ at $t=5$.

with the instantaneous drag given as in equation (161), multiplied by the factor $\mu L$. In Bewley (2001a) the mechanisms that initiate the $D(t)<D_{L}$ transient is investigated, and an attempt at sustaining the drag below that of laminar flow is made. A simulation of a constant mass-flux $2 \mathrm{D}$ channel flow at $R e=7500$, using a box length 60 times the channel half width serves as an illustration. The flow at $t=0^{-}$in Figure 24 , a fully established unsteady flow in a 2D channel (scc, c.g. Jimćncz (1990)), has extensive regions of backflow near the walls. This appears to be the key to initiating a $D(t)<D_{L}$ transient. A scatter plot of the local control $\phi$ as a function of the local value of $(-\mu \partial U / \partial n)$ at $t=5$ (shortly after the control is turned on) is shown in Figure 25, demonstrating correlation of blowing with local regions of positive drag and suction with local regions of negative drag using the present strategy $(76 \%$ of the samples are in the first and third quadrants). By generally applying suction at the walls in regions of negative drag, and applying blowing in regions of large positive drag, the negative drag regions are intensified (locally, more negative drag) and the high positive drag regions are moderated (locally, less positive drag), as illustrated in Figure 26. In terms of reducing the total instantaneous drag $D(t)$ integrated over the walls at time $t=5$, both effects are beneficial, and thus the control application results, 


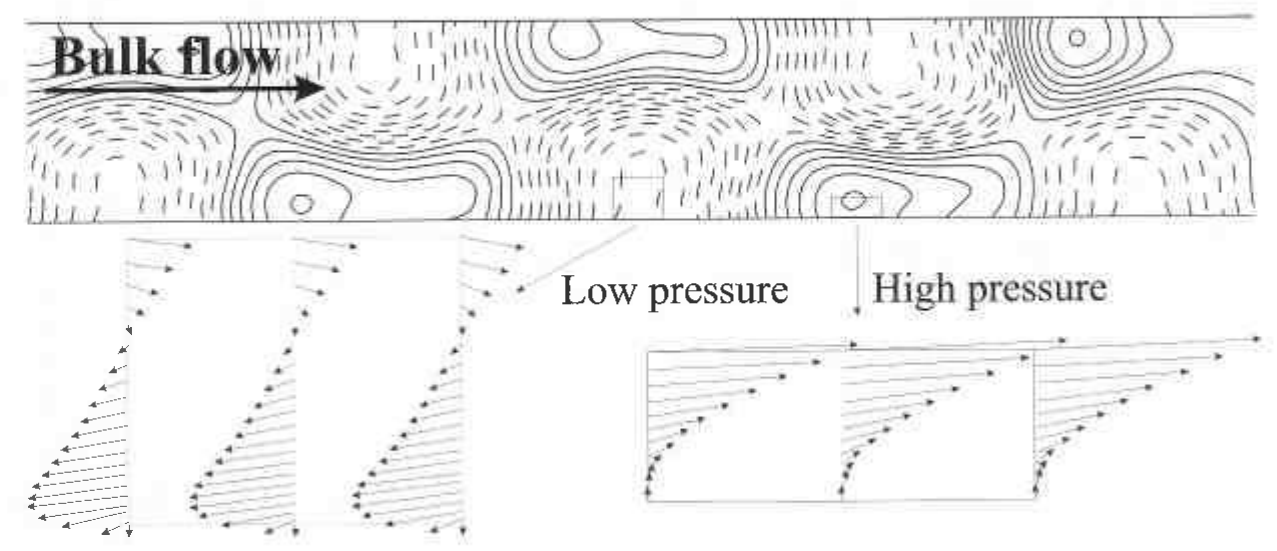

Figure 26. Win-Win mechanism at $t=5$ : intensification of local regions of negative drag by suction in low pressure regions and moderation of positive drag by blowing in high pressure regions. Shown are contours of pressure in 1/6 of the computational domain (top) and selected velocity profiles (bottom).

for a brief amount of time, in a 'win-win' situation, facilitating a drastic transient reduction in skin-friction drag to well below laminar levels. Unfortunately, the wall suction quickly acts to remove the backflow from the flow domain entirely, after which the instantaneous drag $D(t)$ asymptotes back to the laminar level, $D_{L}$.

A metric which quantifies the degree of backflow present at any instant in a particular flow is given by

$$
b_{p}=\left[\frac{1}{A} \int_{\Omega^{-}}|U|^{p} d \Omega\right]^{1 / p}
$$

where $\Omega^{-}$is the subset of the channel flow domain $\Omega$ which is characterized by regions of flow with negative streamwise velocity, i.e. $\Omega^{-}=\{\Omega(x, y) \mid U(x, y)<0\}$, and $A$ is the volume of the entire channel domain $\Omega$. For the simulation depicted in Figures 24-26, plots of the history of $b_{1}$ and $b_{2}$ are shown in Figure 27. Note that, by both measures, the backflow is quickly eliminated after the control is initiated; flow visualizations such as Figure 26 demonstrate clearly that the backflowing fluid in $\Omega^{-}$is simply removed from the channel by the control suction.

As a 'standard' problem to test the utility of a given control strategy for reducing

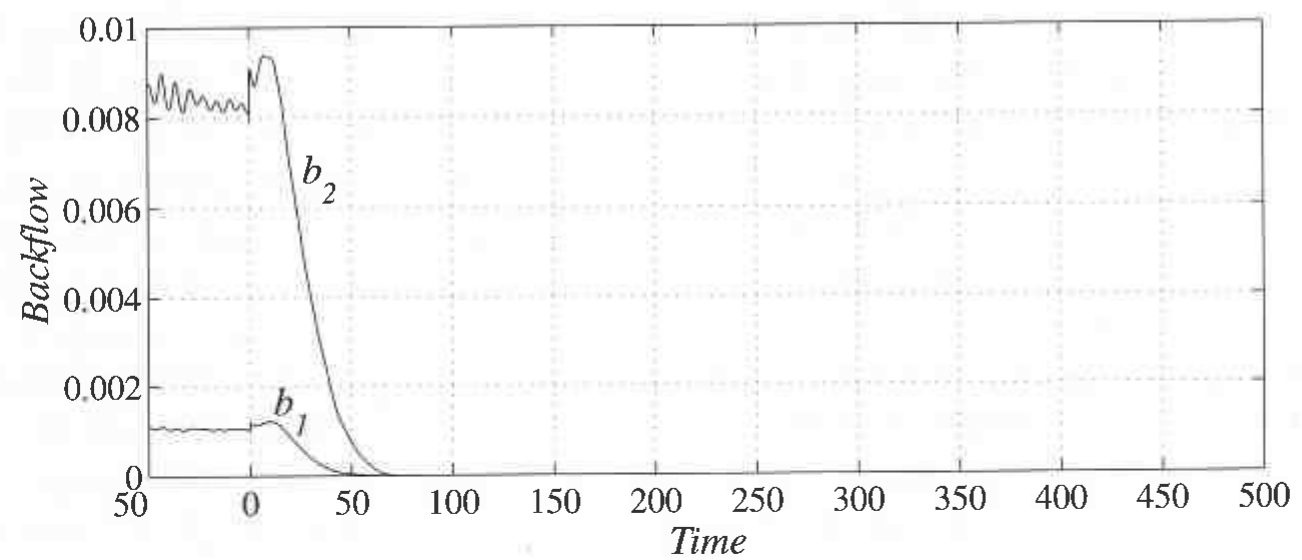

Figure 27. Elimination of backflow after control is turned on, as measured by $b_{1}(t)$ and $b_{2}(t)$. 
Table 1. Forcing schedules explored during parametric study: $T_{\text {cycle }}$ indicates the periud of the cycle used, $T_{1}$ denotes the duration of the first segment of the cycle, $T_{2}$ denotes the duration of the second segment, $k_{1}$ denotes the feedback coefficient used during the first segment, and $k_{2}$ denotes the feedback coefficient during the second segment

\begin{tabular}{lccccc}
\hline Case & $T_{\text {cycle }}$ & $T_{1}$ & $T_{2}$ & $k_{1}$ & $k_{2}$ \\
\hline 1 & 3000 & 2600 & 400 & 0 & 0.125 \\
2 & 3000 & 2700 & 300 & 0 & 0.125 \\
3 & 3000 & 2800 & 200 & 0 & 0.125 \\
4 & 3000 & 2900 & 100 & 0 & 0.125 \\
5 & 3000 & 2950 & 50 & 0 & 0.125 \\
6 & 3000 & 2000 & 1000 & 0 & 0.031 \\
7 & 3000 & 2500 & 500 & 0 & 0.031 \\
8 & 3000 & 2800 & 200 & 0 & 0.031 \\
9 & 2000 & 1600 & 400 & 0 & 0.125 \\
10 & 2000 & 1700 & 300 & 0 & 0.125 \\
11 & 2000 & 1800 & 200 & 0 & 0.125 \\
12 & 2000 & 1900 & 100 & 0 & 0.125 \\
13 & 2000 & 1950 & 50 & 0 & 0.125 \\
14 & 1000 & 250 & 750 & -0.031 & 0.031 \\
15 & 1000 & 350 & 650 & -0.031 & 0.031 \\
16 & 1000 & 500 & 500 & -0.031 & 0.031 \\
\hline
\end{tabular}

time-averaged drag to below laminar levels, a series of controlled 2D channel flow simulations at $R e=7500$ were initialized from small (random) perturbations to a laminar flow profile. The control producing the $D(t)<D_{L}$ transients was cycled off and on periodically, with the 'running average' of the drag, $\bar{D}(t)=\int_{0}^{t} D(t) d t^{\prime}$, computed as the flow evolved to quantify progress towards sustained drag reduction. A large variety of different periods, duty cycles, and control amplitudes were explored; Table I summarizes specific cases examined in detail.

Cases 1-5 reported in Table 1 were executed at a cycle time of $T_{\text {cycle }}=3000$ for a variety of duty cycles with relatively strong stabilizing feedback applied during the second segment of each cycle. Cases 6-8 were similar, but applied relatively weak stabilizing feedback. Cases 9-13 returned to the relatively strong stabilizing feedback, but investigated a shorter cycle time. Finally, cases 14-16 were execuled with destabilizing feedback applied during the first segment of each cycle, and stabilizing feedback applied during the second segment of each cycle; this was done to accelerate the formation of the backflow regions. Histories of the $L_{2}$ energy, the instantaneous and 'running time-averaged' drag $D(t)$ and $\bar{D}(t)$, and the backflow measures $b_{1}$ and $b_{2}$ are illustrated in Figure 28 for four representative cases.

It was found in cases $1,2,9,10$, and 14 , with $T_{2}$ relatively large, that the stabilization provided by the control during the second segment of each cycle was sufficient to stabilize the cntire channel flow back to the parabolic profile; to illustrate, case 14 is plotted in Figure 28(c). These cases imply that $T_{2}$ must be a sufficiently small fraction of $T_{\text {cycle }}$ in order to allow a quasi-periodic behavior to establish.

It was found in cases $5,8,13$, and 16 , with $T_{2}$ relatively small, that the uncontrolled (or, in case 16, destabilized) evolution of the flow during the first segment of each cycle was sufficient to drive the time-averaged drag to heightened levels.

A tradeoff is thus identified: decrease $T_{2}$ and there will be more backflow to exploit during each cycle (so the transient will be more effective at reducing drag), but by allowing the 2D unsteady flow to evolve for a longer time uncontrolled or 
(a)
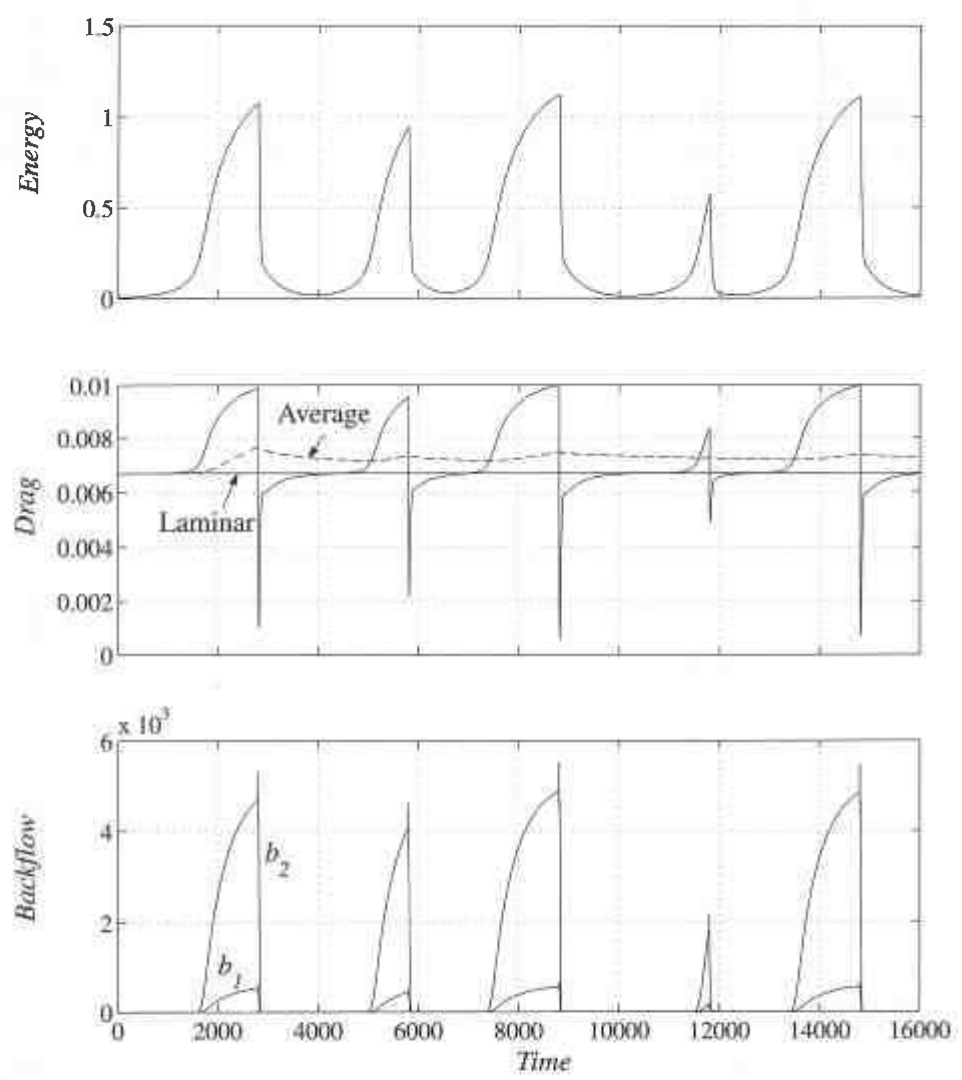

(b)
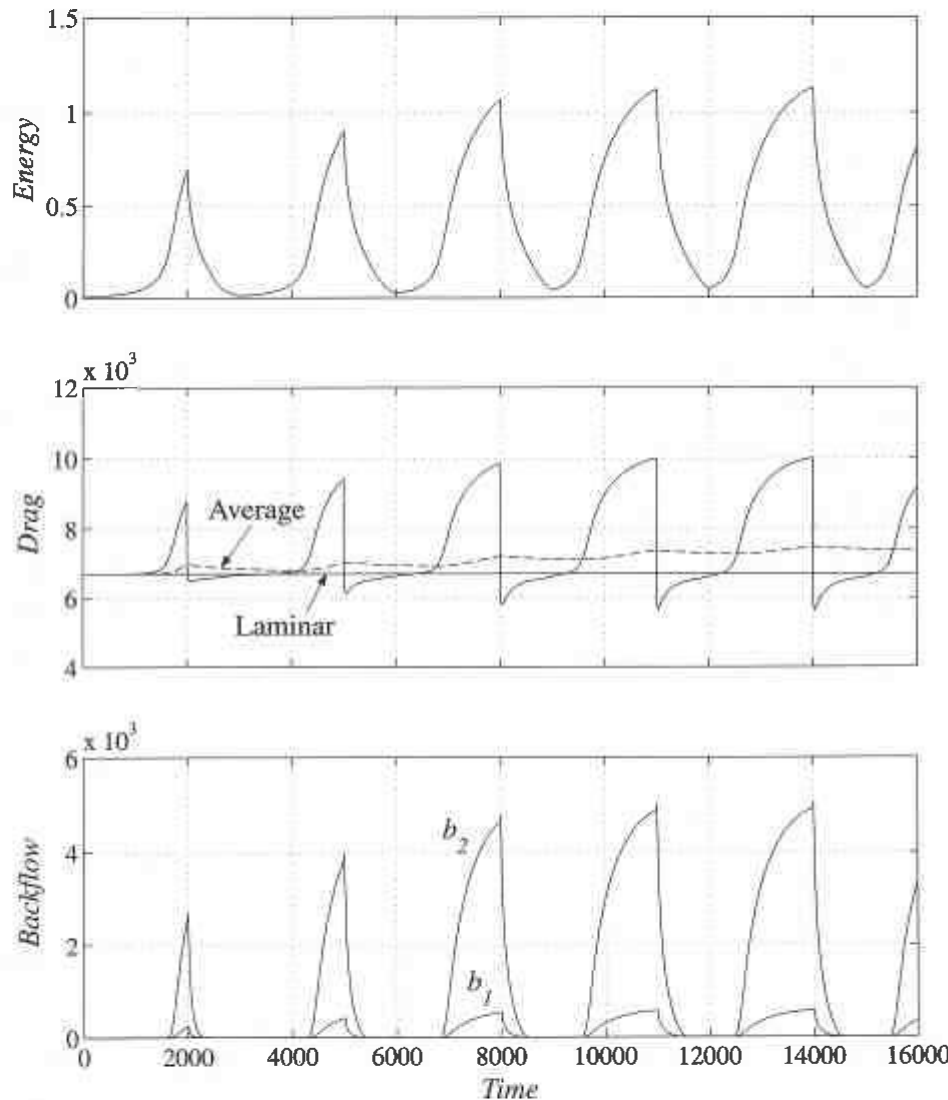

Figure 28. (a) Case 3: $T_{\text {cycle }}=3000$, no feedback for $T_{1}=2600$, relatively strong stabilizing feedback for $T_{2}=400$; (b) Case 6: $T_{\text {cycle }}=3000$, no feedback for $T_{1}=2000$, relatively weak stabilizing feedback for $T_{2}=1000$. 
(c)
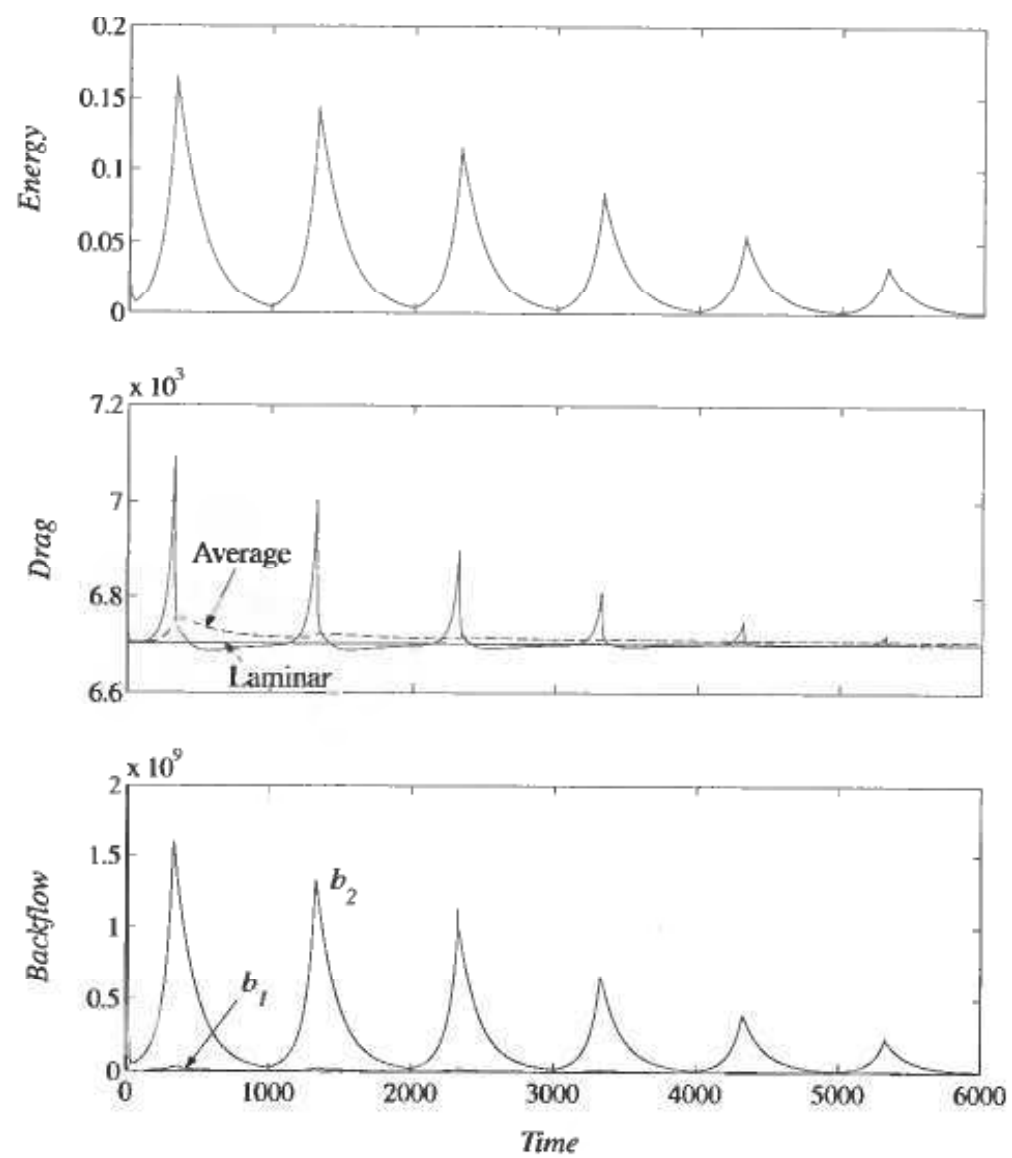

(d)
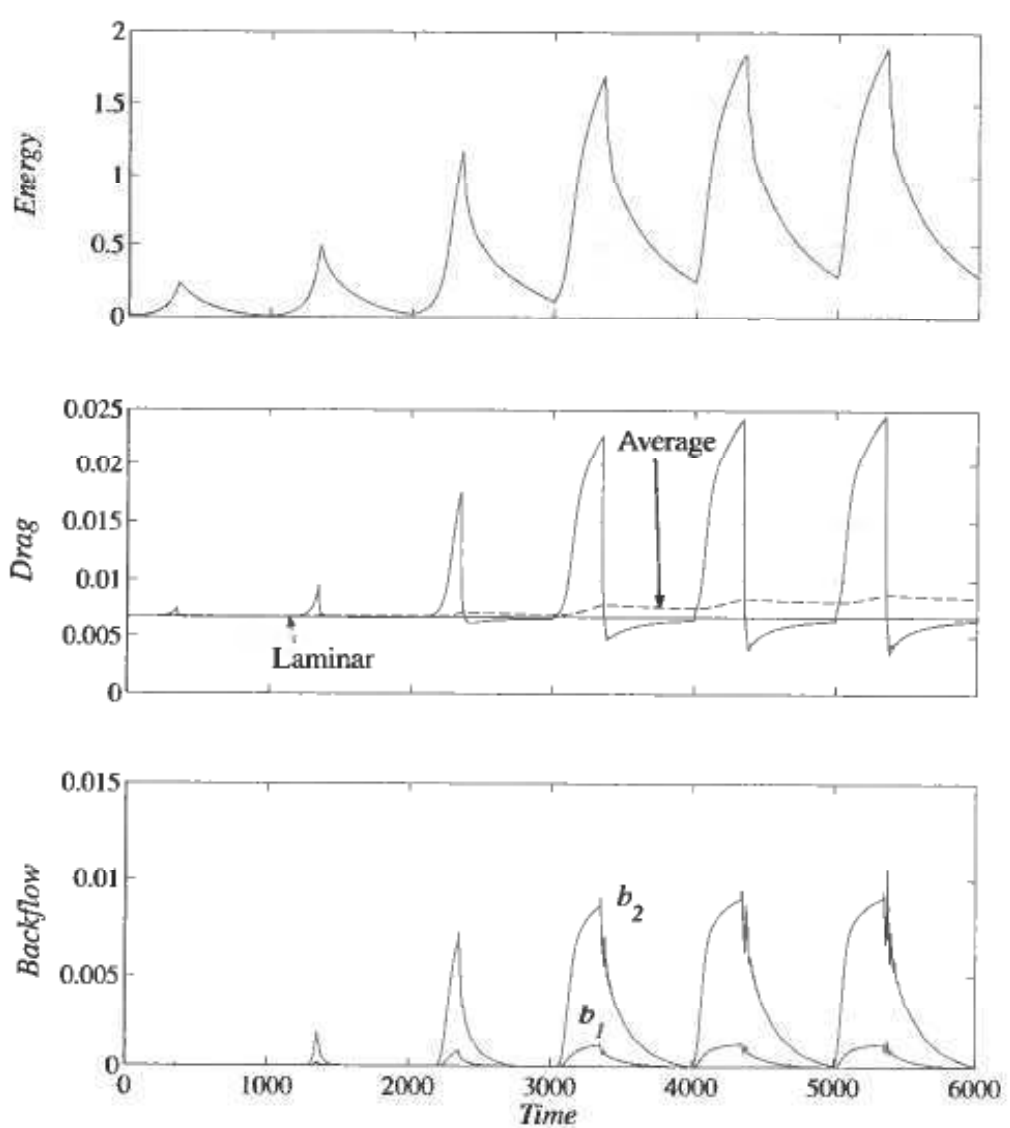

Figure 28. (c) Case 14: $T_{\text {cycle }}=1000$, desabilizing feedback for $T_{1}=325$, stabilizing feedback for $T_{2}=675$; (d) Case 15: $T_{\text {cycle }}=1000$, destabilizing feedback for $T_{1}=350$, stabilizing feedback for $T_{2}=650$. 
destabilized, the mean drag is pulled up higher above the laminar level. Intermediate values of $T_{2}$ were sought for a variety of cycle times and forcing amplitudes over a parametric study of several simulations, some of which are reported here. Over all these simulations, this tradeoff was evident, and not once did the running average, $\bar{D}(t)$, dip below the laminar value when the simulations were initiated from the perturbed laminar state. These results indicate that it is always necessary to pay a more expensive price (in terms of the time-averaged drag) to obtain the backflow than the benefit (in terms of the time-averaged drag) that can be obtained by applying suction to the backflow regions. Therefore, it appears that the parabolic equilibrium profile represents a fundamental limit to the drag reduction possible when applying control based on wall transpiration with zero-net mass flow.

\subsection{Wall-tangential actuation}

The objective of the control strategies above has been to stabilize the parabolic equilibrium profile. Although not explicitly stated in the objective, drag reduction is obtained indirectly using this strategy. For wall normal actuation, with zero-net mass flow, the objective of stabilizing the parabolic profile is equivalent to minimizing drag, due to the conjecture stated in Section 7.1. However, it is clear that drag can be reduced further if actuation is directed in the streamwise direction, and in particular if it is allowed a steady-state offset. This calls for new actuators that allow slip boundary conditions at rigid walls. Advancements in this direction based on conveyer belts as actuators, allowing slip boundary conditions, are currently being persued (Bewley, 2001b).

\section{Concluding Remarks}

The field of flow control has picked up pace over the past decade or so, on the promise of real-time distributed control on turbulent scales being realizable in the near future. This promise is due to the micromachining technology that emerged in the 1980s, and developed at an amazing speed through the 1990s. In lab experiments, so called micro-electro-mechanical systems (MEMS) that incorporate the entire detection-decision-actuation process on a single chip, have been batch processed in large numbers and assembled into flexible skins for gluing onto body-fluid interfaces for drag reduction purposes.

Control of fluid flows span a wide variety of specialities. In Part I of this tutorial, we have focused on the problem of reducing drag in channel and pipe flows by stabilizing the parabolic equilibrium profile using boundary feedback control. The control strategies used for this problem include classical control, based on the Nyquist criteria, and various optimal control techniques $\left(\mathscr{H}_{2}, \mathscr{H}_{\infty}\right)$, as well as applications of Lyapunov stability theory. The linear approaches are clearly restrictive in that they only provide local stability results. The nonlinear approaches give global stability results, but impose serious restrictions on the Reynolds number. Thus, the problem of stabilizing the parabolic equilibrium profile of channel and pipe flows in general, remains open. Nevertheless, stabilization is achieved for large Reynolds numbers in simulations.

\section{Acknowledgements}

The authors would like to thank Dr. Andras Balogh (UCSD) and Professor Thomas Bewley (UCSD) for permitting reproduction of selected figures and pictures 
from their work, and Dr. Svein Ivar Sagatun (Norsk Hydro) for valuable comments during the preparation of this tutorial. The support from Norsk Hydro and the Norwegian Research Council is gratefully acknowledged.

\section{REFERENCES}

Aamo, O. M., Krstić, M. and Bewley, T. R. (2002). Control of Mixing by Boundary Feedback in 2D Channel Flow. Submitted.

Abergel, F. and Temam, R. (1990). On some control problems in fluid mechanics. Theoret. and Comput. Fluid Dynamics, 1, 303-325.

Baker, J., Armaou, A. and Christofides, P. D. (2000). Drag Reduction in Incompressible Channel Flow Using Electromagnetic Forcing. In Proceedings of the American Control Conference, Chicago, Illinois, USA.

Balogh, A., LiU, W-J. and Krstić, M.(1999). Stability Enhancement by Boundary Control in 2D Channel Flow-Part I: Regularity of Solutions. In Proceedings of the 38th Conference on Decision and Control, Phoenix, Arizona, USA.

Balogh, A., Liu, W-J. and KrSTić, M. (2001). Stability Enhancement by Boundary Control in 2D Channel Flow. Accepted subject to revision for IEEE Transactions on Automatic Control.

BALOGH, A. (2002) On stabilization of 3D channel flow. Private communication.

Bamieh, B., Paganini, F. and Dahleh, M. (2000). Distributed Control of Spatially-Invariant Systems. Preprint.

Barbu, V. (1997). The time optimal control of Navier-Stokes equations. Systems \& Control Letters, 30, 93-100.

Barbu, V. and Skitharan, S. S. (1998). $H^{\infty}$-control theory of fluid dynamics. $R$. Soc. Lond. Proc. Ser. A Math. Phys. Eng. Sci., 454(1979), 3009-3033.

BewLeY, T. R. and LrU, S. (1998). Optimal and robust control and estimation of linear paths to transition. Journal of Fluid Mechanics, 365, 305.349.

Bewley, T. R. (2001). Flow Control: New Challenges for a New Renaissance. Progress in Aerospace Sciences, 37, 21-58.

Bewley, T. R. and Moin, P. (1999). Technical report TF-76, Stanford Iniversity.

Bewley, T. R., MoIN, P. and TEMAM, R. (2000). DNS-based predictive control of turbulence: an optimal benchmark for feedback algorithms. Preprint.

Bewley, T. R., Temam, R. and Ziane, M. (2000). A general framework for robust control in fluid mechanics. Physica D, 138, 360-392.

BEWLEY, T. R. and AAMO, O. M. (2002). On the search for fundamental performance limitations in fluid-mechanical systems, Proceedings of the 2002 ASME Fluids Engineering Division Summer Meeting, Montreal, Quebec, Canada.

BEWLEY, T. R. (2002). Analysis of a versatile moving-belt mechanism for the control of wallbounded flows. In preparation.

Bird, R. B., Stewart, W. E. and Lightfoot, E. N. (1960). Transport Phenomena. John Wiley \& Sons, Inc.

ButLeR, K. M. and FARRELL, B. F. (1992). Three-dimensional optimal perturbations in viscous shear flows. Physics of Fluids A, 4, 16371650.

Canuto, C. Hussaini, M. Y., Quarteroni, A. and Zang, T. A. (1988). Spectral Methods in Fluid Dynamics. Springer-Verlag.

Christofides, P. D. and Armaou, A. (1998). Nonlinear Control of Navier-Stokes Equations. In Proceedings of the American Control Conference, Philadelphia, Pennsylvania.

CORON, J.-M. (1996). On the controllability of the 2-D incompressible Navier-Stokes equations with the Navier slip boundary conditions. ESAIM: Control, Optim. Cal. Var., 1, 35-75.

CORON, J.-M. (1998). On null asymptotic stabilization of the 2-D Euler equation of incompressible fluids on simply connected domains. Prepublications 98-59 Université de Paris-Sud, Mathematiques.

CortelezzI, L., Lee, K. H., KIM, J. and SPeyer, J. L. (1998). Skin-friction drag reduction via robust reduced-order linear feedback control. Int. J. Comput. Fluid Dyn., 8(1-2), 79-92.

CORTELEZZI, L. and SPEYER, J. L. (1998). Robust reduced-order controller of laminar boundary layer transitions. Physical Review E, 58(2). 
FATTORINI, H. O. and SRITHARAN, S. S. (1998). Optimal control problems with state constraints in fluid mechanics and combustion. Appl. Math. Optim., 38(2), 159-192.

Fernández-CARA, E. and GonzÁlez-Burgos, M. (1995). A result concerning controllability for the Navier-Stokes equations. SIAM J. Control Optim., 33(4), 1061-1070.

Ferziger, J. H. and Perić, M. (1999). Computational Methods for Fluid Dynamics. SpringerVerlag.

Fursikov, A. V., Gunzburger, M. D. and Hou, L. S. (1998). Boundary value problems and optimal boundary control for the Navier-Stokes system: the two-dimensional case. SIAM J. Control Optim., 36, 852-894.

Gad-EL-HaK, M. (1996). Modern developments in flow control. Appl. Mech. Rev., 49(7), 365-379.

GAD-EL-HAK, M. (2000). Flow Control: Passive, Active and Reactive Flow Management. Cambridge University Press.

Gunzburger, M. D., Hou, L. and Svobodny, T. P. (1990). A numerical method for drag minimization via the suction and injection of mass through the boundary. In Stabilization of Flexible Structures (ed. J. P. ZoLEsro), Springer-Verlag.

Gunzburger, M. D. (Editor) (1995). Flow Control. Springer-Verlag.

Ho, C-M. and TAI, Y-C. (1996). REVIEW: MEMS and its applications for flow control. Journal of Fluids Engineering, 118, 437-447.

Ho, C-M. and TAI, Y-C. (1998). Micro-electro-mechanical systems (MEMS) and fluid flows. Annu. Rev. Fluid Mech., 30, 579-612.

Hou, L. S. and YAN, Y. (1997). Dynamics for controlled Navier-Stokes systems with distributed controls. SIAM J. Control Optim., 35(2), 654-677.

HögBeRG, M. and BewLEY, T. R. (2000). Spatially-compact convolution kernels for decentralized control and estimation of transition in plane channel flow. Preprint.

ImanUVILOV, O. Y. (1998). On exact controllability for the Navier-Stokes equations. ESAIM: Control, Optim. Cal. Var., 3, 97-131.

Iто, K. and KANG, S. (1994). A dissipative feedback control synthesis for systems arising in fluid dynamics. SIAM J. Control Optim., 32(3), 831-854.

JiMÉNEZ, J. (1990). Transition to turbulence in two-dimensional Poiseuille flow. Journal of Fluid Mechanics, 218, 265-297.

Joshi, S. S., SPeYer, J. L. and Kim, J. (1997). A systems theory approach to the feedback stabilization of infinitesimal and finite-amplitude disturbances in plane Poiseuille flow. Journal of Fluid Mechanics, 332, 157-184.

Joshi, S. S., Speyer, J. L. and KIM, J. (1999). Finite Dimensional Optimal Control of Poiseuille Flow. Journal of Guidance, Control, and Dynamics, 22(2), 340-348.

JuRdJEvic, V. and QuINN, J. P. (1978). Controllability and Stability. Journal of Differential Equations, 28, 381-389.

Kang, S. M., Ryder, V., Cortelezzi, L. and Speyer, J. L. (1999). State-Space Formulation and Controller Design for Three-Dimensional Channel Flows. In Proceedings of the American Control Conference, San Diego, California.

Krstić, M. (1999). On global stabilization of Burgers' equation by boundary control. Systems \& Control Letters, 37, 123-141.

Lagnese, J. E., Russell, D. L. and White, L. (Editors) (1995). Control and Optimal Design of Distributed Parameter Systems. Springer-Verlag.

RozhdestVensky, B. L and SimAKIN, I. N. (1984). Secondary flows in a plane channel: their relationship and comparison with turbulent flows. Journal of Fluid Mechanics, 147, 261-289.

SRITHARAN, S. S. (1991). Dynamic programming of the Navier-Stokes equations. Systems and Control Letters, 16, 299-307.

SRITHARAN, S. S. (1998). Optimal control of viscous flows. SIAM. 